\title{
Chemistry in infrared dark clouds ${ }^{\star}$
}

\author{
T. Vasyunina ${ }^{1, \star \star}$, H. Linz ${ }^{1}$, Th. Henning ${ }^{1}$, I. Zinchenko ${ }^{2}$, H. Beuther ${ }^{1}$, and M. Voronkov ${ }^{3}$
}

1 Max Planck Institute for Astronomy (MPIA), Königstuhl 17, 69117 Heidelberg, Germany

e-mail: [vasyunina, linz, henning, beuther] @mpia.de

2 Institute of Applied Physics of the Russian Academy of Sciences, Ulyanova 46, 603950 Nizhny Novgorod, Russia e-mail: zin@appl.sci-nnov.ru

3 Australia Telescope National Facility, CSIRO Astronomy and Space Science, PO Box 76, Epping, NSW 1710, Australia e-mail: maxim. voronkov@csiro.au

Received 11 May 2010 / Accepted 3 December 2010

\section{ABSTRACT}

Context. Massive stars play an important role in shaping the structure of galaxies. Infrared dark clouds (IRDCs), with their low temperatures and high densities, have been identified as the potential birthplaces of massive stars. In order to understand the formation processes of massive stars, the physical and chemical conditions in infrared dark clouds have to be characterized.

Aims. The goal of this paper is to investigate the chemical composition of a sample of southern infrared dark clouds. One important aspect of the observations is to check, whether the molecular abundances in IRDCs are similar to the low-mass pre-stellar cores, or if they show signatures of more evolved evolutionary stages.

Methods. We performed observations toward 15 IRDCs in the frequency range between 86 and $93 \mathrm{GHz}$ using the 22-m Mopra radio telescope. In total, 13 molecular species comprising $\mathrm{N}_{2} \mathrm{H}^{+},{ }^{13} \mathrm{CS}, \mathrm{CH}_{3} \mathrm{CN}, \mathrm{HC}_{3} \mathrm{~N}, \mathrm{HNC}, \mathrm{HCO}^{+}, \mathrm{HCN}, \mathrm{HNCO}, \mathrm{C}_{2} \mathrm{H}, \mathrm{SiO}, \mathrm{H}^{13} \mathrm{CO}^{+}$, $\mathrm{H}^{13} \mathrm{CN}$, and $\mathrm{CH}_{3} \mathrm{C}_{2} \mathrm{H}$ were observed for all targets. Hence, we included in general species appropriate for elevated densities, where some of them trace the more quiescent gas, while others are sensitive to more dynamical processes.

Results. We detect $\mathrm{HNC}, \mathrm{HCO}^{+}$, and $\mathrm{HNC}$ emission in all clouds and $\mathrm{N}_{2} \mathrm{H}^{+}$in all IRDCs except one. In some clouds we detect $\mathrm{SiO}$ emission. Complicated shapes of the $\mathrm{HCO}^{+}$emission line profile are found in all IRDCs. Both signatures indicate infall and outflow motions and the beginning of star-formation activity, at least in some parts of the IRDCs. Where possible, we calculate molecular abundances and make a comparison with previously obtained values for low-mass pre-stellar cores and high-mass protostellar objects (HMPOs). We show a tendency for IRDCs to have molecular abundances similar to low-mass pre-stellar cores rather than to HMPOs abundances on the scale of our single-dish observations.

Key words. ISM: clouds - ISM: molecules - radio lines: ISM - stars: formation

\section{Introduction}

Massive stars play an important role in determining physical, chemical, and morphological properties of galaxies. The last decade has seen considerable progress in the understanding of massive star-formation (Zinnecker \& Yorke 2007). Since their detection by Perault et al. (1996) and Egan et al. (1998), cold and dense infrared dark clouds (IRDCs) appear to be the ideal sites to investigate the initial conditions for the process of massive starformation. Infrared dark clouds have typical sizes between 1 and $10 \mathrm{pc}$, masses from several hundreds to several thousands solar masses and $\mathrm{H}_{2}$ column densities between 2 and $10 \times 10^{23} \mathrm{~cm}^{-2}$ (e.g. Rathborne et al. 2006; Vasyunina et al. 2009; Ragan et al. 2009).

Apart from continuum observations, molecular line data have been used to characterize the properties of IRDCs. The first molecular line data were obtained by Carey et al. (1998), who detected $\mathrm{H}_{2} \mathrm{CO}$ in 10 clouds, thus confirming the presence of dense gas. Using a large velocity gradient (LVG) model they estimated $\mathrm{H}_{2} \mathrm{CO}$ abundances of $\sim 10^{-10}$. That is a factor of 50 lower compared with low-density clouds and can be explained by accretion of gas-phase metals onto dust grains in the cold and

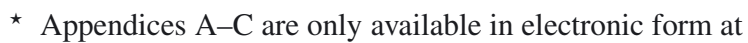
http: //www . aanda.org

$\star \star$ Member of the International Max Planck Research School (IMPRS) Heidelberg.
}

dense IRDCs. Ammonia observations by Sridharan et al. (2005) and Pillai et al. (2006) allowed a temperature determination of IRDCs in a range from 10 to $20 \mathrm{~K}$.

While earlier studies (Simon et al. 2006) suggested the association of most IRDCs with the so-called Galactic molecular ring (galactocentric distance of $5 \mathrm{kpc}$ ), the Jackson et al. (2008) study gave new evidence that the IRDC distribution in the first and fourth galactic quadrant more closely follows a galactic spiral arm (the Scutum-Centaurus arm). Because in normal spiral galaxies OB stars seem to form primarily in spiral arms, the association of IRDCs with a Milky Way spiral arm supports the idea that IRDCs are related to high-mass star formation.

Sakai et al. (2008) observed $\mathrm{N}_{2} \mathrm{H}^{+}(1-0), \mathrm{HC}_{3} \mathrm{~N}(5-4)$, $\mathrm{CCS}\left(4_{3}-3_{2}\right), \mathrm{NH}_{3}(1,1),(2,2),(3,3)$, and $\mathrm{CH}_{3} \mathrm{OH}(7-6)$ lines toward the massive clumps associated with IRDCs to study the chemical conditions in IRDCs. Analyzing the CCS and $\mathrm{N}_{2} \mathrm{H}^{+}$ abundance ratio, they conclude that infrared dark clouds are chemicaly more evolved than low-mass pre-stellar cores.

An estimate of the chemical evolutionary status of IRDCs was performed also by Gibson et al. (2009). Using $\mathrm{C}^{18} \mathrm{O}, \mathrm{CS}$, and $\mathrm{N}_{2} \mathrm{H}^{+}$abundances and a chemical evolution code, they showed that cores where all three lines are detected appear to be chemically young $\left(10^{4.5}<t<10^{5.5}\right.$ years $)$. Cores where no $\mathrm{N}_{2} \mathrm{H}^{+}$emission is detected are suspected to be especially young $\left(t<10^{2}\right.$ years). This suggests that these regions may not yet have formed massive protostars. 
A molecular survey by Sakai et al. (2010) toward 20 massive clumps including mid-IR bright and mid-IR dark sources showed outflow activities and the possible presence of protostars in some clouds. Their line width analysis allowed the authors to reconstruct the distribution of the molecular species at the different evolutionary stages of massive clumps.

While many characteristics of infrared dark clouds were determined during the last ten years, some of their properties are still not well known. Among the open questions is the chemical state of these clouds. What is the chemistry in IRDCs? Is it really different from the chemistry in low-mass dark clouds?

To enlarge the sample of well characterized IRDCs, we started a program to study gas and dust properties of southern infrared dark clouds (Vasyunina et al. 2009, Paper I hereafter). A set of 15 clouds was selected in the pre-Spitzer era by visual examination of the extinction contrast of the MSX $8.3 \mu \mathrm{m}$ images. In the meantime, the Spitzer satellite has succeeded MSX and provided a far higher spatial resolution and sensitivity. GLIMPSE mid-infrared images of our regions were retrieved from the Spitzer Archive. Continuum 1.2 mm maps were obtained with the SIMBA bolometer array at the SEST telescope. A $1.2 \mathrm{~mm}$ and $8 \mu \mathrm{m}$ study of these southern IRDCs showed that these objects are not just distant Taurus-like clouds, but a distinct type of clouds with a clear trend to higher $\mathrm{H}_{2}$ column densities. It was found that the true peak column densities, extracted from millimeter data, exceed $3 \times 10^{23} \mathrm{~cm}^{-2}$ ( or $1 \mathrm{~g} \mathrm{~cm}^{-2}$ ), which has been proposed as a threshold for high-mass star-forming clouds (Krumholz \& McKee 2008).

This paper is our next step toward the understanding of the nature of the IRDCs. Here we present our investigations of the chemical composition of southern clouds. We perform molecular line observations in the $3 \mathrm{~mm}$ band with the Mopra singledish radio telescope. Combining molecular line data and $\mathrm{H}_{2}$ column densities from the previous study we estimate molecular abundances and compare them with results for low-mass prestellar cores. The analysis of molecular lines provides not only information about chemistry, but also about physical processes in molecular clouds. For instance, the presence of $\mathrm{SiO}$ emission and $\mathrm{HCO}^{+}$extended wings is evidence for outflow activity in a cloud. Specific line shapes can indicate infall motion. Detection or non-detection of some species helps to determine the evolutionary status of our targets.

The paper is organized in the following way. In Sect. 2 we describe our target selection, the selected molecular lines, observational and technical details. In Sect. 3 we present the results of the qualitative analysis of obtained molecular line spectra, line parameters and abundance estimates. We discuss the obtained results in Sect. 4 and conclude in Sect. 5.

\section{Target and line selection, observations, and data reduction}

\subsection{Target selection}

A sample of 15 southern infrared dark clouds was selected by visual examination of the MSX $8.3 \mu \mathrm{m}$ images for the presence of high-contrast dark clouds. $1.2 \mathrm{~mm}$ continuum data were obtained for these objects with the SIMBA/SEST telescope. Together with millimeter data we used $8 \mu \mathrm{m}$ IRAC data from the Spitzer Galactic Legacy Infrared Mid-Plane Survey Extraordinaire (GLIMPSE, Benjamin et al. 2003) to investigate the physical properties of the extinction and emission material (see Paper I). Based on our millimeter and mid-IR data and taking into account single-dish beam sizes at millimeter wavelengths, we selected several points for observations in every cloud. The criterion for selection was the presence of either a $1.2 \mathrm{~mm}$ emission peak, a $8 \mu \mathrm{m}$ emission peak, or a $8 \mu \mathrm{m}$ extinction peak. These criteria let us cover IRDCs at the different evolutionary stages. Millimeter emission and mid-IR extinction indicate the presence of cold gas, which is typical for more quiescent regions. A typical source of $8 \mu \mathrm{m}$ emission usually contains a small infrared cluster and is therefore likely to be at a more advanced evolutionary stage than most dark regions in this IRAC band. The targets are listed in Table 1. Three-color Spitzer/GLIMPSE images of IRDCs together with the telescope and beam positions are presented in the on-line material Fig. A.1.

\subsection{Line selection}

In order to probe the dense and cold gas in IRDCs, we need appropriate tracers. The 3-mm band offers a large selection of molecular transitions. In particular, rotational transitions with low quantum numbers are accessible. Below we introduce the 3-mm lines we selected for our study.

$\mathbf{N}_{2} \mathbf{H}^{+}$: this species is known to be a selective tracer of quiescent gas (e.g., Caselli et al. 2002) and is particularly suitable for studying the structure and kinematics of cold star-forming cores. The hyperfine structure allows us to reliably measure the optical depth. $\mathrm{N}_{2} \mathrm{H}^{+}$is known to be a "late depleter", thus it is not strongly affected by freeze-out on grain surfaces (which prevents the use of the more common CO or CS transition). This makes it a robust tool for scrutinizing the highly structured interiors of massive star-forming regions where warm massive protostars could co-exist with younger cold and massive cloud cores. $\mathrm{N}_{2} \mathrm{H}^{+}$ has been - detected in both low- and high- mass pre-stellar cores and infrared dark clouds (e.g., Lee et al. 2001; Pirogov et al. 2003; Fuller et al. 2005; Ragan et al. 2006).

${ }^{13} \mathrm{CS}$ : this molecule is a very good tracer of dense gas (e.g., Bronfman et al. 1996) because of its high dipole moment. It was detected in low-mass pre-stellar cores (e.g., Tafalla et al. 2002) and high-mass star-forming regions (e.g., Jones et al. 2008). In contrast to $\mathrm{N}_{2} \mathrm{H}^{+}$, in cold dark clouds the CS emission vanishes toward the core center because of depletion, but it can be used to trace layers surrounding the central cores.

$\mathrm{CH}_{3} \mathbf{C N}$ : this molecule is considered to be a tracer of warm and dense regions (e.g., Kalenskii et al. 2000; Araya et al. 2005; Viti 2005). Chemical models involving only gas phase reactions and also models which take into account grain-surface chemistry show that $\mathrm{CH}_{3} \mathrm{CN}$ is only detectable in an environment with elevated temperatures (see Purcell et al. 2006 and references therein). It was detected in more evolved massive star-formation regions, such as Sgr B2 (Jones et al. 2008) or G305.2+0.2 (Walsh $\&$ Burton 2006), thus confirming the theoretical predictions. We include $\mathrm{CH}_{3} \mathrm{CN}$ in our "cold-gas-survey", because among infrared dark points we have quite a significant number of regions where star-formation processes may have already started.

$\mathbf{H C}_{\mathbf{3}} \mathbf{N}$ : this molecule belongs to an important group of interstellar molecules - the cyanopolyynes, $\mathrm{HC}_{2 n+1} \mathrm{~N}$. and is a valuable tracer of physical conditions in molecular clouds. Since its first detection in space (Turner 1971), $\mathrm{HC}_{3} \mathrm{~N}$ has been found in every type of molecular cloud from giant molecular clouds associated with $\mathrm{H}$ II regions to circumstellar envelopes. It was shown that $\mathrm{HC}_{3} \mathrm{~N}$ transitions have low optical depth and indicate the presence of denser gas than other high-density tracers (Bergin et al. 1996).

HNC: this molecule is a commonly used tracer of dense gas in molecular clouds. The abundance ratio $\mathrm{HCN} / \mathrm{HNC}$ strongly 
Table 1. List of observed IRDCs.

\begin{tabular}{|c|c|c|c|c|c|c|}
\hline Name & $\begin{array}{c}\text { RA } \\
(\mathrm{J} 2000.0)\end{array}$ & $\begin{array}{c}\text { Dec } \\
(\mathrm{J} 2000.0)\end{array}$ & $\begin{array}{c}\text { distance }^{a} \\
(\mathrm{kpc})\end{array}$ & $\begin{array}{l}T^{c} \\
(\mathrm{~K})\end{array}$ & $\begin{aligned} & N\left(\mathrm{H}_{2}\right)^{d} \\
\times & 10^{22} \mathrm{~cm}^{-2}\end{aligned}$ & category $^{e}$ \\
\hline IRDC308.13-1 & 133701.582 & -624434.01 & 4.8 & 13.5 & 1.0 & Q \\
\hline IRDC308.13-2 & 133700.418 & -624341.01 & 4.0 & 13.5 & 1.0 & $M$ \\
\hline IRDC308.13-3 & 133702.163 & -624339.01 & 4.0 & 13.5 & 1.0 & M \\
\hline IRDC309.13-1 & 134517.521 & -622202.84 & 3.9 & 16.3 & 0.6 & M \\
\hline IRDC309.13-2 & 134522.610 & -622327.48 & 3.9 & 14.7 & 0.5 & M \\
\hline IRDC309.13-3 & 134516.775 & -622537.25 & 3.9 & 35.4 & 0.4 & A \\
\hline IRDC309.37-1 & 134838.532 & -624617.55 & 3.4 & 31.4 & 0.9 & A \\
\hline IRDC309.37-2 & 134756.116 & -624833.46 & 3.4 & 15.7 & 0.8 & A \\
\hline IRDC309.37-3 & 134839.383 & -624722.39 & 3.4 & 15.7 & 0.7 & Q \\
\hline IRDC310.39-1 & 135601.359 & -621418.29 & 4.2 & 27.4 & 1.2 & A \\
\hline IRDC310.39-2 & 135600.759 & -621359.80 & 4.2 & 27.4 & 1.2 & A \\
\hline IRDC312.36-1 & 141127.752 & -612927.18 & 4.5 & 13.5 & 1.1 & A \\
\hline IRDC312.36-2 & 141156.773 & -612925.78 & 4.0 & 14.4 & 0.3 & Q \\
\hline IRDC313.72-1 & 142253.158 & -611441.00 & 3.3 & 19.9 & 0.4 & A \\
\hline IRDC313.72-2 & 142257.151 & -611410.84 & 3.3 & 19.9 & 0.4 & A \\
\hline IRDC313.72-3 & 142302.720 & -611339.64 & 3.3 & 19.9 & 0.3 & Q \\
\hline IRDC313.72-4 & 142304.533 & -611446.00 & 3.3 & 19.9 & 0.4 & Q \\
\hline IRDC316.72-1 & 144419.000 & -594429.00 & 2.7 & 26.1 & 1.2 & M \\
\hline IRDC316.76-1 & 144456.000 & -594808.00 & 2.7 & 22.6 & 4.1 & A \\
\hline IRDC316.72-2 & 144415.400 & -594320.00 & 2.7 & 24.3 & 1.3 & Q \\
\hline IRDC316.76-2 & 144500.500 & -594844.00 & 2.8 & 23.2 & 4.8 & A \\
\hline IRDC317.71-1 & 145106.905 & -591611.03 & 3.0 & 15.6 & 1.2 & Q \\
\hline IRDC317.71-2 & 145110.975 & -591701.73 & 3.0 & 16.6 & 3.5 & $\mathrm{~A}$ \\
\hline IRDC317.71-3 & 145119.667 & -591743.77 & 3.2 & 15.6 & 0.6 & Q \\
\hline IRDC320.27-1 & 150756.251 & -575432.11 & 2.1 & 15.3 & 0.8 & $\mathrm{Q}$ \\
\hline IRDC320.27-2 & 150731.616 & -575327.24 & 2.2 & 16.1 & 0.5 & Q \\
\hline IRDC320.27-3 & 150735.077 & -575413.98 & 2.1 & 16.1 & 0.4 & $\mathrm{Q}$ \\
\hline IRDC321.73-1 & 151826.387 & -572200.14 & 2.2 & 22.0 & 1.0 & M \\
\hline IRDC321.73-2 & 151801.693 & -572202.00 & 2.2 & 11.7 & 1.7 & M \\
\hline IRDC321.73-3 & 151801.065 & -572124.48 & 2.1 & 11.7 & 1.7 & A \\
\hline IRDC013.90-1 & 181733.378 & -170636.70 & 2.5 & 12.9 & 2.6 & M \\
\hline IRDC013.90-2 & 181719.350 & -170923.69 & 2.4 & 13.4 & 1.1 & Q \\
\hline IRDC316.45-1 & 144451.515 & -603055.00 & 3.1 & 15.4 & 0.7 & M \\
\hline IRDC316.45-2 & 144447.095 & -603130.89 & 3.1 & 14.2 & 0.5 & M \\
\hline IRDC318.15-1 & 145557.704 & -592904.12 & 3.0 & 17.6 & 0.5 & M \\
\hline IRDC318.15-2 & 145558.334 & -592830.52 & 2.9 & 17.6 & 0.4 & $\mathrm{Q}$ \\
\hline IRDC309.94-1 & 135054.970 & -614421.00 & $5.3^{b}$ & 48.8 & 5.2 & A \\
\hline
\end{tabular}

Notes. Columns are name, right ascension, declination, distance, kinetic temperature, $\mathrm{H}_{2}$ column density and type according to mid-IR classification. ${ }^{(a)}$ At first, to estimate the kinematic distances to our IRDCs, we used $\mathrm{HCO}^{+}$line velocities (see Vasyunina et al. 2009). But more detailed investigation showed that $\mathrm{HCO}^{+}$lines, as a rule, have complex line shapes and the $v_{\text {LSR }}$ positions can be shifted up to $2 \mathrm{~km} \mathrm{~s}^{-1}$ in comparison with its optically thin isotopologue $\mathrm{H}^{13} \mathrm{CO}^{+}$. We cannot use $\mathrm{H}^{13} \mathrm{CO}^{+}$for distance determination, since it is much weaker and we detected it not in all cases. Thus, we decided to use $\mathrm{N}_{2} \mathrm{H}^{+}$for the distance determination, which is distinguishable for all regions except IRDC309.37-2. Despite the significant difference in velocities (up to $2 \mathrm{~km} \mathrm{~s}^{-1}$ ) between $\mathrm{HCO}^{+}$and $\mathrm{N}_{2} \mathrm{H}^{+}$, the kinematic distances did not change drastically compared with the values in Paper I. ${ }^{(b)}$ From Saito et al. (2001). ${ }^{\left({ }^{c}\right)}$ Temperatures were derived based on $(1,1)$ and $(2,2)$ ammonia transitions observed with the the 64-m Parkes radio telescope (Linz et al., in prep.). ${ }^{(d)}$ To estimate $\mathrm{H}_{2}$ column densities in every point we used $1.2 \mathrm{~mm}$ data from SIMBA/SEST adopting the Mopra telescope beam size. (e) "A" indicates "active" cores, "M" - "middle", "Q" - "quiescent".

depends on the temperature and for the Orion molecular cloud decreases from 80 near the warm core to 5 on the colder edges (Goldsmith et al. 1986; Schilke et al. 1992). The recent theoretical work by Sarrasin et al. (2010) confirmed that this ratio should be around one for cold molecular clouds. This line is a triplet, but the spread of the hyperfine components is only $0.21 \mathrm{MHz}$ or $0.4 \mathrm{~km} \mathrm{~s}^{-1}$.

$\mathrm{HCO}^{+}$and $\mathrm{H}^{13} \mathrm{CO}^{+}: \mathrm{HCO}^{+}$is known to be a good tracer of the dense gas especially of embedded molecular outflows (e.g., Codella et al. 2001; Hofner et al. 2001). $\mathrm{HCO}^{+}$is an abundant molecule, with particularly enhanced abundances around regions of higher fractional ionization. It is also enhanced by outflows where shock-generated radiation fields are present (Rawlings et al. 2000, 2004).
The emission from the $\mathrm{H}^{13} \mathrm{CO}^{+}$isotopologue is mostly optically thin and traces similar gas densities as $\mathrm{HCO}^{+}$. A comparison between the generally optically thick $\mathrm{HCO}^{+}$line and the optically thin $\mathrm{H}^{13} \mathrm{CO}^{+}$line yields information on the bulk motion of the gas in the region.

HCN and $\mathbf{H}^{\mathbf{1 3}} \mathbf{C N}$ : this molecule has been suggested as a ubiquitous high-density gas tracer. Moreover, the HCN molecule is known to be a good tracer of infall motions in low-mass starforming regions. However, for the high-mass cores this can be different. Here HCN may become an unreliable infall tracer because of a higher level of turbulence (Redman et al. 2008) and outflow signatures (Zhang et al. 2007).

HNCO: Zinchenko et al. (2000) showed that HNCO integrated intensities correlate well with those of thermal SiO emission in massive clouds. This can indicate a spatial coexistence 
Table 2. Observed molecular species.

\begin{tabular}{|c|c|c|c|c|c|c|}
\hline Molecule & Transition & $\begin{array}{l}\text { Rest frequency } \\
(\mathrm{GHz})\end{array}$ & $\begin{array}{l}A \\
\left(\times 10^{-5} \mathrm{~s}^{-1}\right)\end{array}$ & $g_{\mathrm{u}}$ & $\begin{array}{l}E_{\mathrm{u}} \\
(\mathrm{K})\end{array}$ & Comments \\
\hline $\mathrm{CH}_{3} \mathrm{C}_{2} \mathrm{H}$ & $\begin{array}{l}5_{3}-4_{3} \\
5_{2}-4_{2} \\
5_{1}-4_{1} \\
5_{0}-4_{0}\end{array}$ & $\begin{array}{l}85.442600 \\
85.450765 \\
85.455665 \\
85.457299\end{array}$ & $\begin{array}{l}0.129778 \\
0.170373 \\
0.194760 \\
0.202908\end{array}$ & $\begin{array}{l}44 \\
22 \\
22 \\
22\end{array}$ & $\begin{array}{l}77.37 \\
41.22 \\
19.53 \\
12.30\end{array}$ & $\begin{array}{l}\text { Tracer of dense gas, } \\
\text { good thermometer }\end{array}$ \\
\hline $\mathrm{H}^{13} \mathrm{CN}$ & $\begin{array}{l}1_{1}-0_{1} \\
1_{2}-0_{1} \\
1_{0}-0_{1}\end{array}$ & $\begin{array}{l}86.338735 \\
86.340167 \\
86.342256\end{array}$ & 2.8 & 9 & 4.14 & $\begin{array}{l}\text { Tracer of dense gas, } \\
\text { infall motions }\end{array}$ \\
\hline $\mathrm{H}^{13} \mathrm{CO}^{+}$ & $1-0$ & 86.754330 & 2.8 & 3 & 4.16 & Tracer of dense gas \\
\hline $\mathrm{SiO}$ & $2-1$ & 86.847010 & 2.0 & 5 & 6.25 & Tracer of shocked gas \\
\hline $\mathrm{C}_{2} \mathrm{H}$ & $1-03 / 2-1 / 2 \quad F=2-1$ & 87.316925 & 0.152757 & 5 & 4.19 & $\begin{array}{l}\text { Tracer of early } \\
\text { stages of } \\
\text { star formation }\end{array}$ \\
\hline $\mathrm{HNCO}$ & $4_{0,4}-3_{0,3}$ & 87.925238 & 0.878011 & 9 & 10.55 & $\begin{array}{l}\text { Indicates denser gas than } \\
\text { other high-density tracers }\end{array}$ \\
\hline $\mathrm{HCN}$ & $\begin{array}{l}1_{1}-0_{1} \\
1_{2}-0_{1} \\
1_{0}-0_{1}\end{array}$ & $\begin{array}{l}88.6304157 \\
88.6318473 \\
88.6339360\end{array}$ & 2.4 & 9 & 4.25 & $\begin{array}{l}\text { Tracer of dense gas, } \\
\text { infall motions }\end{array}$ \\
\hline $\mathrm{HCO}^{+}$ & $1-0$ & 89.188526 & 3.0 & 3 & 4.28 & $\begin{array}{l}\text { Tracer of dense gas, } \\
\text { outflows }\end{array}$ \\
\hline $\mathrm{HNC}$ & $\begin{array}{l}1_{0}-0_{1} \\
1_{2}-0_{1} \\
1_{1}-0_{1}\end{array}$ & $\begin{array}{l}90.663450 \\
90.663574 \\
90.663656\end{array}$ & 2.7 & 3 & 4.35 & Tracer of dense gas \\
\hline $\mathrm{HC}_{3} \mathrm{~N}$ & $10-9$ & 90.978989 & 5.81300 & 21 & 24.02 & $\begin{array}{l}\text { Indicates denser gas than } \\
\text { other high-density tracers }\end{array}$ \\
\hline $\mathrm{CH}_{3} \mathrm{CN}$ & $\begin{array}{l}5_{4}-4_{4} \\
5_{3}-4_{3} \\
5_{2}-4_{2} \\
5_{1}-4_{1} \\
5_{0}-4_{0}\end{array}$ & $\begin{array}{l}91.959206 \\
91.971465 \\
91.980089 \\
91.985316 \\
91.987089\end{array}$ & $\begin{array}{l}2.27824 \\
4.05228 \\
5.31863 \\
6.07995 \\
6.33432\end{array}$ & $\begin{array}{l}22 \\
44 \\
22 \\
22 \\
22\end{array}$ & $\begin{array}{l}127.60 \\
77.58 \\
41.84 \\
20.39 \\
13.25\end{array}$ & $\begin{array}{l}\text { Tracer of warm and } \\
\text { dense regions }\end{array}$ \\
\hline${ }^{13} \mathrm{CS}$ & $2-1$ & 92.494303 & 1.41254 & 10 & 6.66 & Tracer of dense gas \\
\hline $\mathrm{N}_{2} \mathrm{H}^{+}$ & $\begin{array}{l}1_{11}-0_{01} \\
1_{11}-0_{22} \\
1_{11}-0_{10} \\
1_{21}-0_{21} \\
1_{21}-0_{32} \\
1_{21}-0_{11} \\
1_{01}-0_{12}\end{array}$ & $\begin{array}{l}93.171621 \\
93.171917 \\
93.172053 \\
93.173480 \\
93.173777 \\
93.173967 \\
93.176265\end{array}$ & 3.8534 & 27 & 4.47 & $\begin{array}{l}\text { Tracer of quiescent gas, } \\
\text { "late depleter" }\end{array}$ \\
\hline
\end{tabular}

Notes. Columns are species, transition, rest frequency, Einstein A coefficients, degeneracy, energy of the upper level, comments.

of the two species and may hint at a common production mechanism, presumably based on shock chemistry.

$\mathbf{C}_{2} \mathbf{H}$ : chemical models predict that $\mathrm{C}_{2} \mathrm{H}$ is only well centered on the sources when they are very young. At later stages it is destroyed in the central cores, but is replenished in the outer shells (Beuther et al. 2008).

SiO: it can trace shocked gas potentially associated with energetic young outflows. Hence, this line can reveal starformation activity even for cores where no Spitzer/MIPS sources are apparent (e.g., Lo et al. 2007). For a few of our IRDCs we have indications from Spitzer/GLIMPSE imaging that shocked gas exists, often, but not exclusively, at the edges of the IRDCs. Tracers are the "green fuzzies" found in the $4.5 \mu \mathrm{m}$ GLIMPSE channel (Cyganowski et al. 2008; Chambers et al. 2009) and generally attributed to pure rotational IR lines of $\mathrm{H}_{2}$ (De Buizer \& Vacca 2010).

$\mathrm{CH}_{3} \mathbf{C}_{2} \mathbf{H}$ : a good tracer of dense gas appropriate for early stages of star-forming regions (Bergin et al. 1994). This molecule can be used as a good thermometer in a dense environment.

The covered molecular lines and transitions are summarized in Table 2.

\subsection{Mopra observations and data reduction}

The observations were made with the 22-m Mopra radio telescope, operated by the Australia Telescope National Facility (ATNF) in position switching mode. In total we spent 7 min on source and $7 \mathrm{~min}$ on the OFF position. Our targets are dense molecular condensations within larger molecular clouds with often widespread molecular emission. Therefore, we refrained from using one standard OFF position throw. Instead, OFF positions were chosen individually for every target region and were approximately $8-10^{\prime}$ away from the source.

The Mopra spectrometer (MOPS) offers zoom-mode configurations with the possibility to observe up to 16 sub-bands 
of $138 \mathrm{MHz}$ each within a total frequency range of $8.3 \mathrm{GHz}$. This set-up delivers a velocity resolution of $\sim 0.11 \mathrm{~km} \mathrm{~s}^{-1}$.

The observations were carried out on 9-11 May 2008 with the $3 \mathrm{~mm}$ band receiver. We put the central frequency for the $8.3 \mathrm{GHz}$ block to $89270 \mathrm{MHz}$ and thus covered the range from 85 to $93 \mathrm{GHz}$. In this range we distributed 13 zoom windows, which covered the lines listed in Table 2.

System temperature measurements were performed every $30 \mathrm{~min}$ and a pointing scan every hour. Typical system temperatures (measured with the common chopper-wheel technique) during the observations were $170-210 \mathrm{~K}$. At Mopra observatory, $\mathrm{SiO}$ masers are used to correct the telescope pointing, giving a pointing accuracy better than $10^{\prime \prime}$. The main beam of the telescope varies between $36^{\prime \prime}$ at $86 \mathrm{GHz}$ and $33^{\prime \prime}$ at $115 \mathrm{GHz}$ and the main beam efficiency varies between 0.49 at $86 \mathrm{GHz}$ and 0.44 at $100 \mathrm{GHz}$ (Ladd et al. 2005)

Mopra data are originally stored in RPFITS format. Using the ATNF Spectral line Analysis package (ASAP), we transformed these raw data into ascii files, which were then fed into GILDAS for further analysis. The typical rms level in the obtained spectra is about $0.12-0.16 \mathrm{~K}$. We give $1 \sigma$ errors in Tables C.1-C.8.

\section{Results}

\subsection{Qualitative assessment}

Figure 1 presents the average spectrum of an infrared dark cloud. To produce it we shifted the spectra of all our IRDCs to the same reference velocity and averaged spectra for each species with equal weight. The "average spectra" technique enables us to reduce the noise level and recognize weak lines, which are not detectable in the single spectra. Spectra for every single point are available in the on-line material (see Fig. B.1). For all IRDCs we have quite strong and clear detections of the $\mathrm{HNC}, \mathrm{HCO}^{+}$ and $\mathrm{HCN}$ molecules. In all cases we see a non-Gaussian shape of $\mathrm{HCO}^{+}$with "shoulders" or double-peaked profiles. We will discuss the asymmetries of $\mathrm{HCO}^{+}$in comparison with the optically thin $\mathrm{H}^{13} \mathrm{CO}^{+}$line in Sect. 3.3 in more detail.

$\mathrm{N}_{2} \mathrm{H}^{+}$is detected everywhere, except for IRDC309.37-2. As expected, we cannot distinguish all seven hyperfine components because of the large line width. While one hyperfine component $\left(1_{01}-0_{12}\right)$ constitutes a distinct line peak, the other six transitions merge into two satellites.

$\mathrm{HC}_{3} \mathrm{~N}, \mathrm{HNCO}$ and $\mathrm{C}_{2} \mathrm{H}$ show rather weak emission and were detected in 18, 13, and 24 positions, respectively.

According to the previous studies (e.g. Ragan et al. 2006; Jackson et al. 2008), CS is a common molecule in IRDCs. Still, we detected only very weak ${ }^{13} \mathrm{CS}(2-1)$ emission in three clouds. The low detection rate of ${ }^{13} \mathrm{CS}(2-1)$ in our case can be explained by 50 times less abundant ${ }^{13} \mathrm{CS}$ in comparison with CS. Also depletion of CS (and ${ }^{13} \mathrm{CS}$ ) can play a significant role here (Beuther \& Henning 2009). Another molecule, $\mathrm{CH}_{3} \mathrm{C}_{2} \mathrm{H}$, was detected only in IRDC316.76-1 and IRDC317.71-2 and shows very weak emission in IRDC316.72-1, IRDC316.72-2 and IRDC316.76-2.

Even in the most evolved regions IRDC316.76-1 and IRDC316.76-2, where there is strong mid-IR and millimeter emission, we detected no $\mathrm{CH}_{3} \mathrm{CN}$ as a typical hot core tracer. Because $\mathrm{CH}_{3} \mathrm{CN}$ emission arises from a warm and compact region, one explanation of this non-detection can be the low spatial resolution of the telescope and the relatively low signal-to-noise ratio in our spectra. Using the "average spectra" technique, we were able to reduce the noise level and to find very weak $\mathrm{CH}_{3} \mathrm{CN}$ emission (see Fig. 1). This detection indicates that $\mathrm{CH}_{3} \mathrm{CN}$ is

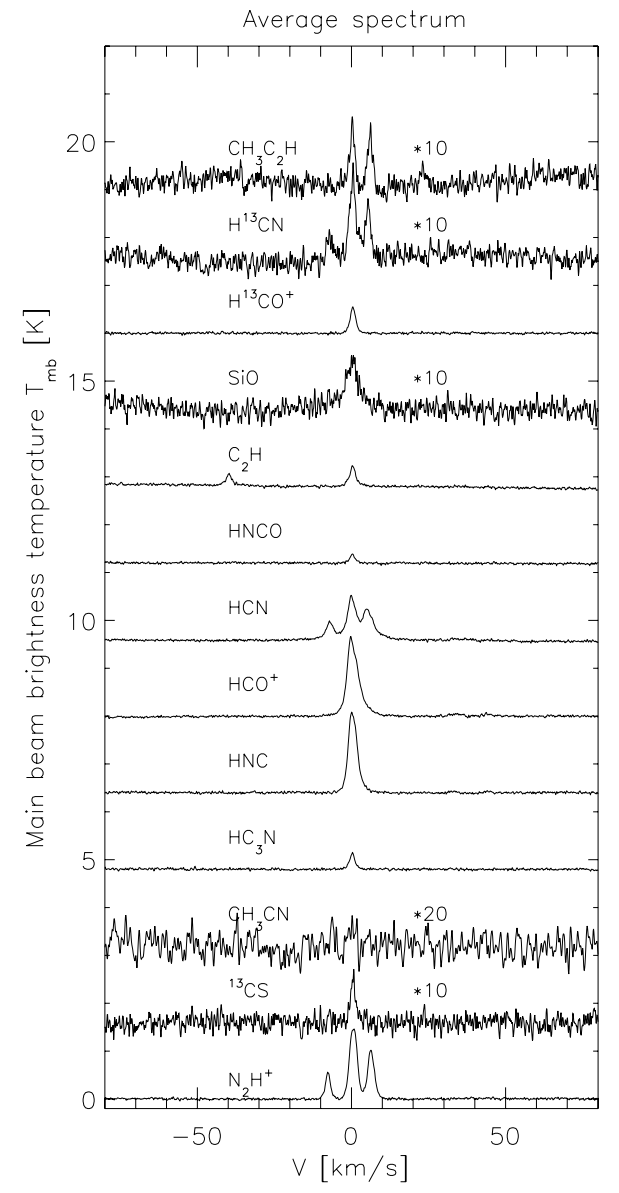

Fig. 1. Average spectrum. Obtained by averaging all spectra for every species with equal weight after shifting all of them to the same reference velocity. Several weak detections are amplified by a factor of 10 , and $\mathrm{CH}_{3} \mathrm{CN}$ is amplified by a factor of 20 for plotting.

present at least in some parts of our clouds, but there is an abundance deficit for this molecule in IRDCs. This result agrees with the previous work by Beuther \& Sridharan (2007).

According to Cyganowski et al. (2008) and Chambers et al. (2009) it is more likely to see $\mathrm{SiO}$ as an outflow tracer in the clouds where there is emission both at 4.5 and $24 \mu \mathrm{m}$. However, we detected $\mathrm{SiO}$ lines with extended line wings only in three regions with mid-IR emission: IRDC313.72-1, IRDC313.72-2 and IRDC316.76-1. Other potentially interesting regions with emission in mid-IR either show very weak $\mathrm{SiO}$ emission (IRDC317.71-2, IRDC309.94-1), or no SiO emission at all (IRDC309.37-1, IRDC310.39-2). The strongest SiO line was detected in the IRDC321.73-1. Within the Mopra beam this region contains a very weak source at $24 \mu \mathrm{m}$ and emission at GLIMPSE $4.5 \mu \mathrm{m}$ so weak that it was not identified as extended green objects (EGOs) in Cyganowski et al. (2008). We classify this object as "middle" (see below). Detection of $\mathrm{SiO}$ emission in this source indicates that absence or extreme weakness of midIR emission does not mean that star-formation processes are not taking place in a cloud (cf. Lo et al. 2007) and shows the necessity of spectral line observations to identify outflows and starformation activity in molecular clouds.

Based on mid-infrared Spitzer data, Chambers et al. (2009) subdivided infrared dark clouds cores into "active" and "quiescent". Cores were classified as "active" if they showed emission both at 8 and $24 \mu \mathrm{m}$, and as "quiescent" if they contained neither 

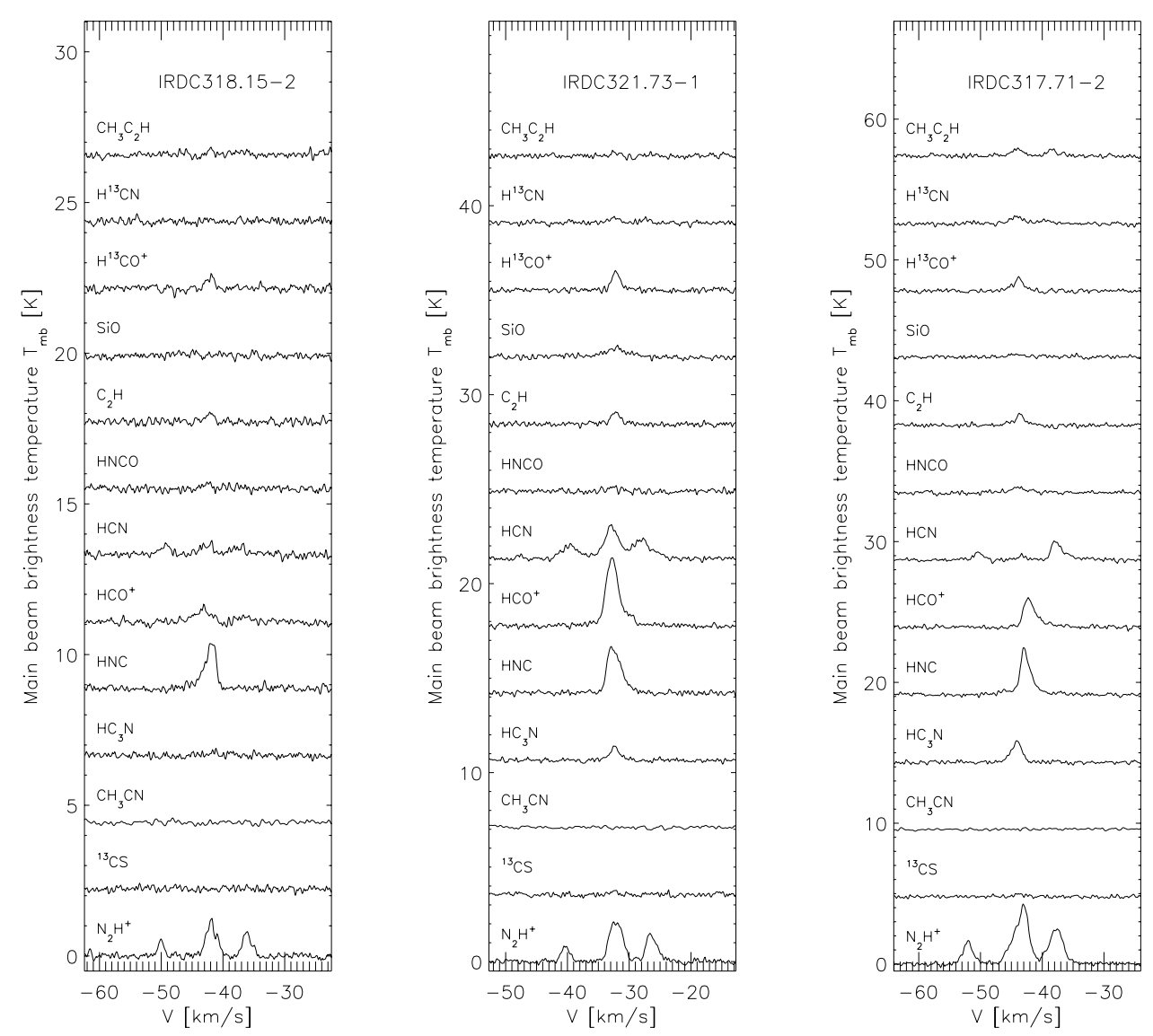

Fig. 2. Line spectra for different mid-IR categories of IRDCs. (left) "Quiescent" cloud, (middle) "middle" cloud, (right) "active" cloud.

IR emission signatures. We added a "middle" stage to this classification whenever detect emission at 24 micron only and not at the shorter wavelength (see Table 1). In the Fig. 2 we present the spectra for typical clouds from every category. Chambers et al. (2009) have shown that "active" cores have smaller sizes, higher densities, and more pronounced water and methanol maser activity than the "quiescent" cores. However, from the qualitative analysis of the Mopra spectra, we do not see clear molecular signatures of any of these three categories of sources.

\subsection{Line parameters}

Using the GILDAS/CLASS software, we fitted the whole $\mathrm{N}_{2} \mathrm{H}^{+}$ structure, which resulted in local standard of rest (LSR) velocities, full linewidths at half maximum, and optical depths (Tables C.1). For the lines where there is no hyperfine splitting $\left({ }^{13} \mathrm{CS}, \mathrm{HC}_{3} \mathrm{~N}, \mathrm{HCO}^{+}, \mathrm{HNCO}, \mathrm{SiO}, \mathrm{H}^{13} \mathrm{CO}^{+}\right)$, or the hyperfine structure cannot be resolved (HNC), we estimate integrated and peak line intensities, LSR velocities and full linewidths at half maximum from Gaussian fits (Tables C.2-C.8). For all lines we estimated integrated areas, measured by summing the channels between suitable velocity limits under the line (Table C.9). To compare our results with the line parameters of other IRDCs, low-mass starless cores and more evolved high-mass clouds we chose $\mathrm{N}_{2} \mathrm{H}^{+}$. This line has a relatively simple line shape without extended shoulders and self-absorption features, and is detected in $97 \%$ of the clouds. Moreover, $\mathrm{N}_{2} \mathrm{H}^{+}$is widely detected in low- and high-mass pre-stellar and protostellar cores.

We find that the line widths for our IRDCs vary in the range from 0.6 to $2.8 \mathrm{~km} \mathrm{~s}^{-1}$ and agree with the results obtained for

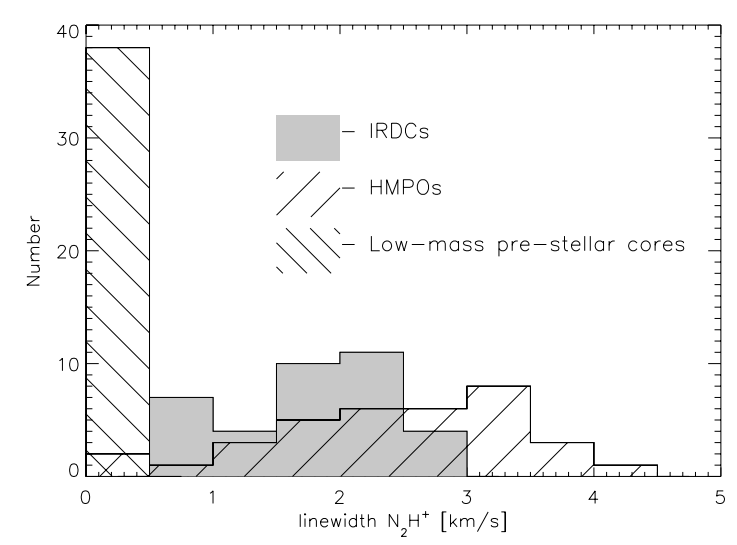

Fig. 3. Distribution of $\mathrm{N}_{2} \mathrm{H}^{+}$line width for our IRDCs sample, more evolved regions from Pirogov et al. (2003) and low-mass pre-stellar cores from Lee et al. (2001).

other IRDCs by Ragan et al. (2006), where the line width was $0.5-4.0 \mathrm{~km} \mathrm{~s}^{-1}$ with the mean value $2.2 \mathrm{~km} \mathrm{~s}^{-1}$. In low-mass pre-stellar cores (Lee et al. 2001) strong $\mathrm{N}_{2} \mathrm{H}^{+}$emission was detected in $75 \%$ of the objects. Lines in low-mass regions are narrow with line widths in the range of $\sim 0.2-0.4 \mathrm{~km} \mathrm{~s}^{-1}$ and a mean value of $0.3 \mathrm{~km} \mathrm{~s}^{-1}$, which corresponds to the thermal linewidth. High-mass protostellar objects show $\mathrm{N}_{2} \mathrm{H}^{+}$emission in all selected targets (Pirogov et al. 2003; Fuller et al. 2005). Compared with low-mass cores, these targets have broader lines $0.5-3.5 \mathrm{~km} \mathrm{~s}^{-1}$. Figure 3 presents the distribution of the $\mathrm{N}_{2} \mathrm{H}^{+}$line width for all three types of objects. 


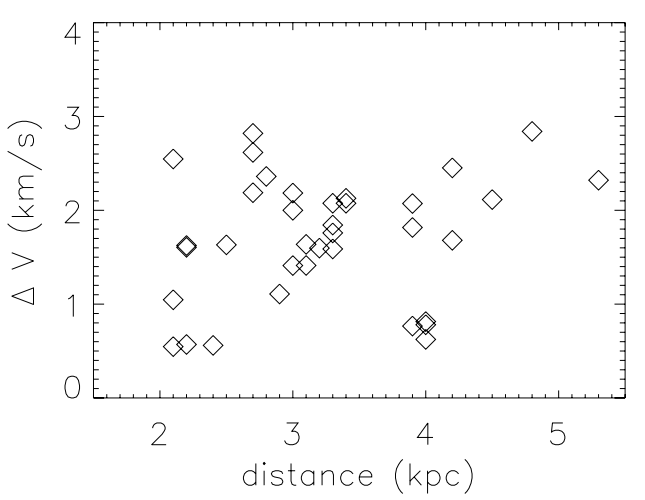

Fig. 4. Comparison between $\mathrm{N}_{2} \mathrm{H}^{+}$line width and kinematic distance to the objects for our IRDCs sample.

Broader IRDC lines can indicate more turbulent processes within clouds. However, a $36^{\prime \prime}$ beam corresponds to $0.17 \mathrm{pc}$ at $1 \mathrm{kpc}$ distance and $0.9 \mathrm{pc}$ at $5 \mathrm{kpc}$. Thus, increasingly higher volumes are covered by the same beam for regions farther away and may therefore offer a simple explanation for larger line widths. To check if the line widths become larger for more distant clouds, we plot in Fig. 4 the line width against the kinematic distance. For this comparison we use our $\mathrm{N}_{2} \mathrm{H}^{+}$data from the Table C.1. For our objects the correlation coefficient between the two parameters is only 0.2 . Thus, large line widths of high-mass clouds cannot be explained exclusively by the large distances. In the same vein, clumping, which can easily be hidden within a $36^{\prime \prime}$ beam, can only partly explain these elevated line widths (Pillai et al. 2006). The linewidth - size relationship also cannot explain high line width values in IRDCs. Heyer et al. (2001) report that a clear line width - size relation is only observed in larger clouds complexes of $>10 \mathrm{pc}$ in size. The IRDC clumps targeted by us are much smaller with typical sizes of around 1 pc. Furthermore, previous studies have already indicated that the line widths in high-mass star-forming clumps are much higher than would be predicted by the usual relations between line width and size determined from regions of lower mass (e.g. Shirley et al. 2003).

Comparing line parameters for "quiescent", "middle" and "active" regions, we can see that there is a trend to have larger line widths and higher integrated intensities in more evolved objects. The mean line widths are $1.4,1.7$ and $2.2 \mathrm{~km} \mathrm{~s}^{-1}$ and the integrated intensities are 4.7, 7.1 and $20.6 \mathrm{~K} \mathrm{~km} \mathrm{~s}^{-1}$ for "quiescent", "middle" and "active" sources respectively. These results agree well with Chambers et al. (2009), who concluded that "active" sources are more evolved and present further evolutionary stages compared with "quiescent" sources.

We also utilized $\mathrm{N}_{2} \mathrm{H}^{+}$to estimate kinematic distances to our objects (see Table 1). For IRDC309.37-2 the $\mathrm{N}_{2} \mathrm{H}^{+}$line was not detected and we used $\mathrm{HCO}^{+}$to estimate the distance. Because we observed several points within one cloud, the distance determination helps us to confirm that clumps with associated midIR emission in IRDC309.37, IRDC310.39 and IRDC371.71 are connected with the other "dark" parts of the respective clouds and are not only a projection effect.

\section{3. $\mathrm{HCO}^{+}$and $\mathrm{H}^{13} \mathrm{CO}^{+}$line profiles}

As already mentioned, the $\mathrm{HCO}^{+}$lines show a non-Gaussian profile in almost all clouds. In some cases even its isotopoloque

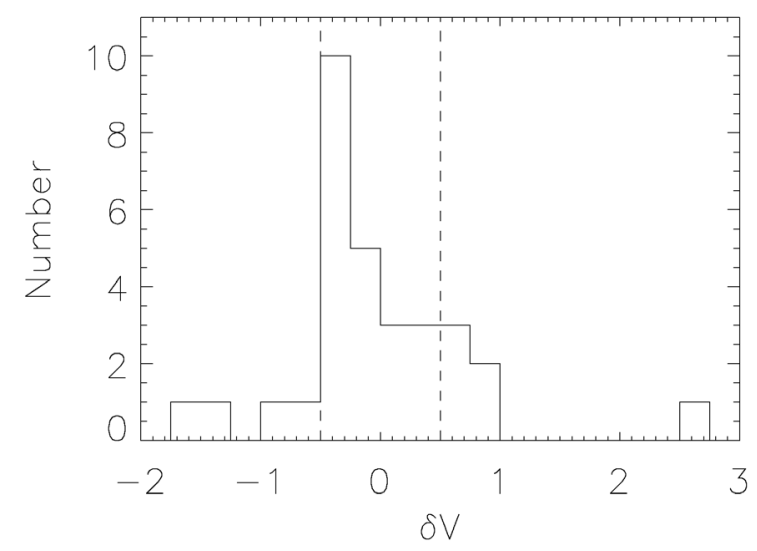

Fig. 5. Number distribution of $\delta V=\left(V_{\mathrm{HCO}^{+}}-V_{\mathrm{H}^{13} \mathrm{CO}^{+}}\right) / \Delta V_{\mathrm{H}^{13} \mathrm{CO}^{+}}$for our sample (see Sect. 3.3). Dashed lines indicate the $\pm 5 \sigma_{\delta \mathrm{V}}$ level.

$\mathrm{H}^{13} \mathrm{CO}^{+}$has a complicated line shape. Moreover, we see a significant shift, up to $2 \mathrm{~km} \mathrm{~s}^{-1}$, between the peak positions of the optically thick $\mathrm{HCO}^{+}$line and optically thin $\mathrm{H}^{13} \mathrm{CO}^{+}$. A quantitative estimate of this asymmetry could provide us with information about dynamical processes in the clouds.

The most common way to extract line asymmetries is based on the comparison of optically thin and optically thick line positions: $\delta V=\left(V_{\text {thick }}-V_{\text {thin }}\right) / \Delta V_{\text {thin }}$ (Mardones et al. 1997). This estimate was widely used both for low- and high-mass star-formation regions. (Fuller et al. 2005; Pirogov et al. 2003; Purcell et al. 2006; Lee et al. 2001). With this criterion, we can subdivide our IRDCs in to "blue shifted" clouds with $\delta V<0$ and "red shifted" clouds with $\delta V>0$. The blue excess could be caused by infall motions and the red excess by expanding motions or outflow. Among the sources where both $\mathrm{HCO}^{+}$and $\mathrm{H}^{13} \mathrm{CO}^{+}$were detected, we identified 19 "blue shifted" and 12 "red shifted" clouds (Fig. 5, Table C.8). In order to exclude the sources where differences between the $V_{\text {lsr }}$ values for different species are dominated by measurement errors, we have to determine the threshold value. For the low-mass starless cores Mardones et al. (1997) determined this threshold as $5 \sigma$, where $\sigma$ is the typical $\delta \mathrm{V}$ error. In our case $\mathrm{H}^{13} \mathrm{CO}^{+}$, which we use as optically thin component, is quite weak for some sources, which leads to the $5 \sigma$ value equal 0.5 . Most of our clouds have $|\delta V|<0.5$, only four objects show a sufficient blue shift, and six a red shift. From these 10 candidates we also have to exclude sources with very weak and/or noisy spectra. After this selection we determine one infall candidate: IRDC313.72-4, and three sources show evidence of expanding motions: IRDC317.71-1, IRDC317.71-2 and IRDC317.71-3. However, this method allows us only to identify infall or outflow candidates, but does not provide any quantitative values (e.g. infall speed).

Another method to estimate line asymmetries quantitatively was described in Myers et al. (1996). They used an analytic twolayer radiative transfer model to obtain theoretical spectra, compare them with the wide range of observed double-peak profiles and extract a formula for infall speed (see Eq. (9) from Myers et al. 1996). This method allows us to estimate the infall velocity in a cloud by measuring blue and red peak parameters of the optically thick line. Among all our targets we selected three clouds with clear red shoulders in $\mathrm{HCO}^{+}$and non-complicated shape of $\mathrm{H}^{13} \mathrm{CO}^{+}$(see Fig. 6). The estimated infall velocities are around $2 \mathrm{~km} \mathrm{~s}^{-1}$. This value is much higher than the sound speed 

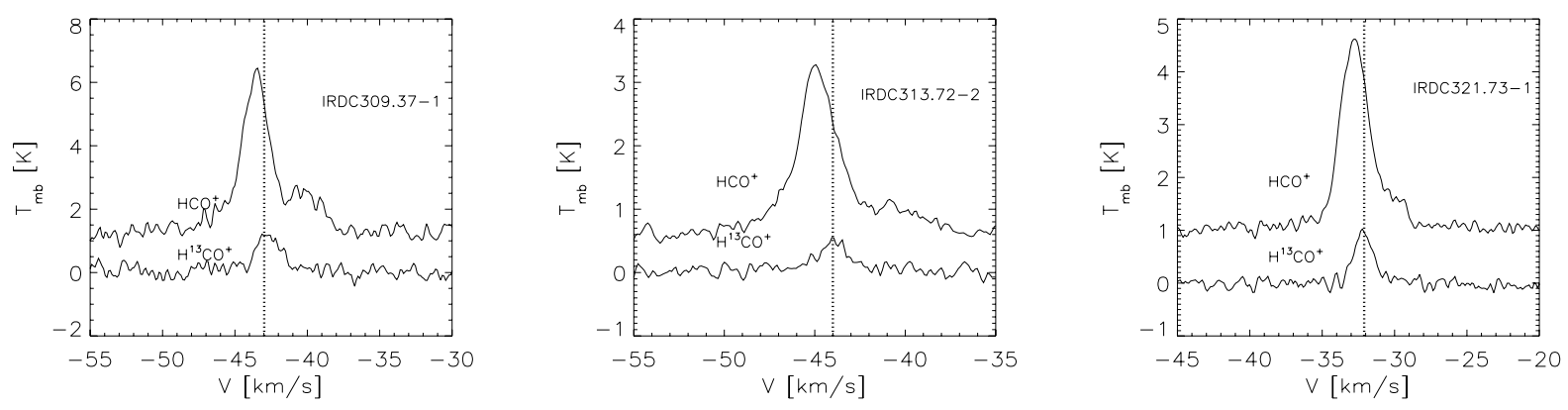

Fig. 6. Samples of the $\mathrm{HCO}^{+}$and $\mathrm{H}^{13} \mathrm{CO}^{+}$line profiles. The vertical lines indicate the $V_{\mathrm{lsr}}$ positions measured from $\mathrm{H}^{13} \mathrm{CO}^{+}$line profiles.

in such an environment $-0.3 \mathrm{~km} \mathrm{~s}^{-1}$, or a typical infall speed for low-mass pre-stellar cores $0.05-0.09 \mathrm{~km} \mathrm{~s}^{-1}$ (Lee et al. 2001).

\subsection{Derived quantities: column densities and abundances}

To estimate column densities we assume LTE conditions and optically thin emission and use the equation

$$
\begin{aligned}
N_{\text {tot }}= & \frac{8 \pi}{\lambda^{3} A} \frac{g_{1}}{g_{\mathrm{u}}} \frac{1}{J_{v}\left(T_{\mathrm{ex}}\right)-J_{v}\left(T_{\mathrm{bg}}\right)} \frac{1}{1-\exp \left(-h v / k T_{\mathrm{ex}}\right)} \\
& \times \frac{Q_{\mathrm{rot}}}{g_{1} \exp \left(-E_{\mathrm{l}} / k T_{\mathrm{ex}}\right)} \int T_{\mathrm{mb}} \mathrm{d} v,
\end{aligned}
$$

where $\lambda$ is the rest wavelength of the transition, $A$ is the Einstein coefficient, $g_{\mathrm{u}}$ is the upper state degeneracy, $J_{v}\left(T_{\mathrm{ex}}\right)$ and $J_{v}\left(T_{\mathrm{bg}}\right)$ are the equivalent Rayleigh-Jeans excitation and background temperatures, $Q_{\text {rot }}$ is the partition function, and $E_{1}$ is the energy of the lower level (Lee et al. 2009). In our calculations for $g_{\mathrm{u}}, A$ and $E_{1}$ we used values from The Cologne Database for Molecular Spectroscopy (CDMS) (Müller et al. 2001, 2005) (Table 2). For the excitation temperatures $T_{\text {ex }}$ in all cases, except $\mathrm{HCO}^{+}$and $\mathrm{H}^{13} \mathrm{CO}^{+}$(see below), we assume ammonia kinetic temperatures (Linz et al., in prep.) (Table 1). We calculate the partition function $Q_{\text {rot }}$ for every source by interpolating data from the CDMS for the particular source temperature $T_{\mathrm{ex}}$.

Integrated intensities $\int T_{\mathrm{mb}} \mathrm{d} v$ for every species were measured by summing the channels between suitable velocity limits under the corresponding molecular line (Table C.9).

The optically thin assumption is suitable for most of our lines: ${ }^{13} \mathrm{CS}, \mathrm{HC}_{3} \mathrm{~N}, \mathrm{HNCO}, \mathrm{C}_{2} \mathrm{H}, \mathrm{SiO}, \mathrm{H}^{13} \mathrm{CO}^{+}, \mathrm{H}^{13} \mathrm{CN}$, $\mathrm{CH}_{3} \mathrm{C}_{2} \mathrm{H}$. For optically thick emission (e.g. $\mathrm{HCN}, \mathrm{HCO}^{+}, \mathrm{HNC}$ ) Eq. (1) gives only a lower limit. However, for $\mathrm{N}_{2} \mathrm{H}^{+}$and $\mathrm{HCO}^{+}$ we can make a better column density estimation. In the case of $\mathrm{N}_{2} \mathrm{H}^{+}$we use the advantage of the hyperfine structure and estimate optical depths from the hyperfine components fitting. Then column densities for $\mathrm{N}_{2} \mathrm{H}^{+}$can be calculated by multiplying Eq. (1) by a factor of $\tau /\left(1-\mathrm{e}^{-\tau}\right)$. For $\mathrm{HCO}^{+}$the presence of an optically thin isotopoloque allows us to estimate more realistic values for $\mathrm{HCO}^{+}$. Using an algorithm described in Purcell et al. (2006) and assuming that $\mathrm{HCO}^{+}$is optically thick, $\mathrm{H}^{13} \mathrm{CO}^{+}$is optically thin and their relative abundance ratio $\mathrm{X}=\left[\mathrm{HCO}^{+}\right] /\left[\mathrm{H}^{13} \mathrm{CO}^{+}\right]$equals 50 , we calculated $\mathrm{HCO}^{+}$column densities for the sources, where both $\mathrm{HCO}^{+}$and $\mathrm{H}^{13} \mathrm{CO}^{+}$ lines were detected. We do not apply the same algorithm for the pair $\mathrm{HCN}-\mathrm{H}^{13} \mathrm{CN}$, because $\mathrm{H}^{13} \mathrm{CN}$ is detected only in four objects. In spite of non-detection of $\mathrm{CH}_{3} \mathrm{CN}$ in any single spectra, the "average spectra" technique lets us recognize the very weak $\mathrm{CH}_{3} \mathrm{CN}\left(5_{0}-4_{0}\right)$ and $\mathrm{CH}_{3} \mathrm{CN}\left(5_{1}-4_{1}\right)$ lines (see Fig. 1). We use this averaged spectrum to estimate the integrated intensity $\int T_{\mathrm{mb}} \mathrm{d} v$ and derive the upper limit column density for this species.

To transfer column densities to abundances we use the $N$ (species) $/ N\left(\mathrm{H}_{2}\right)$ ratio, where $N\left(\mathrm{H}_{2}\right)$ values were estimated from the $1.2 \mathrm{~mm}$ SIMBA/SEST data (Paper I) adopting the Mopra telescope beam size (see Table 1). For $\mathrm{CH}_{3} \mathrm{CN}$ we use the average value for the molecular hydrogen column density of $1.2 \times 10^{22} \mathrm{~cm}^{-2}$ and derived upper limit for $\mathrm{CH}_{3} \mathrm{CN}$ abundances of 4.0. $\times 10^{-11}$. Abundances for other species are presented in Table 3.

For our column density and abundance estimates, systematic errors play the dominant role. One aspect is the assumption that all lines are optically thin. For moderately optically thick lines, the true column densities may be higher by a factor of 2-3 for our objects. We also utilize kinetic temperatures derived from ammonia observations as excitation temperatures. This can give another factor of 2-5 to the estimated values. Systematic errors are also present in the molecular hydrogen column densities, derived from the millimeter continuum measurements, where we made assumptions about the dust model and equal gas and dust temperatures. All these assumptions give rise to a systematic error of an order of magnitude in molecular abundances which we obtained.

\subsection{Are the objects really cold?}

To estimate kinetic temperatures in our clouds we observed the ammonia $(1,1)$ and $(2,2)$ inversion transitions with the Parkes radio telescope. These transitions are known as a good thermometer for cold, dense gas. We derived kinetic temperatures for most IRDCs between 10 and $30 \mathrm{~K}$ (Linz et al., in prep.). Ammonia observations show cold gas in IRDCs. However, the Parkes telescope beam is $72^{\prime \prime}$ or approximately $1 \mathrm{pc}$ at the typical IRDCs distances. With the Mopra telescope (telescope beam 36") we can trace a lower volume and use other molecular lines to identify warmer regions.

One way is to consider the $\mathrm{HCN} / \mathrm{HNC}$ abundance ratio. This ratio strongly depends on the temperature and for the Orion molecular cloud decreases from 80 near the warm core to 5 on the colder edges (Goldsmith et al. 1986; Schilke et al. 1992; Sarrasin et al. 2010). For our IRDC sample the estimated $\mathrm{HCN} / \mathrm{HNC}$ abundance ratio values are not higher than 3 . This confirms that we deal with quite cold clouds.

Besides ammonia, symmetric top molecules like $\mathrm{CH}_{3} \mathrm{C}_{2} \mathrm{H}$ can be used as probes of kinetic temperature in molecular clouds (Bergin et al. 1994). We detect $\mathrm{CH}_{3} \mathrm{C}_{2} \mathrm{H}$ in 5 targets. In two objects the $5_{2}-4_{2}, 5_{1}-4_{1}$ and $5_{0}-4_{0}$ components are clearly visible. This allows us to estimate rotational temperatures in these regions with the algorithm given in Bergin et al. (1994). 
Derived temperatures equal $17 \mathrm{~K}$ for IRDC317.71-2 and $38 \mathrm{~K}$ for IRDC316.76-1. The temperature for IRDC317.71-2 is similar to the value we have from ammonia observations, whereas the temperature for IRDC $316.76-1$ is $15 \mathrm{~K}$ higher. This indicates that in some clouds a warmer component can be present in addition to cold gas. If there is also a warm gas component and the real gas temperature in some targets is higher, we underestimated molecular abundances. The abundances for IRDC316.761 with $T=38 \mathrm{~K}$ become a factor of 1.2-1.7 higher. However, taking into account all our assumptions and the still large telescope beam, this difference is still within the error limit.

\subsection{Special objects}

In this subsection we present infrared dark clouds where the line spectra show some unique features.

IRDC317.71 One of the remarkable features in this object is the ratio of the $\mathrm{HCN}$ hyperfine components, which are different from other IRDCs (see Fig. 2). In contrast to $\mathrm{N}_{2} \mathrm{H}^{+}$, the $\mathrm{HCN}$ hyperfine component intensity ratio is not a constant value. Afonso et al. (1998) show for low-mass dark clouds that HCN hyperfine components can have different intensity ratios in different sources. For most of our IRDCs we detected that the ratios $F=2-1$ to $F=0-1$ and $F=2-1$ to $F=1-1$ components are different, but higher than 1. For IRDC317.71-1 these ratios are 0.8 and 0.4 and for IRDC317.71-2 are 0.5 and 0.2 respectively.

Another interesting feature is the large shift between $\mathrm{H}^{13} \mathrm{CO}^{+}$and $\mathrm{HCO}^{+}$emission peaks. $\mathrm{H}^{13} \mathrm{CO}^{+}$emission peak is shifted to the blue side compared with $\mathrm{HCO}^{+}$and corresponds to the extinction in the optically thick component. Also IRDC317.71-2 is one of the five locations where $\mathrm{CH}_{3} \mathrm{C}_{2} \mathrm{H}$ was detected. As we can see from the previous section, the $\mathrm{CH}_{3} \mathrm{C}_{2} \mathrm{H}$ excitation analysis shows the presence of a warmer gas component, thus indicating a more evolved star-forming region.

Most probably the HCN spectrum can be explained by foreground absorption in low-excitation molecular gas. Similar hyperfine anomalies are frequently seen in cold dark clouds where they are explained in this way (absorption in the envelope). The same effect is probably seen in $\mathrm{HCO}^{+}$. The $\mathrm{HCO}^{+}$line asymmetry apparently indicates the velocity shift of the foreground absorption. If attributed to the envelope, this shift indicates an expansion. In addition, $\mathrm{HCO}^{+}$perhaps traces a contribution from an outflow.

IRDC317.71-2 corresponds to the bright emission source in all mid-IR Spitzer/GLIMPSE bands. This, and all spectral features we discussed above indicates ongoing star-formation processes in IRDC317.71-2. A cross-correlation of the mid-IR data and near-IR 2MASS $K$-band images shows that the bright midIR source hides a young stellar cluster.

IRDC321.73 in IRDC321.73-1 (Fig. 2), although there is no emission in the GLIMPSE bands and only a very weak source at $24 \mu \mathrm{m}$, we detected clear $\mathrm{SiO}$ emission. This indicates the presence of a shock and probably an outflow activity in this region. The presence of the red $\mathrm{HCO}^{+}$shoulder could be an evidence of infall motion. The other two points in this cloud (IRDC321.732 and IRDC321.73-3) show a more complicated than average shape of $\mathrm{HCO}^{+}$. The rest of the lines, even optically thin like $\mathrm{N}_{2} \mathrm{H}^{+}$, also have non-Gaussian shapes. This can be explained by the presence of several kinematic components.

\section{Discussion}

Carey et al. (1998) and Pillai et al. (2006) mention a difference in the ammonia and formaldehyde abundances and hence a possible chemical difference between low-mass prestellar cores and IRDCs. To study the chemical conditions in IRDCs, Sakai et al. (2008) observed $\mathrm{N}_{2} \mathrm{H}^{+}(1-0), \mathrm{HC}_{3} \mathrm{~N}(5-4)$, $\mathrm{CCS}\left(4_{3}-3_{2}\right), \mathrm{NH}_{3}(1,1),(2,2),(3,3)$, and $\mathrm{CH}_{3} \mathrm{OH}(7-6)$ lines toward the massive clumps associated with IRDCs. They estimated the CCS and $\mathrm{N}_{2} \mathrm{H}^{+}$abundance ratio and used low-mass pre-stellar core abundances from Benson et al. (1998) for the comparison. The result of this analysis showed that IRDCs have lower $N(\mathrm{CCS}) / N\left(\mathrm{~N}_{2} \mathrm{H}^{+}\right)$ratio values and that therefore they can be chemically more evolved than low-mass pre-stellar cores. However, Sakai et al. (2008) used particular low-mass objects for the comparison and did not take into account the pre-stellar cores $\mathrm{L} 1512$ and L63, where $N(\mathrm{CCS}) / N\left(\mathrm{~N}_{2} \mathrm{H}^{+}\right)$ratio is low and agrees with values obtained for their IRDC sample.

Here we aim to determine if the molecular abundances in IRDCs are similar to the low-mass pre-stellar cores, or whether they show signatures of more evolved evolutionary stages. To answer this question we compare our IRDC abundances with the data available in the literature for low-mass starless clouds and more evolved high-mass objects. We analyze the dispersion of the particular abundance values and the difference between mean abundances for all available species. As an additional criterion, we compare the values of the $\mathrm{HNC} / \mathrm{HCN}$ abundance ratio for different types of objects.

\subsection{Abundance comparison with low-mass starless cores}

To make a comparison with low-mass cold clouds, we used the molecular abundances from Padovani et al. (2009), Tafalla et al. (2006), Jørgensen et al. (2004) and Ohishi et al. (1992) (Fig. 7 left panel). Comparing the dispersion of the abundance values we find that for some species, like $\mathrm{HC}_{3} \mathrm{~N}$, there is a large spread in abundance for the low-mass sample, but higher and lower values occur with regard to the IRDC values. For HNCO we compute abundances for low-mass starless cores using new results by Marcelino et al. (2009) and adopting the $\mathrm{H}_{2}$ column densities from Table 3 of Marcelino et al. (2010). That gives us quite low abundance values for B1, L1527-b, and L1544. However, cores like L183 and TMC-1 reach HNCO abundances that are overlapping with IRDC values and are just a factor of 2-3 lower than the IRDC average abundance. The same situation, in principle, is found for $\mathrm{HCO}^{+}$, where an overlap between the low-mass core and IRDC $\mathrm{HCO}^{+}$abundances exist for the objects L183 and TMC-1 (both attaining $8.0 \times 10^{-9}$ according to Ohishi et al. 1992). There is a tendency for higher abundances in IRDCs compared to low- mass cores for $\mathrm{N}_{2} \mathrm{H}^{+}$. However, here as well we found with $\mathrm{L} 1544$ a prominent example of a low-mass pre-stellar core with a rather high $\mathrm{N}_{2} \mathrm{H}^{+}$abundance of $5.0 \times 10^{-9}$ (Jørgensen et al. 2004).

The difference between mean abundances of IRDCs and lowmass objects is a factor of 2-3 for all species and around 5 in the extreme case of HNCO (see Table 3). Taking into account a large spread in abundances for every molecule within our IRDCs sample and systematic errors (see Sect. 3.4), these differences in average abundances between the low-mass pre-stellar cores and IRDCs cannot be used to claim a clear and unequivocal chemical distinction between the two groups of objects.

\subsection{Abundance comparison with high-mass protostellar objects}

The next class of objects that are interesting to compare with our results is the class of more evolved high-mass protostellar 

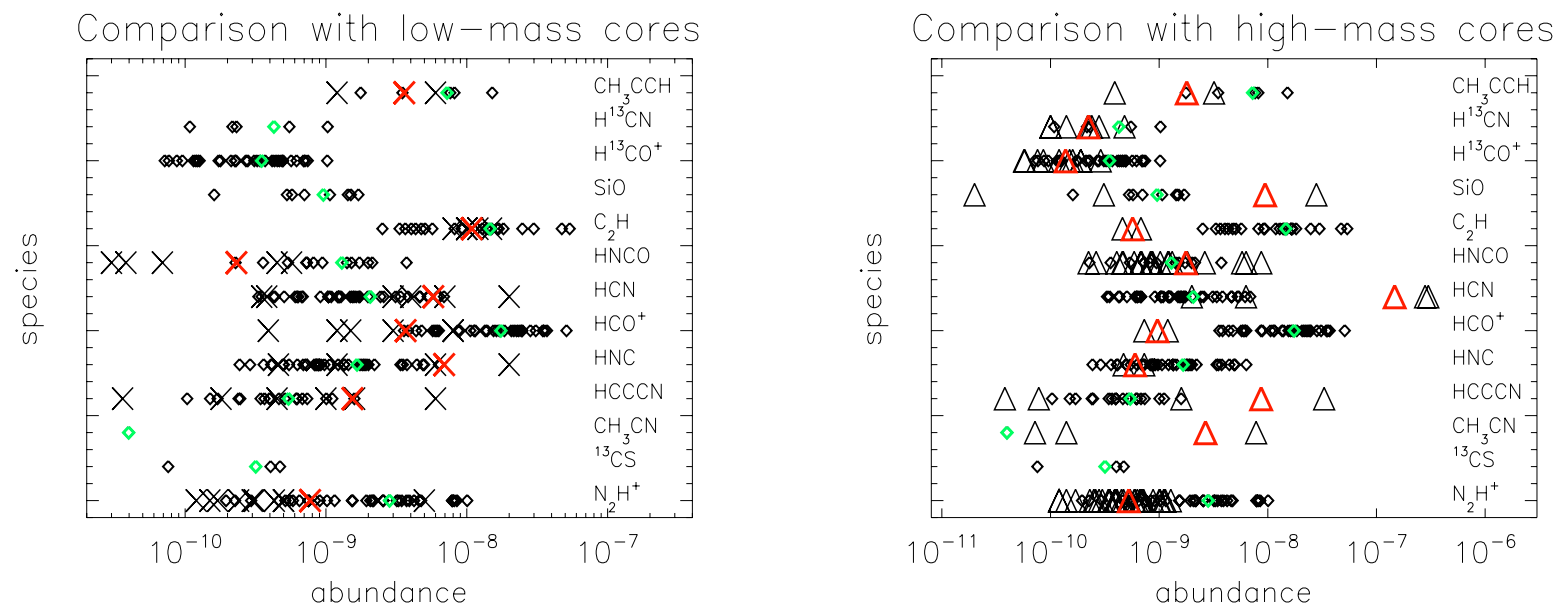

Fig. 7. (Left) Comparison of the molecular abundances of the IRDCs with low-mass pre-stellar cores from Padovani et al. (2009), Tafalla et al. (2006), Jørgensen et al. (2004) and Ohishi et al. (1992). Black diamonds indicate our IRDC abundances, green diamonds indicate mean values for IRDCs. Black crosses indicate low-mass starless cores, red crosses indicate mean values for low-mass starless cores. (Right) Comparison of the molecular abundances of the IRDCs with HMPOs from Blake et al. (1987), Helmich \& van Dishoeck (1997), Pirogov et al. (2003) and Zinchenko et al. (2000, 2009). Black diamonds indicate our IRDC abundances, green diamonds indicate mean values of the corresponding species for IRDCs. Black triangles indicate HMPOs, red triangles indicate mean values of the corresponding species for HMPOs. In both panels the upper limit abundance value for $\mathrm{CH}_{3} \mathrm{CN}$ for IRDCs was estimated from the average spectrum.

objects (HMPOs). HMPOs are bright at mid- and far-IR wavelengths and characterized by higher gas temperatures than IRDCs. It is assumed that these objects present one of the early stages of high-mass star formation, where the central protostar has a mass $>8 M_{\odot}$ and is still accreting (e.g. Sridharan et al. 2002; Beuther et al. 2007). For the comparison we use data from Blake et al. (1987), Helmich \& van Dishoeck (1997), Pirogov et al. (2003) and Zinchenko et al. (2000, 2009). HMPOs and IRDCs have different physical conditions (e.g. temperatures) and therefore expected to show different chemistry.

From our comparison we can see that high-mass protostellar objects show higher mean $\mathrm{HC}_{3} \mathrm{~N}$ and $\mathrm{HCN}$ abundances, because some HMPOs have extremely high abundances of these species. The mean abundance of $\mathrm{C}_{2} \mathrm{H}$ in HMPOs, on the contrary, is a factor of 20 lower. We did not detect $\mathrm{CH}_{3} \mathrm{CN}$ emission in any single IRDCs spectra. However, using the "average spectra" technique we reduce the noise level and find very weak $\mathrm{CH}_{3} \mathrm{CN}$ emission and estimate its upper abundance limit. This upper limit is lower compared with the HMPO abundances from single-dish studies by Helmich \& van Dishoeck (1997) and Blake et al. (1987) (see Fig. 7 right panel). Previous theoretical (Nomura \& Millar 2004) and observational (Beuther \& Sridharan 2007) studies of high-mass star-forming regions showed that a low amount of $\mathrm{CH}_{3} \mathrm{CN}$ is typical for the earliest evolutionary stages.

The average abundances of $\mathrm{HC}_{3} \mathrm{~N}, \mathrm{HCO}^{+}, \mathrm{HCN}$, and $\mathrm{C}_{2} \mathrm{H}$ for IRDCs and HMPOs differ by a factor of 16-75. These differences are significant even when considering all the included assumptions and uncertainties.

\subsection{HCN/HNC abundance ratio}

Another criterion that we use to determine the chemical status of IRDCs is the HCN/HNC abundance ratio. For the lowmass pre-stellar cores from Tafalla et al. (2006), Jørgensen et al. (2004) and Ohishi et al. (1992) this ratio is $\leq 1$. Values of the $\mathrm{HCN} / \mathrm{HNC}$ abundance ratio for HMPOs are higher and reach values of up to 13 (see Helmich \& van Dishoeck 1997; and
Blake et al. 1987) and are around 80 in the extreme regions like Orion (Goldsmith et al. 1986; Schilke et al. 1992). We find the $\mathrm{HCN} / \mathrm{HNC}$ abundance ratios for our IRDCs $\sim 1$. This value agrees with the theoretical predictions for the cold clouds (Sarrasin et al. 2010) and the values for the low-mass pre-stellar cores.

Considering the comparison between IRDCs, low-mass starless cores, and HMPOs and assuming that the HCN/HNC abundance ratio strongly depends on temperature and is enhanced in active star-forming cores (Goldsmith et al. 1986; Schilke et al. 1992), our results support the idea that IRDCs present rather low mass star-less core chemistry than HMPOs chemistry. However, the number of low-mass pre-stellar cores and HMPOs, that we used for the analysis may be not sufficient in a strict statistical sense. Therefore, to make a more solid statement about the evolutionary status of IRDCs and to be more confident, we need to extend the line samples, add sulfur-bearing species, and perform further studies, including chemical modeling.

Besides the comparison with low- and high-mass cloud abundances, we also compared molecular abundances of "quiescent", "middle" and "active" regions within our IRDC sample. In accordance with recent results by Battersby et al. (2010), we do not detect any significant difference among these three categories.

\section{Conclusions}

We presented $3 \mathrm{~mm}$ molecular line observations with the 22-m Mopra radio telescope. In total 13 molecular lines were observed for all IRDCs. The results of our study can be summarized as follows:

1. Using $\mathrm{H}_{2}$ column densities from the previous investigation, we estimated molecular abundances of all species. We showed that there is a tendency for the IRDCs to have molecular abundances similar to the low-mass pre-stellar cores rather than to the HMPOs abundances. However, the derived abundances come with uncertainties of around one order of magnitude. Furthermore, the comparison abundances for 
low-mass cores and HMPOs also can be affected by considerable uncertainties, especially when abundances have been computed in the literature by combining heterogeneous data sets (regarding beam sizes etc.). To make more solid statements about the evolutionary status of IRDCs therefore calls for subsequent systematical studies.

2. According to the classification of Chambers et al. (2009), we subdivided our clouds into "quiescent" and "active" and added a "middle" class. We found a trend for more evolved regions to have higher line widths and integrated intensities. However, we did not detect clear evidence of a different chemistry in these three groups.

3. A comparison of the line width and integrated intensities of the IRDCs and low-mass dark clouds shows values for IRDCs that are several times higher. Broader and more intense lines mean that in IRDCs we have more turbulent conditions compared with low-mass clouds.

4. We detect the $\mathrm{SiO}$ emission in some clouds and complicated shapes of the $\mathrm{HCO}^{+}$emission line profile in all IRDCs, which indicates infall and outflow motions and the beginning of star-formation activity, at least in some parts of the IRDCs.

5. The analysis of the two available $\mathrm{CH}_{3} \mathrm{C}_{2} \mathrm{H}$ excitation diagrams and detection of the very weak $\mathrm{CH}_{3} \mathrm{CN}\left(5_{0}-4_{0}\right)$ and $\mathrm{CH}_{3} \mathrm{CN}\left(5_{1}-4_{1}\right)$ lines on the "average spectra" indicate a warm gas component in some IRDCs. However, these warm regions are compact and cannot be resolved with single-dish observations.

Acknowledgements. The 22-m Mopra antenna is part of the Australia Telescope, which is funded by the Commonwealth of Australia for operations as a National Facility managed by CSIRO. The University of New South Wales Digital Filter Bank used for the observations with the Mopra Telescope was provided with support from the Australian Research Council. This research has made use of the NASA/ IPAC Infrared Science Archive, which is operated by the Jet Propulsion Laboratory, California Institute of Technology, under contract with the National Aeronautics and Space Administration. NASA's Astrophysics Data System was used to assess the literature given in the references.

We thank the anonymous referee and Malcolm Walmsley for valuable comments and suggestions that helped to improve this work. We also wish to thank Eric Herbst, Arjan Bik and Sarah Ragan for useful discussions and Sergej Koposov for the assistance in software installation.

I.Z. was partially supported by RFBR.

\section{References}

Afonso, J. M., Yun, J. L., \& Clemens, D. P. 1998, AJ, 115, 1111

Araya, E., Hofner, P., Kurtz, S., Bronfman, L., \& DeDeo, S. 2005, ApJS, 157, 279

Battersby, C., Bally, J., Jackson, J. M., et al. 2010, ApJ, 721, 222

Benjamin, R. A., Churchwell, E., Babler, B. L., et al. 2003, PASP, 115, 953

Benson, P. J., Caselli, P., \& Myers, P. C. 1998, ApJ, 506, 743

Bergin, E. A., Goldsmith, P. F., Snell, R. L., \& Ungerechts, H. 1994, ApJ, 431, 674

Bergin, E. A., Snell, R. L., \& Goldsmith, P. F. 1996, ApJ, 460, 343

Beuther, H., \& Henning, T. 2009, A\&A, 503, 859

Beuther, H., \& Sridharan, T. K. 2007, ApJ, 668, 348

Beuther, H., Churchwell, E. B., McKee, C. F., \& Tan, J. C. 2007, Protostars and Planets V, 165

Beuther, H., Semenov, D., Henning, T., \& Linz, H. 2008, ApJ, 675, L33

Blake, G. A., Sutton, E. C., Masson, C. R., \& Phillips, T. G. 1987, ApJ, 315, 621

Bronfman, L., Nyman, L., \& May, J. 1996, A\&AS, 115, 81

Carey, S. J., Clark, F. O., Egan, M. P., et al. 1998, ApJ, 508, 721

Caselli, P., Benson, P. J., Myers, P. C., \& Tafalla, M. 2002, ApJ, 572, 238

Chambers, E. T., Jackson, J. M., Rathborne, J. M., \& Simon, R. 2009, ApJS, 181, 360

Codella, C., Bachiller, R., Nisini, B., Saraceno, P., \& Testi, L. 2001, A\&A, 376, 271

Cyganowski, C. J., Whitney, B. A., Holden, E., et al. 2008, AJ, 136, 2391
De Buizer, J. M., \& Vacca, W. D. 2010, AJ, 140, 196

Egan, M. P., Shipman, R. F., Price, S. D., et al. 1998, ApJ, 494, L199 Fuller, G. A., Williams, S. J., \& Sridharan, T. K. 2005, A\&A, 442, 949

Gibson, D., Plume, R., Bergin, E., Ragan, S., \& Evans, N. 2009, ApJ, 705, 123

Goldsmith, P. F., Irvine, W. M., Hjalmarson, A., \& Ellder, J. 1986, ApJ, 310, 383

Helmich, F. P., \& van Dishoeck, E. F. 1997, A\&AS, 124, 205

Heyer, M. H., Carpenter, J. M., \& Snell, R. L. 2001, ApJ, 551, 852

Hofner, P., Wiesemeyer, H., \& Henning, T. 2001, ApJ, 549, 425

Jackson, J. M., Finn, S. C., Rathborne, J. M., Chambers, E. T., \& Simon, R. 2008, ApJ, 680, 349

Jones, P. A., Burton, M. G., Cunningham, M. R., et al. 2008, MNRAS, 386, 117 Jørgensen, J. K., Schöier, F. L., \& van Dishoeck, E. F. 2004, A\&A, 416, 603

Kalenskii, S. V., Promislov, V. G., Alakoz, A., Winnberg, A. V., \& Johansson,

L. E. B. 2000, A\&A, 354, 1036

Krumholz, M. R., \& McKee, C. F. 2008, Nature, 451, 1082

Ladd, N., Purcell, C., Wong, T., \& Robertson, S. 2005, PASA, 22, 62

Lee, C. W., Myers, P. C., \& Tafalla, M. 2001, ApJS, 136, 703

Lee, M., Stanimirović, S., Ott, J., et al. 2009, AJ, 138, 1101

Lo, N., Cunningham, M., Bains, I., Burton, M. G., \& Garay, G. 2007, MNRAS, 381, L30

Marcelino, N., Cernicharo, J., Tercero, B., \& Roueff, E. 2009, ApJ, 690, L27

Marcelino, N., Brünken, S., Cernicharo, J., et al. 2010, A\&A, 516, A105

Mardones, D., Myers, P. C., Tafalla, M., et al. 1997, ApJ, 489, 719

Müller, H. S. P., Thorwirth, S., Roth, D. A., \& Winnewisser, G. 2001, A\&A, 370, L49

Müller, H. S. P., Schlöder, F., Stutzki, J., \& Winnewisser, G. 2005, J. Molec. Struct., 742, 215

Myers, P. C., Mardones, D., Tafalla, M., Williams, J. P., \& Wilner, D. J. 1996, ApJ, 465, L133

Nomura, H., \& Millar, T. J. 2004, A\&A, 414, 409

Ohishi, M., Irvine, W. M., \& Kaifu, N. 1992, in Astrochemistry of Cosmic Phenomena, ed. P. D. Singh, IAU Symp., 150, 171

Padovani, M., Walmsley, C. M., Tafalla, M., Galli, D., \& Müller, H. S. P. 2009, A\&A, 505, 1199

Perault, M., Omont, A., Simon, G., et al. 1996, A\&A, 315, L165

Pillai, T., Wyrowski, F., Carey, S. J., \& Menten, K. M. 2006, A\&A, 450, 569

Pirogov, L., Zinchenko, I., Caselli, P., Johansson, L. E. B., \& Myers, P. C. 2003, A\&A, 405, 639

Purcell, C. R., Balasubramanyam, R., Burton, M. G., et al. 2006, MNRAS, 367, 553

Ragan, S. E., Bergin, E. A., Plume, R., et al. 2006, ApJS, 166, 567

Ragan, S. E., Bergin, E. A., \& Gutermuth, R. A. 2009, ApJ, 698, 324

Rathborne, J. M., Jackson, J. M., \& Simon, R. 2006, ApJ, 641, 389

Rawlings, J. M. C., Taylor, S. D., \& Williams, D. A. 2000, MNRAS, 313, 461

Rawlings, J. M. C., Redman, M. P., Keto, E., \& Williams, D. A. 2004, MNRAS, 351, 1054

Redman, M. P., Khanzadyan, T., Loughnane, R. M., \& Carolan, P. B. 2008, in Massive Star Formation: Observations Confront Theory, ed. H. Beuther, H. Linz, \& T. Henning, ASP Conf. Ser., 387, 38

Saito, H., Mizuno, N., Moriguchi, Y., et al. 2001, PASJ, 53, 1037

Sakai, T., Sakai, N., Kamegai, K., et al. 2008, ApJ, 678, 1049

Sakai, T., Sakai, N., Hirota, T., \& Yamamoto, S. 2010, ApJ, 714, 1658

Sarrasin, E., Abdallah, D. B., Wernli, M., et al. 2010, MNRAS, 404, 518

Schilke, P., Walmsley, C. M., Pineau Des Forets, G., et al. 1992, A\&A, 256, 595

Shirley, Y. L., Evans, II, N. J., Young, K. E., Knez, C., \& Jaffe, D. T. 2003, ApJS, 149,375

Simon, R., Jackson, J. M., Rathborne, J. M., \& Chambers, E. T. 2006, ApJ, 639, 227

Sridharan, T. K., Beuther, H., Schilke, P., Menten, K. M., \& Wyrowski, F. 2002, ApJ, 566, 931

Sridharan, T. K., Beuther, H., Saito, M., Wyrowski, F., \& Schilke, P. 2005, ApJ, 634, L57

Tafalla, M., Myers, P. C., Caselli, P., Walmsley, C. M., \& Comito, C. 2002, ApJ, 569,815

Tafalla, M., Santiago-García, J., Myers, P. C., et al. 2006, A\&A, 455, 577

Turner, B. E. 1971, ApJ, 163, L35

Vasyunina, T., Linz, H., Henning, T., et al. 2009, A\&A, 499, 149 (Paper I)

Viti, S. 2005, in Astrochemistry: Recent Successes and Current Challenges, ed.

D. C. Lis, G. A. Blake, \& E. Herbst, IAU Symp., 231, 67

Walsh, A. J., \& Burton, M. G. 2006, MNRAS, 365, 321

Zhang, Q., Sridharan, T. K., Hunter, T. R., et al. 2007, A\&A, 470, 269

Zinchenko, I., Henkel, C., \& Mao, R. Q. 2000, A\&A, 361, 1079

Zinchenko, I., Caselli, P., \& Pirogov, L. 2009, MNRAS, 395, 2234

Zinnecker, H., \& Yorke, H. W. 2007, ARA\&A, 45, 481

Pages 13 to 40 are available in the electronic edition of the journal at http: //www . aanda. org 
T. Vasyunina et al.: Chemistry in infrared dark clouds

\section{Appendix A: 3-color Spitzer/Glimpse images}
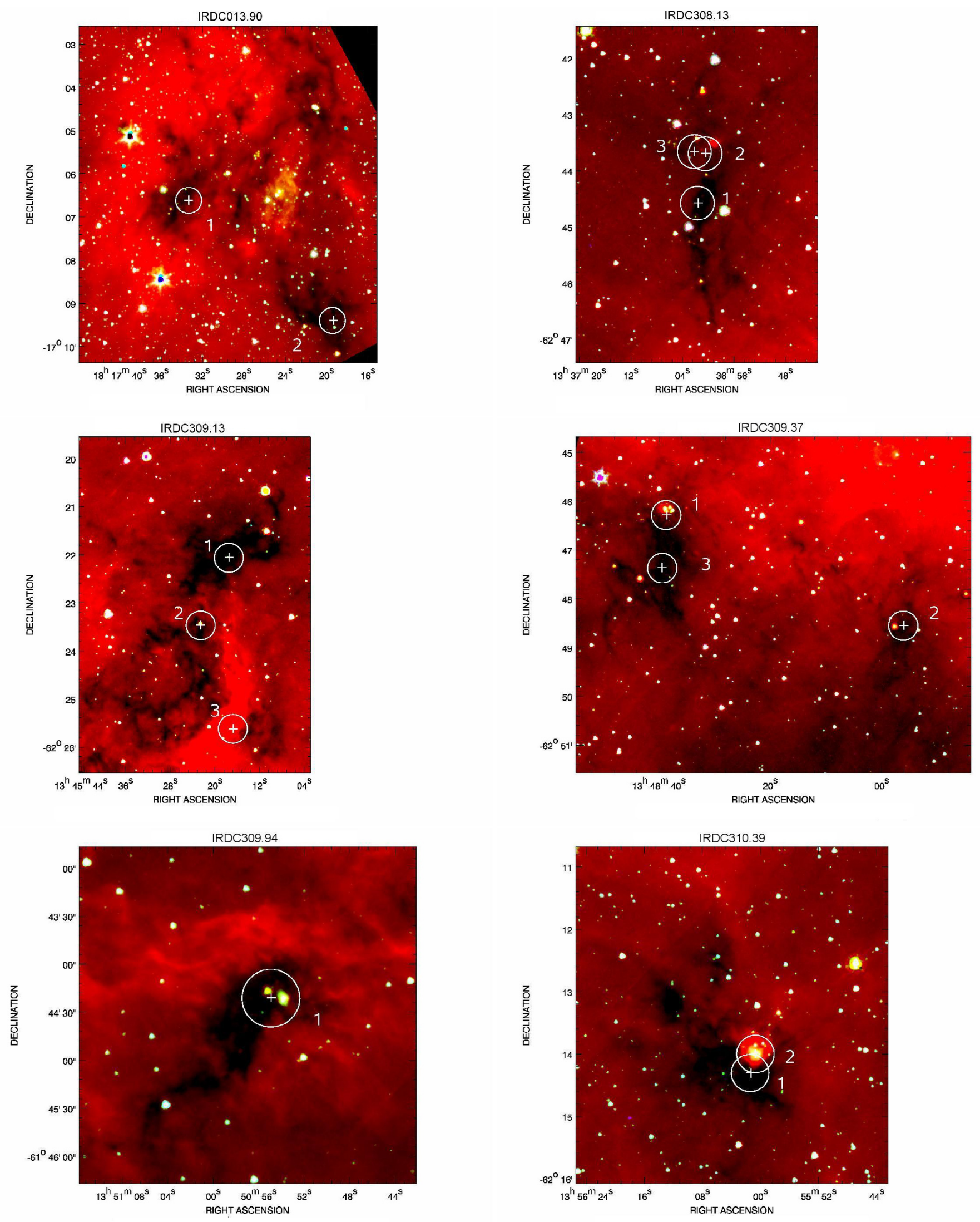

Fig. A.1. Three-color Spitzer/GLIMPSE image of the infrared dark cloud, where $3.6 \mu \mathrm{m}$ is blue, $4.5 \mu \mathrm{m}$ is green and $8 \mu \mathrm{m}$ is red. Circles mark observed positions and show the beam size. 

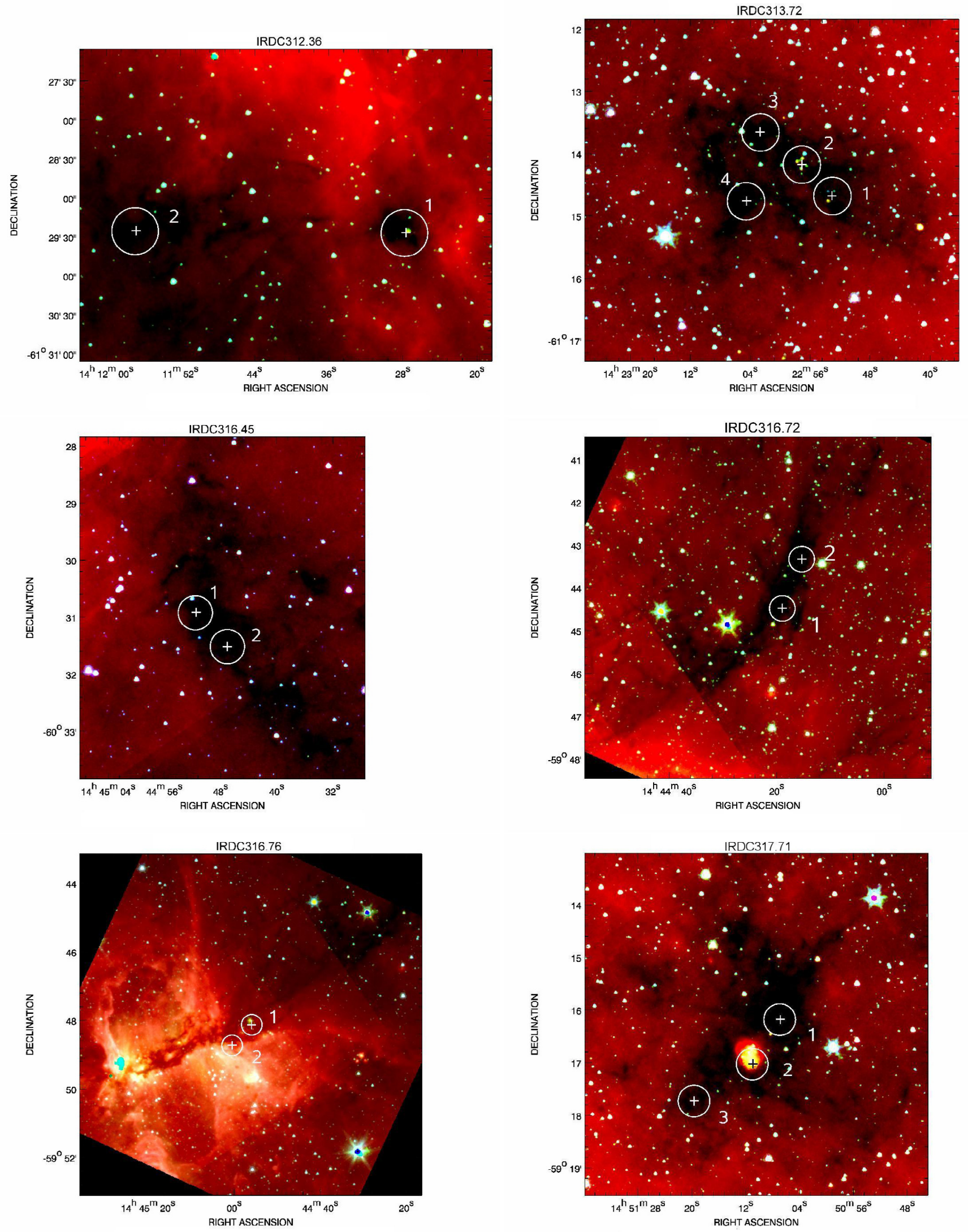

Fig. A.1. continued. 
T. Vasyunina et al.: Chemistry in infrared dark clouds
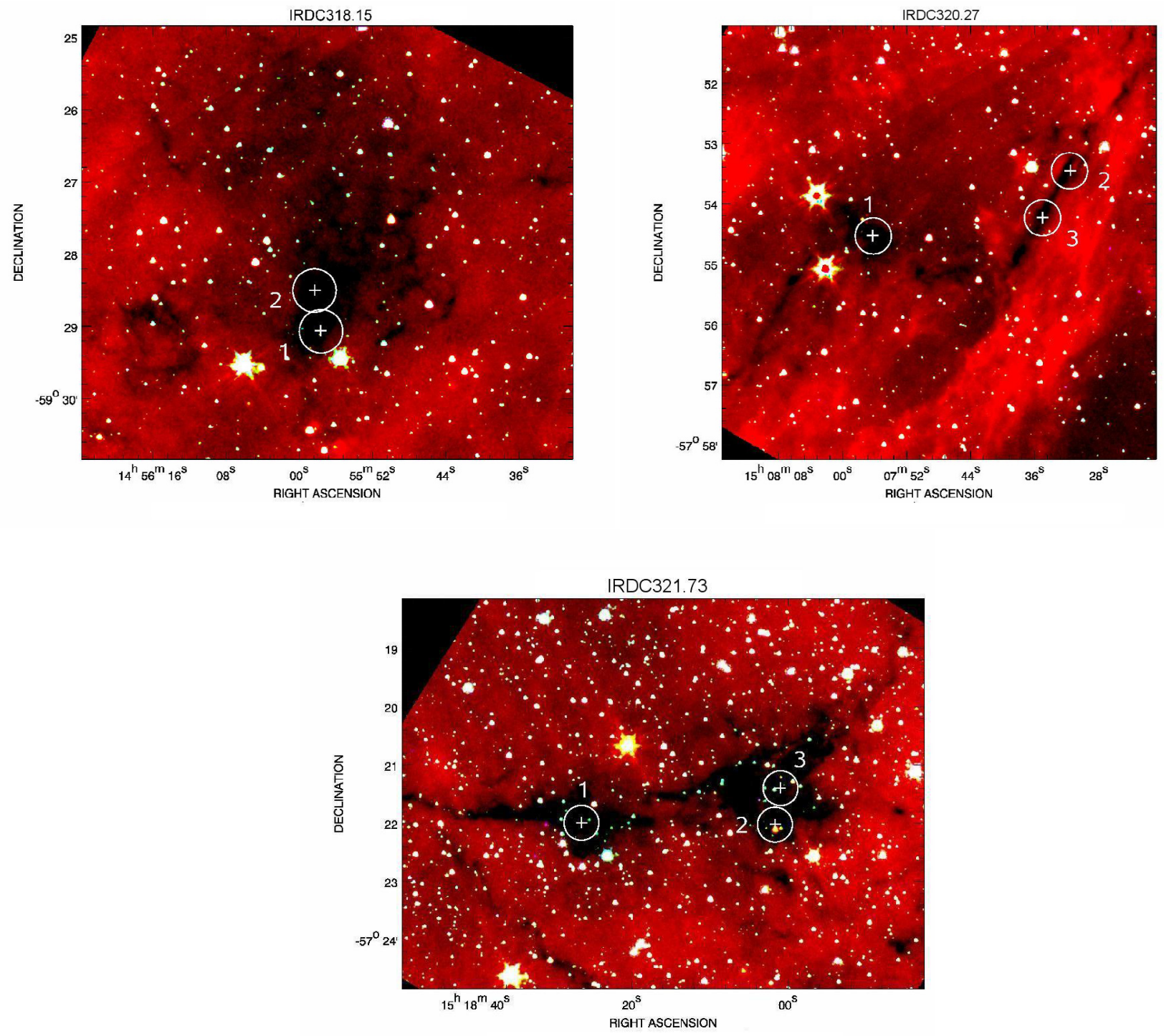

Fig. A.1. continued. 


\section{Appendix B: Spectra images}
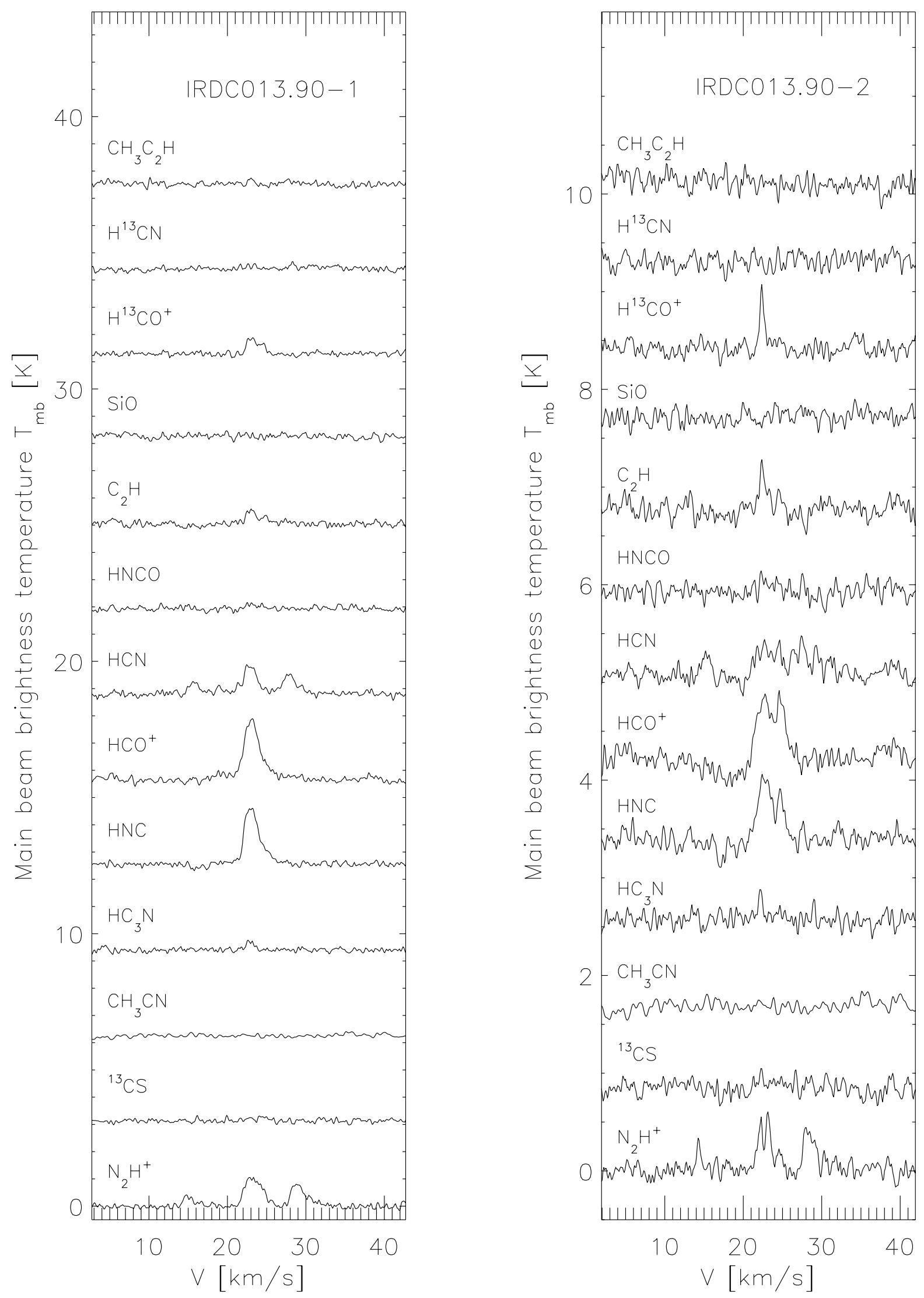

Fig. B.1. Observed lines. 
T. Vasyunina et al.: Chemistry in infrared dark clouds
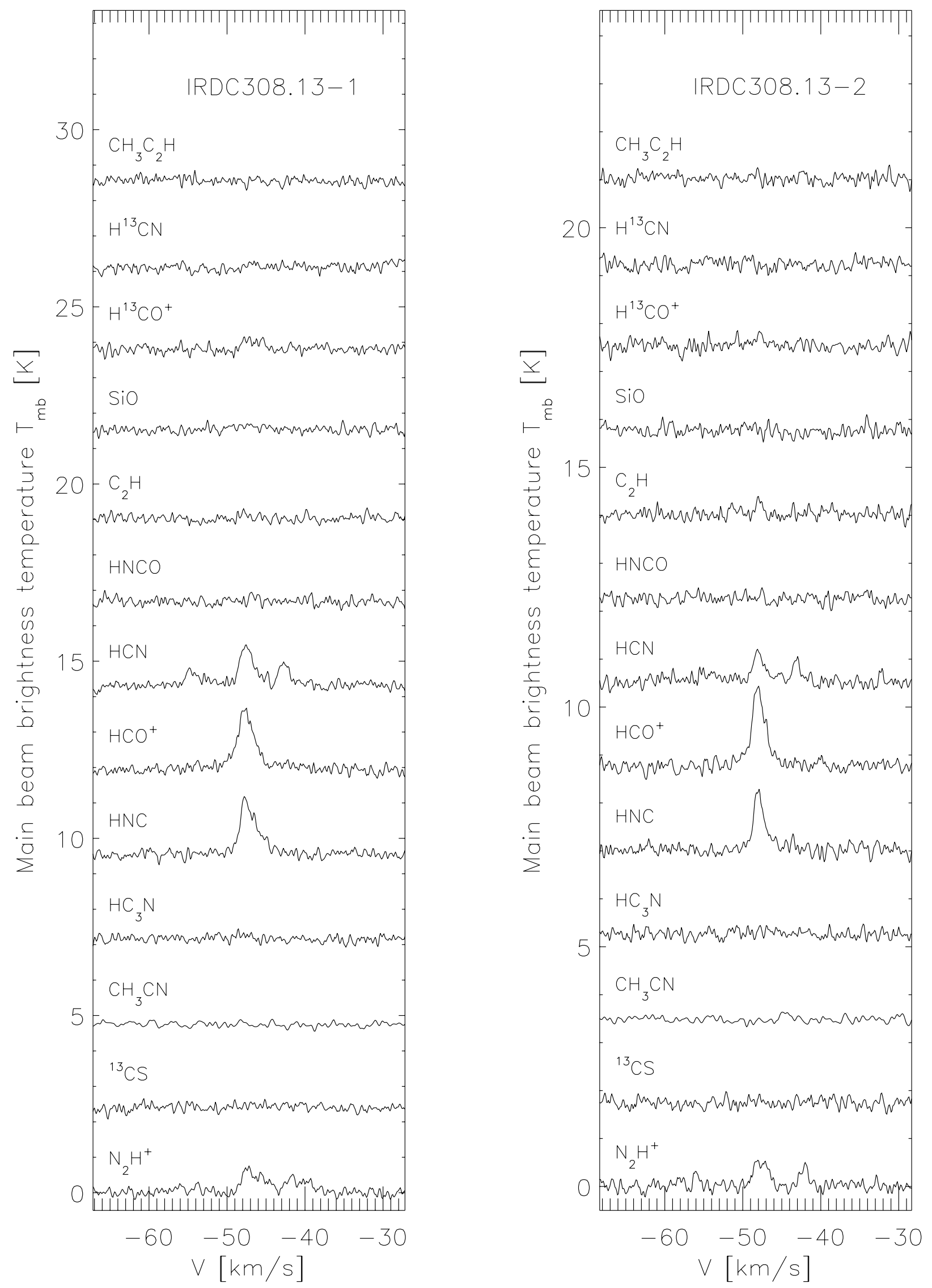

Fig. B.1. continued. 

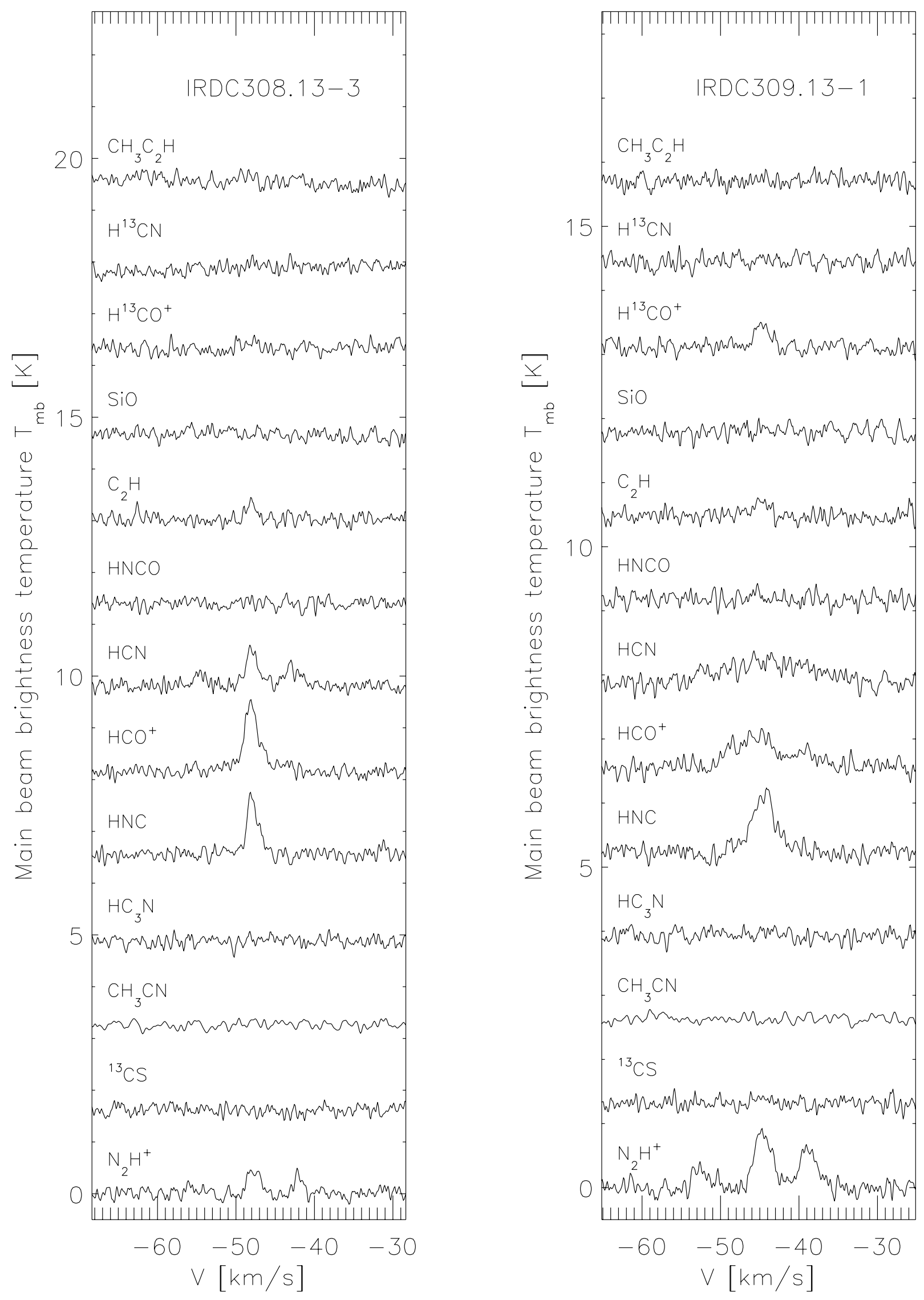

Fig. B.1. continued. 
T. Vasyunina et al.: Chemistry in infrared dark clouds
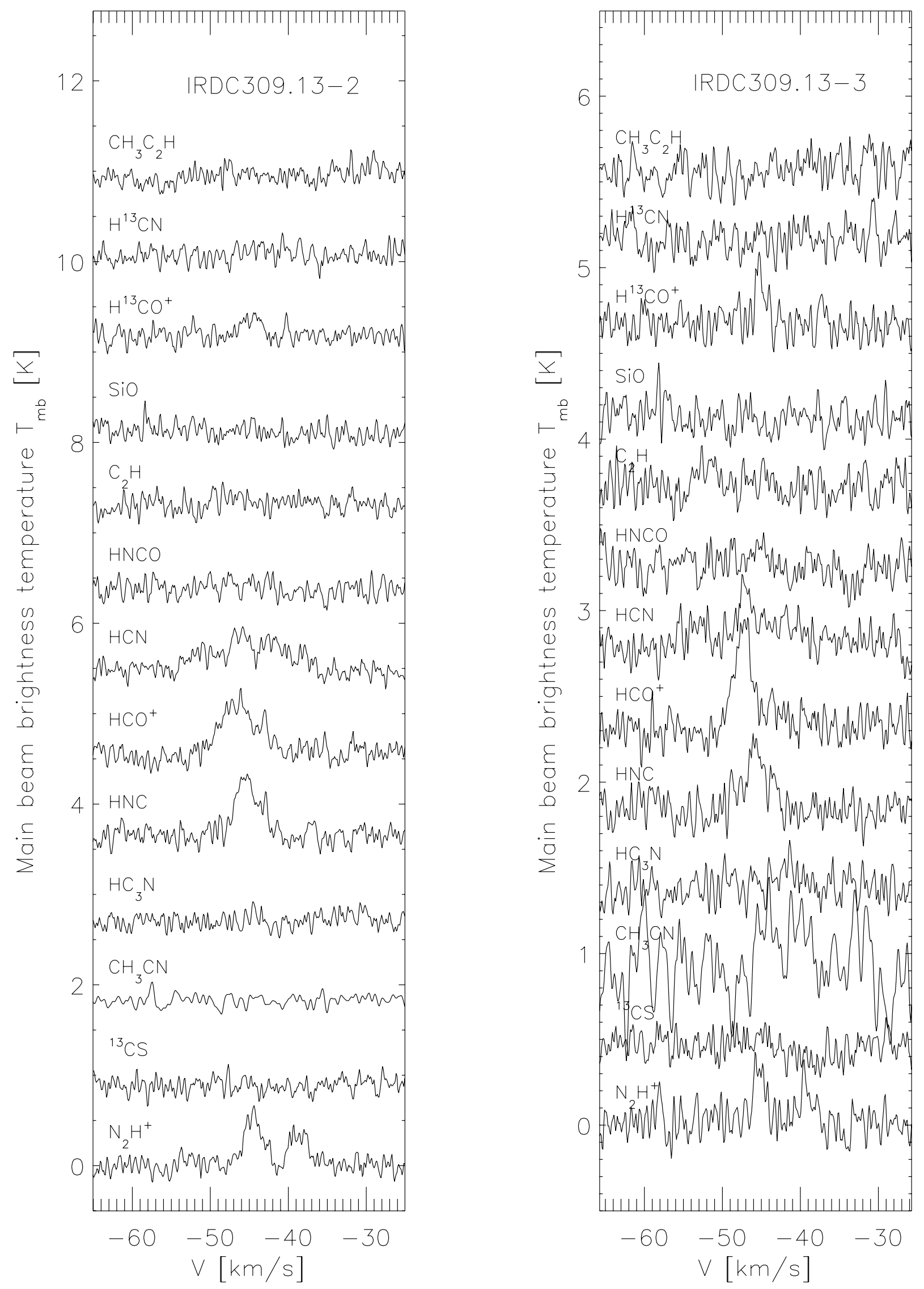

Fig. B.1. continued. 

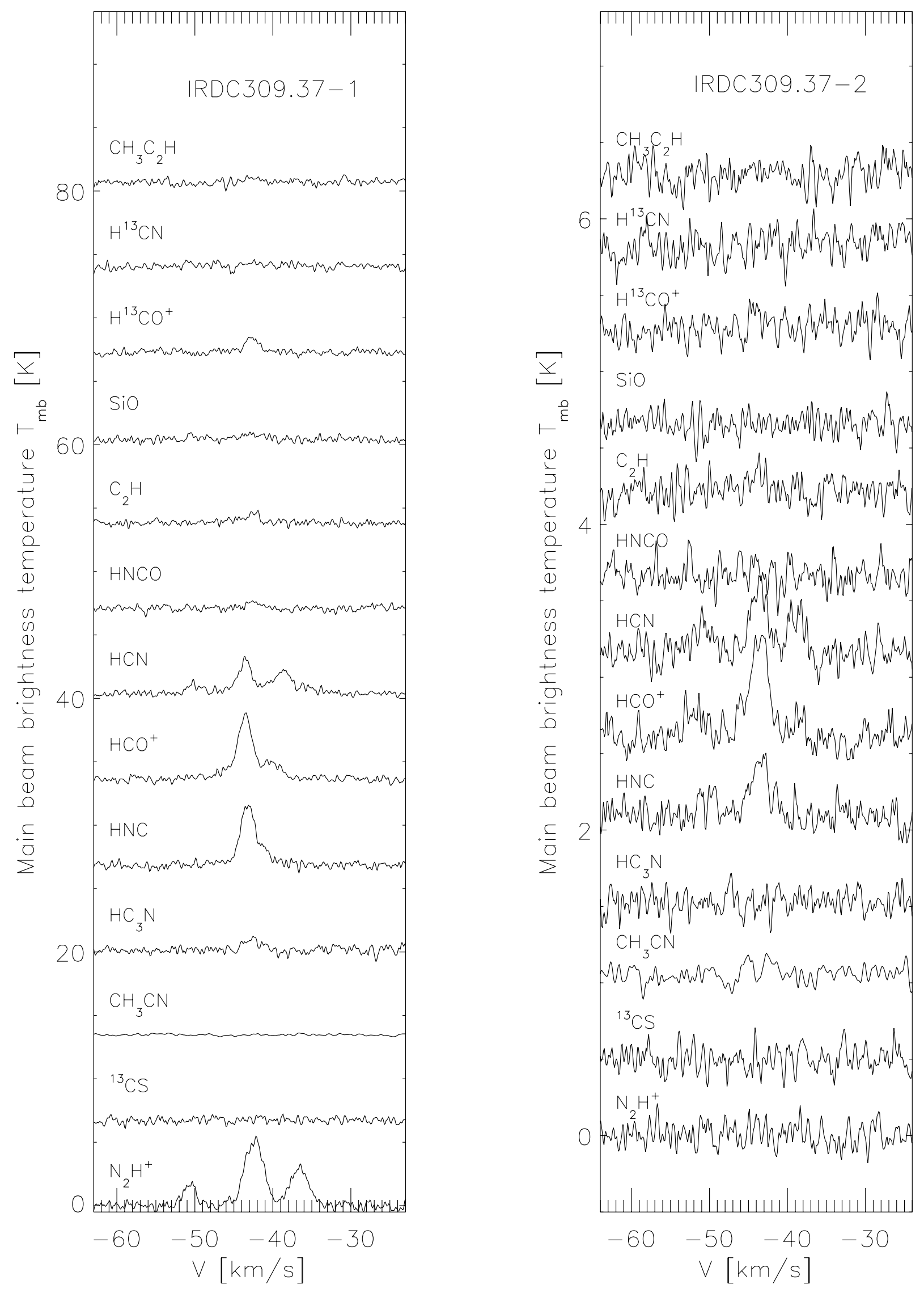

Fig. B.1. continued. 
T. Vasyunina et al.: Chemistry in infrared dark clouds
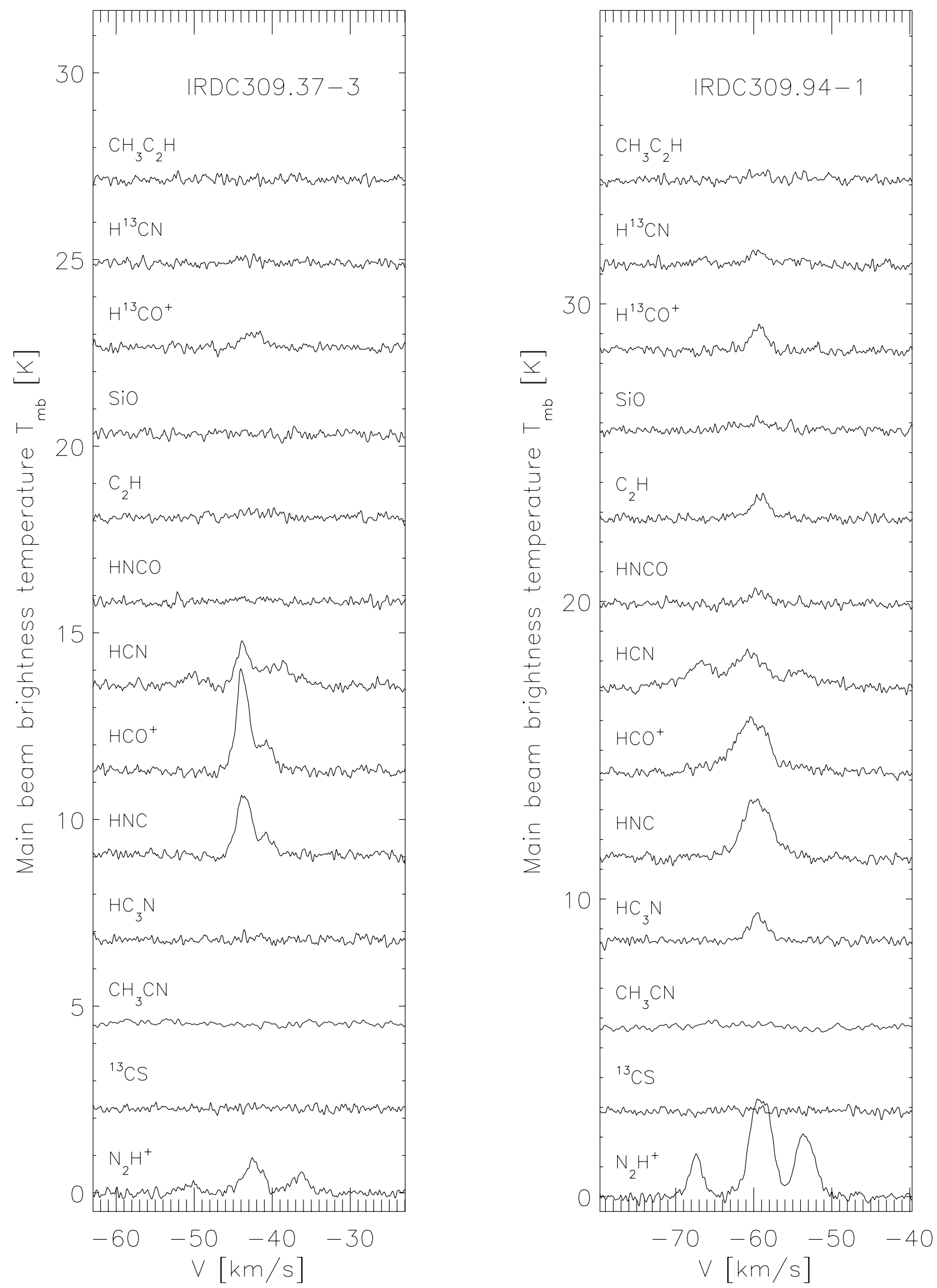

Fig. B.1. continued. 

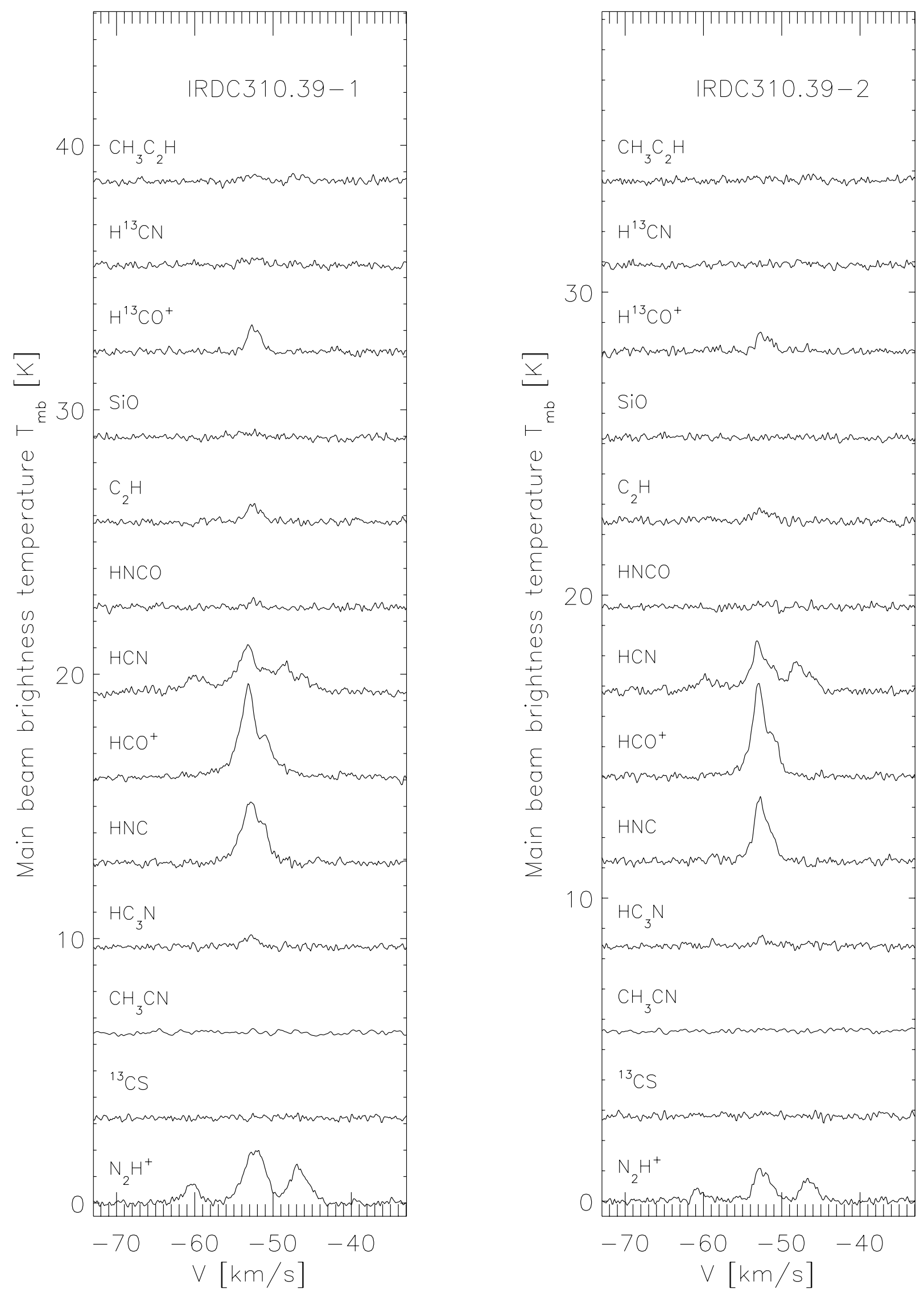

Fig. B.1. continued. 
T. Vasyunina et al.: Chemistry in infrared dark clouds
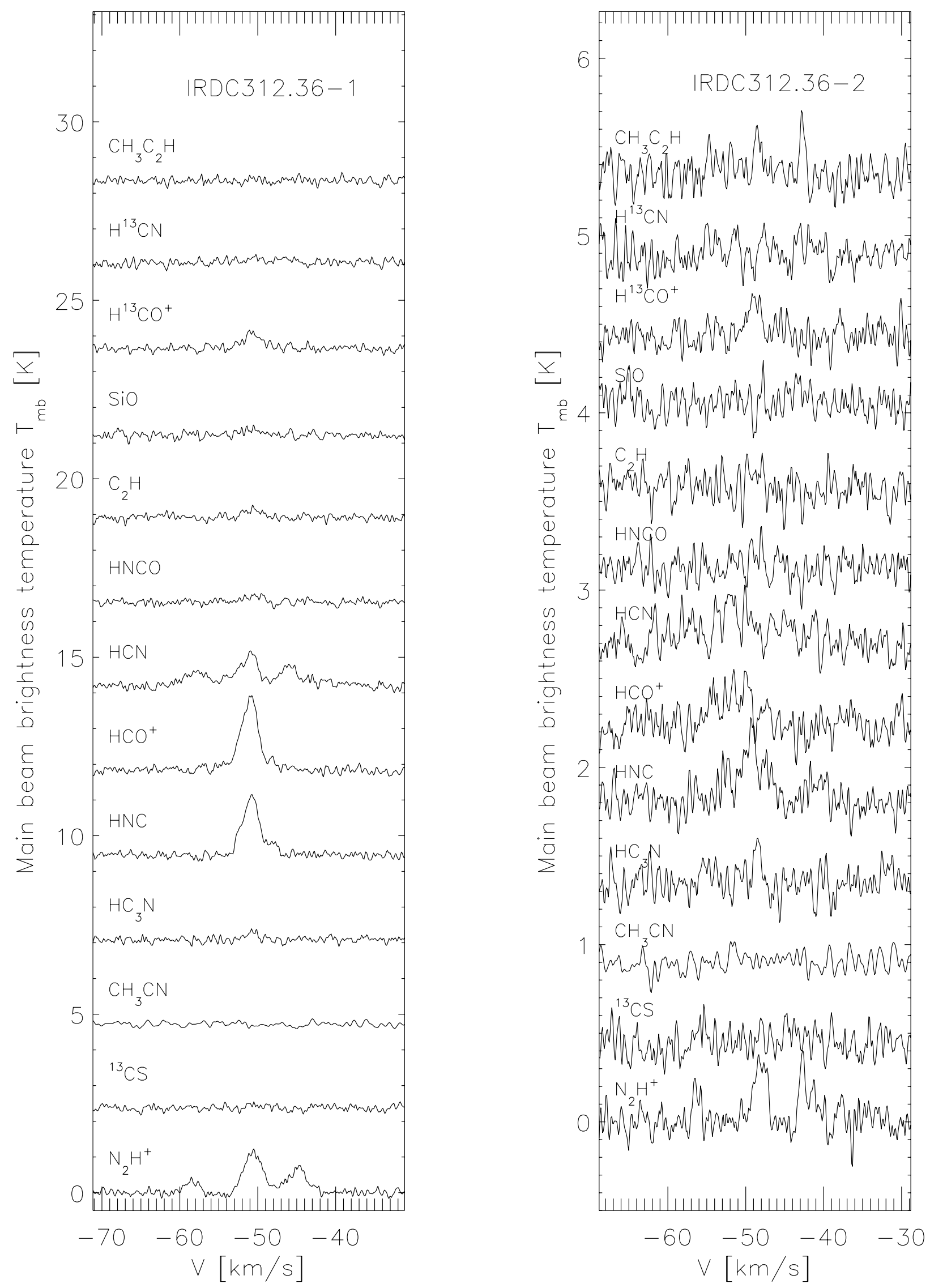

Fig. B.1. continued. 

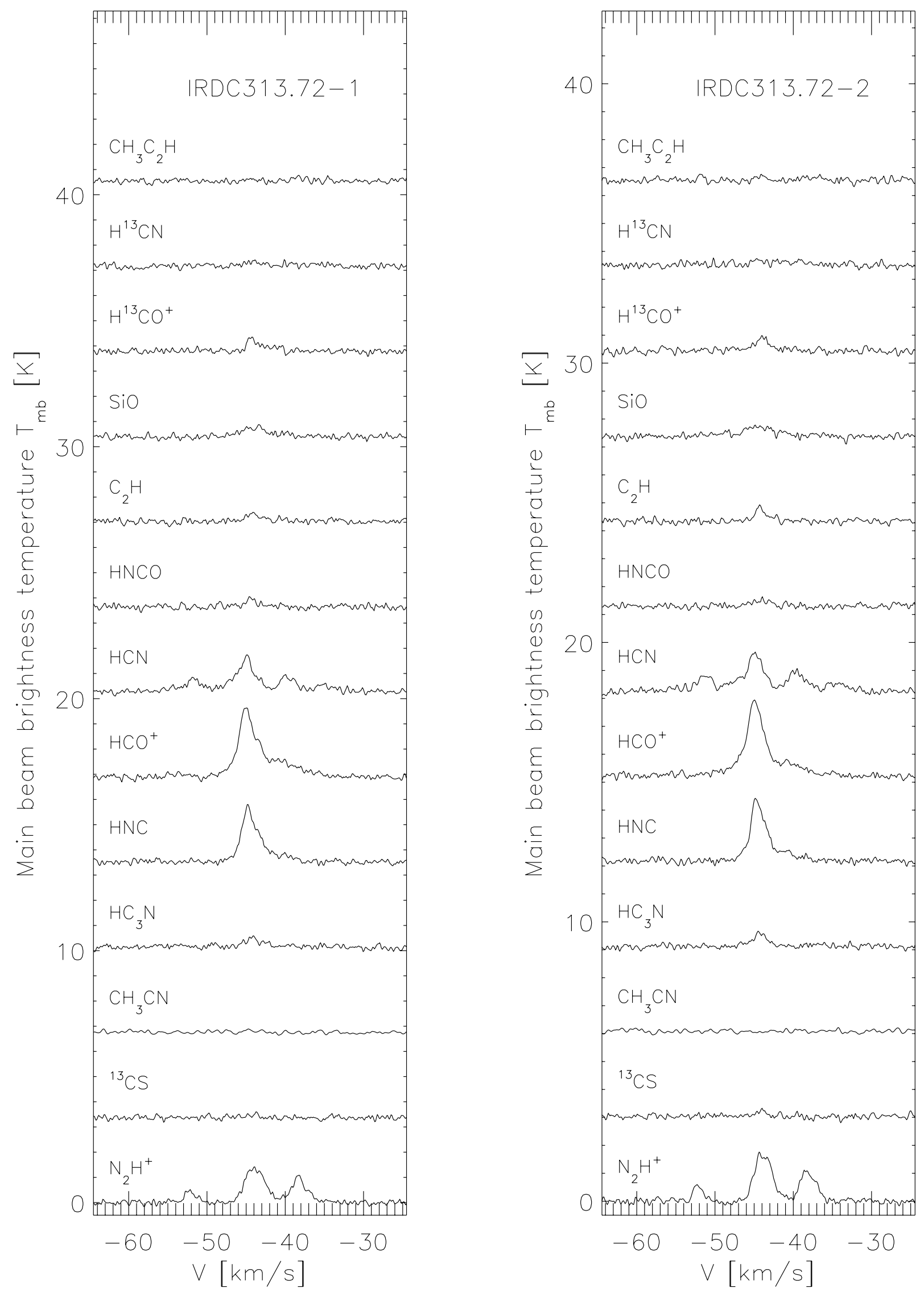

Fig. B.1. continued. 
T. Vasyunina et al.: Chemistry in infrared dark clouds
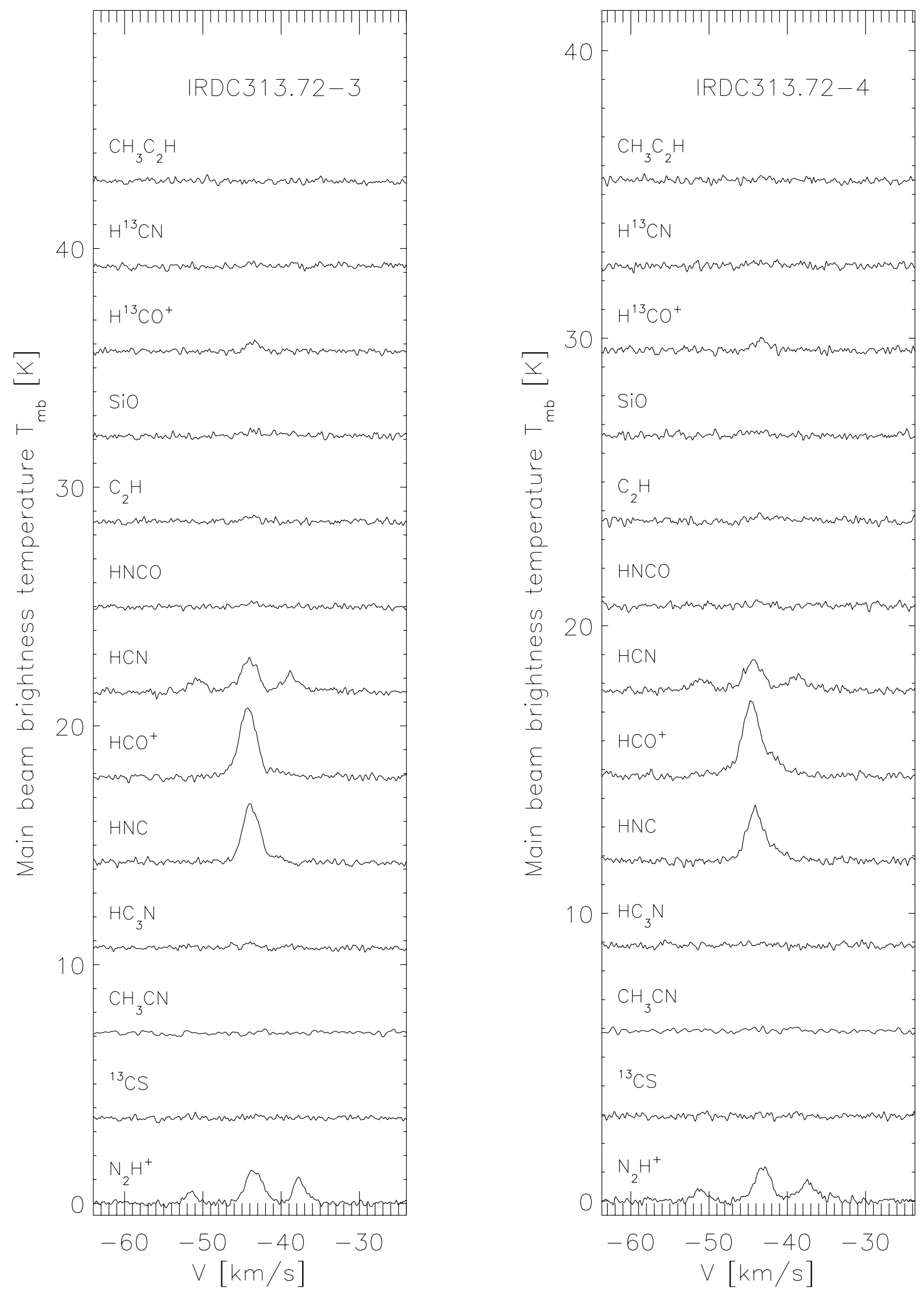

Fig. B.1. continued. 

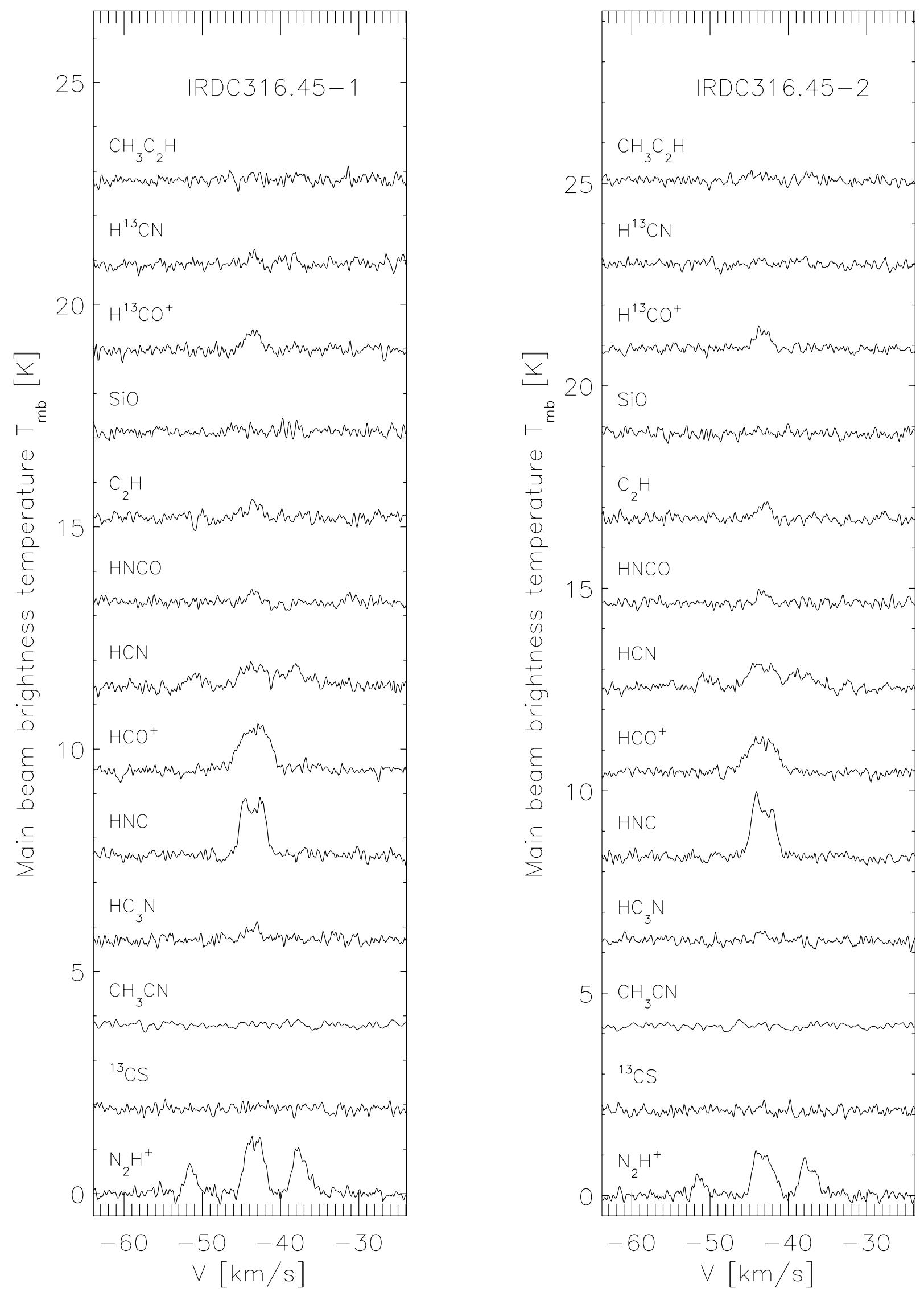

Fig. B.1. continued. 
T. Vasyunina et al.: Chemistry in infrared dark clouds
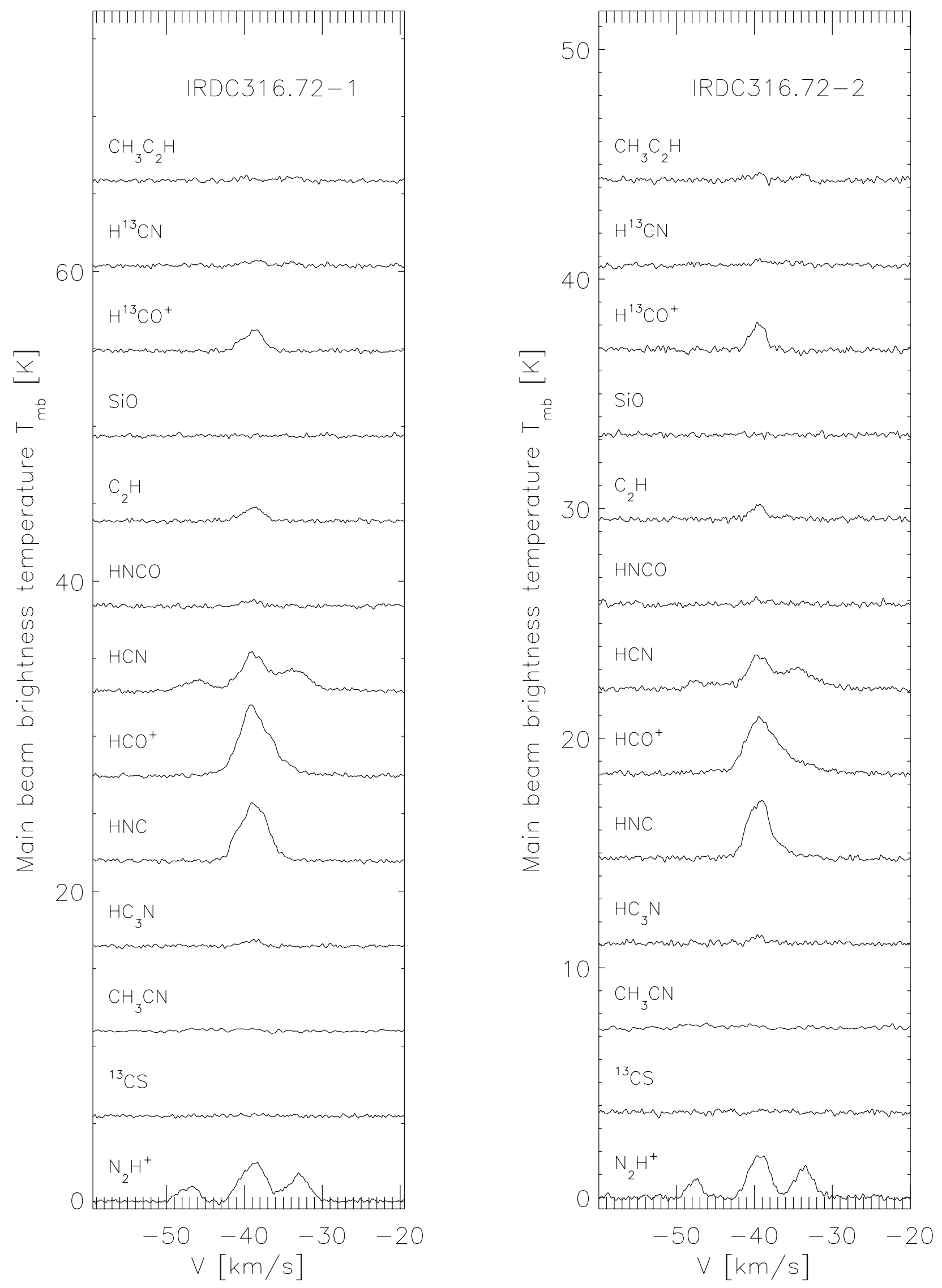

Fig. B.1. continued. 
A\&A 527, A88 (2011)
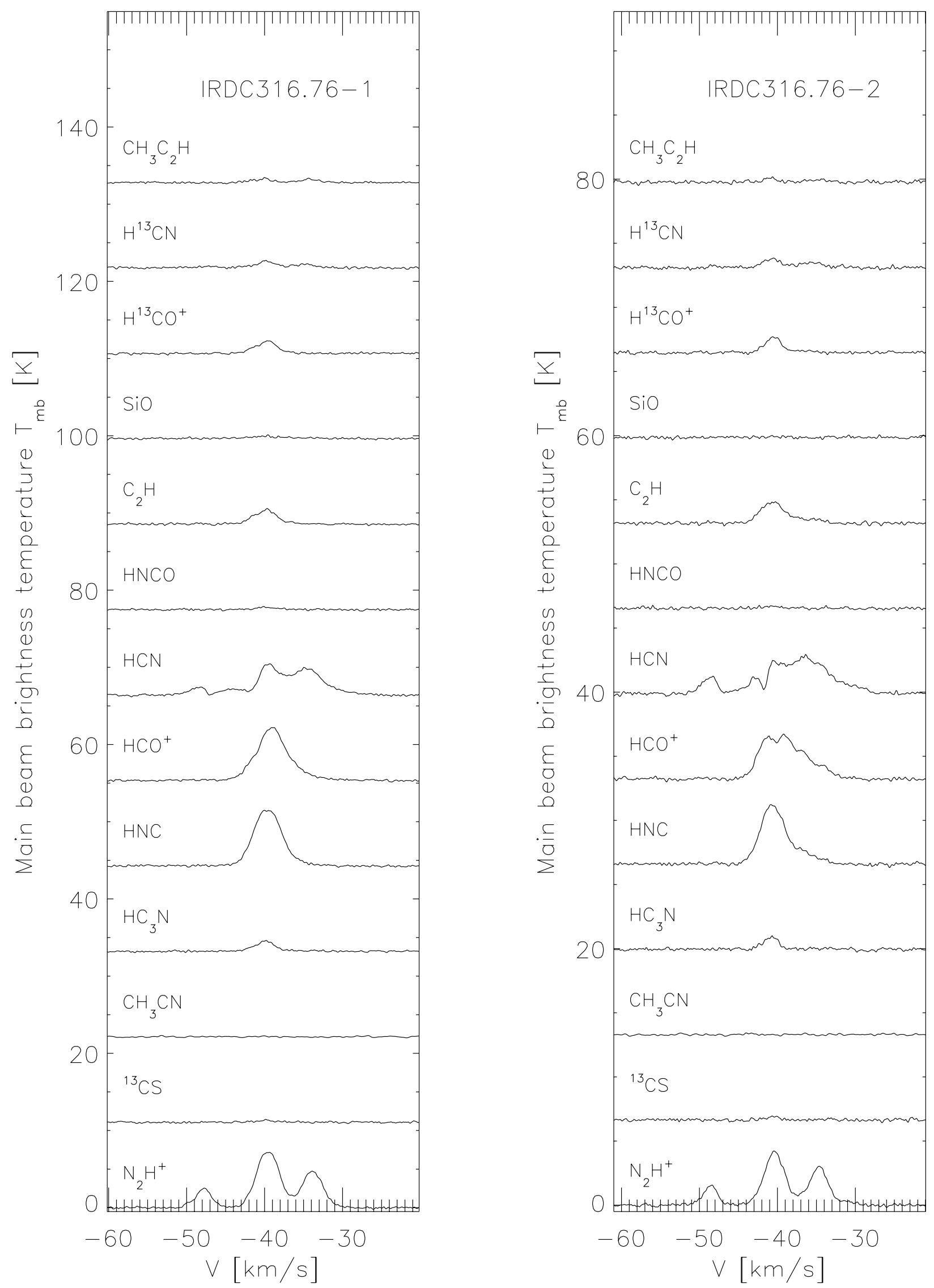

Fig. B.1. continued. 
T. Vasyunina et al.: Chemistry in infrared dark clouds
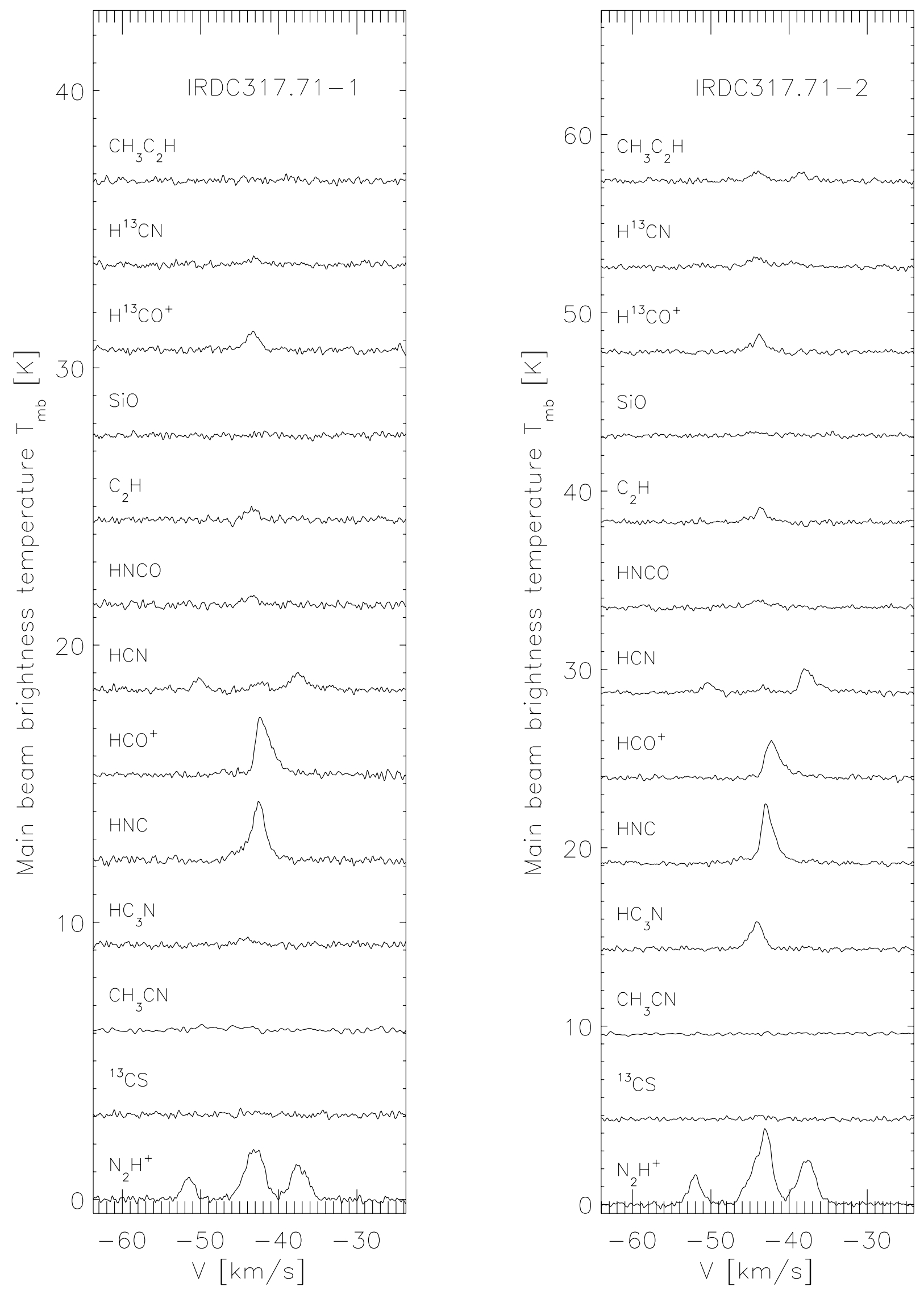

Fig. B.1. continued. 

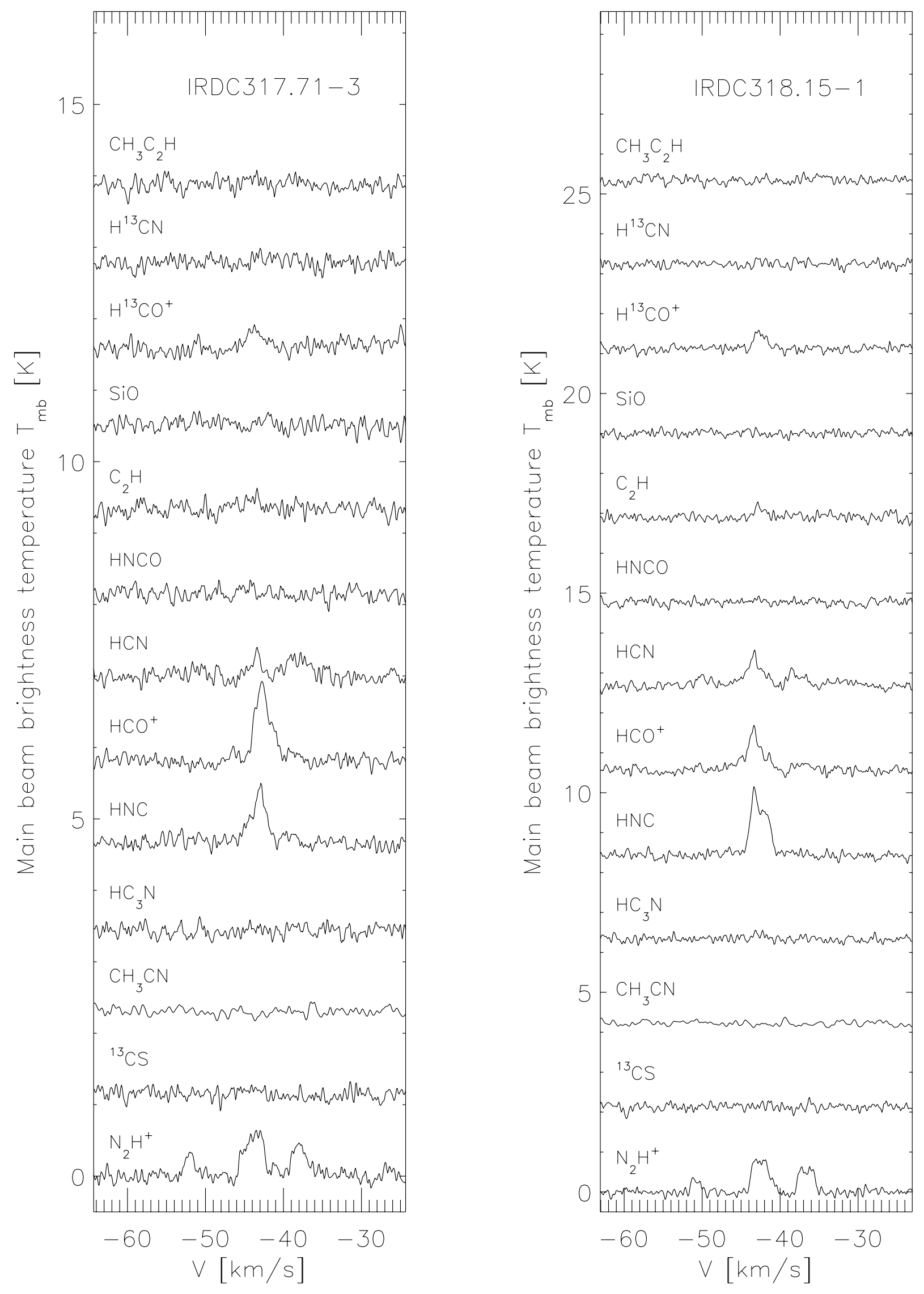

Fig. B.1. continued. 
T. Vasyunina et al.: Chemistry in infrared dark clouds
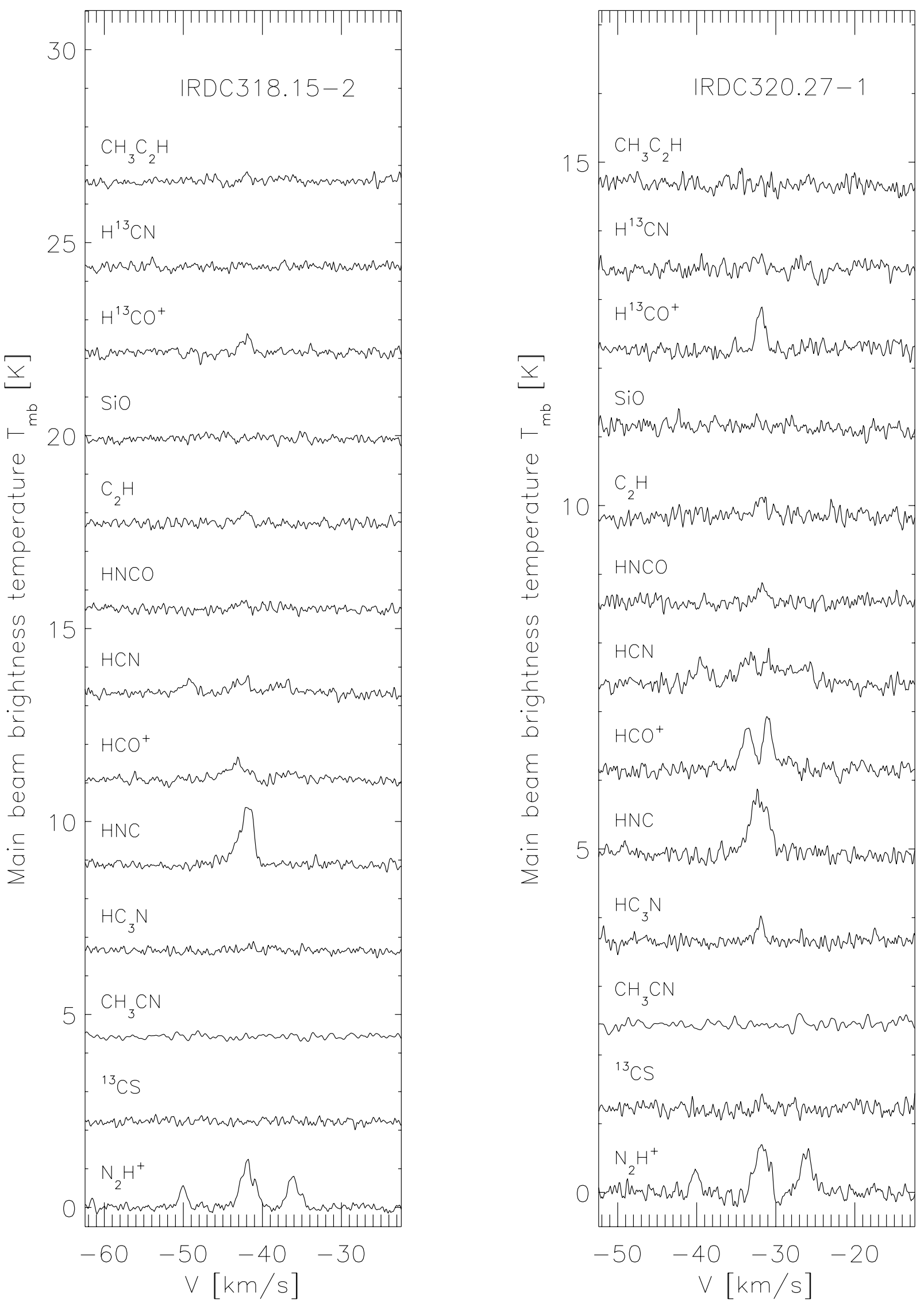

Fig. B.1. continued. 



Fig. B.1. continued. 
T. Vasyunina et al.: Chemistry in infrared dark clouds
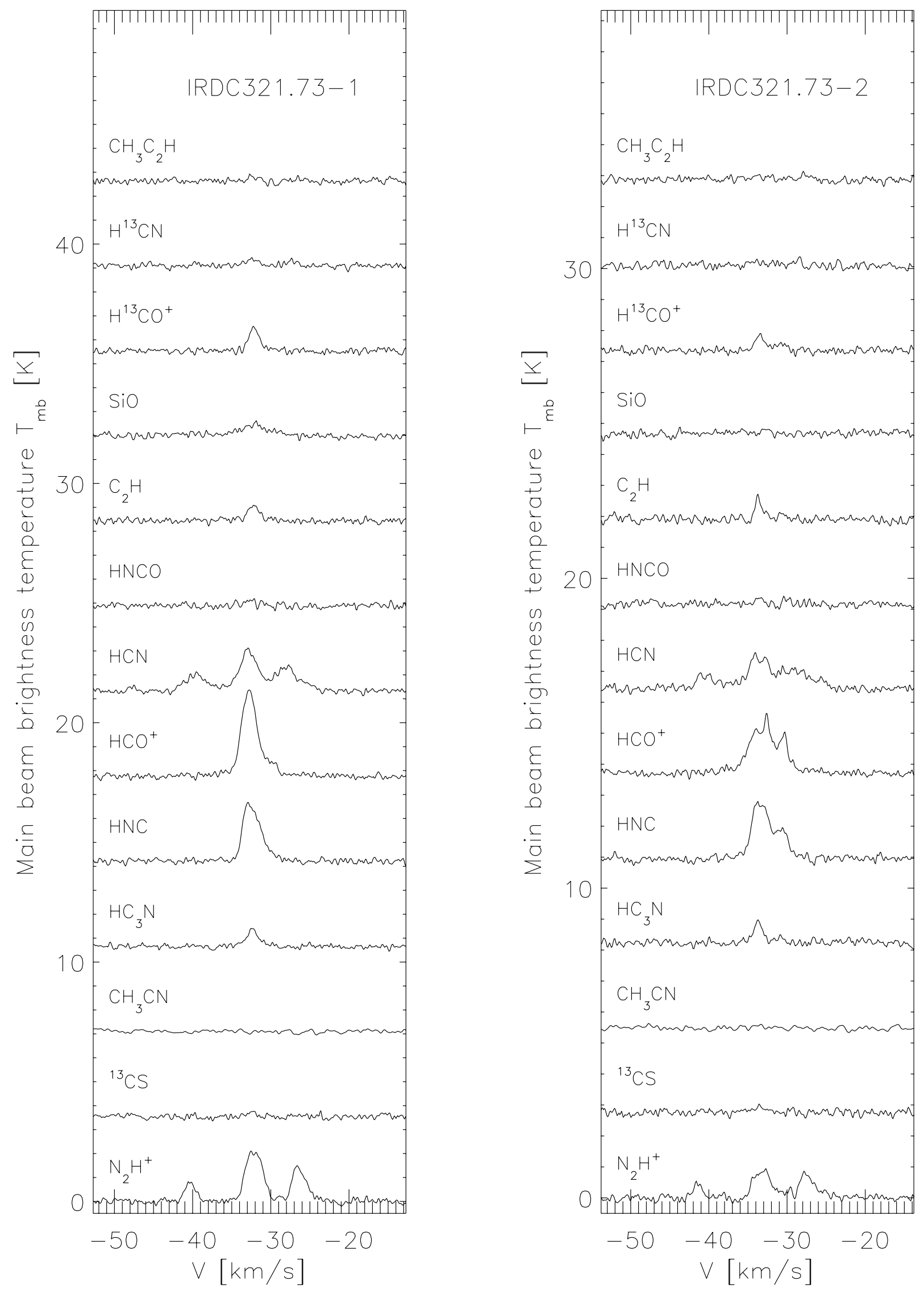

Fig. B.1. continued. 
A\&A 527, A88 (2011)

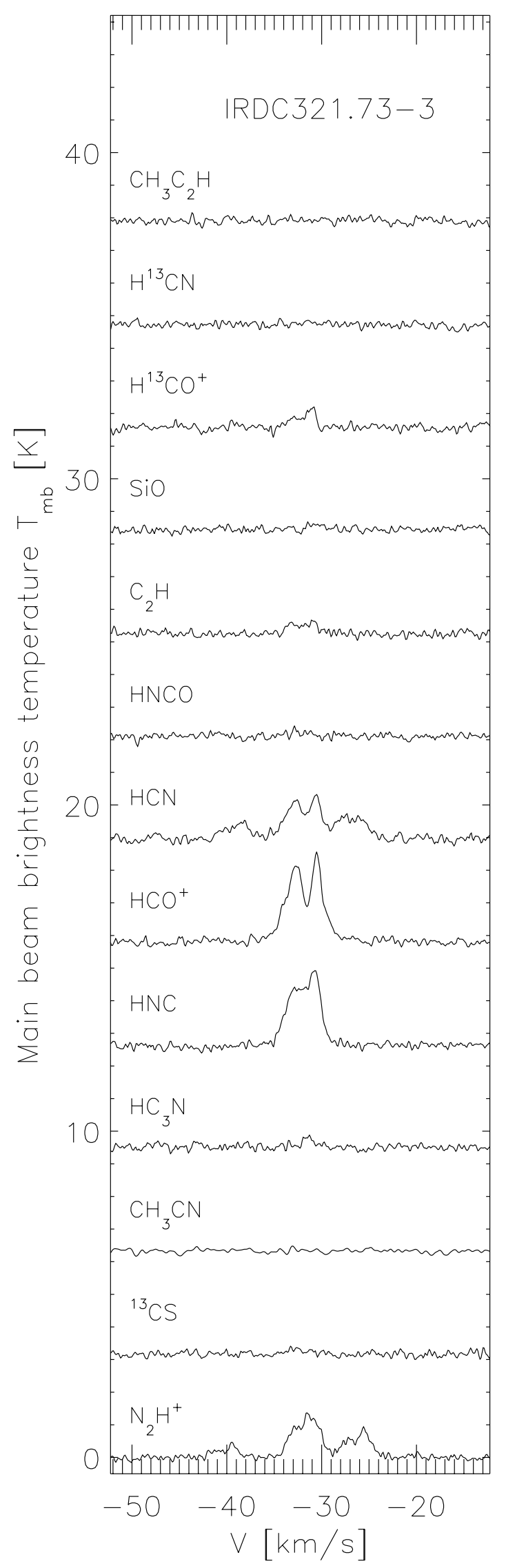

Fig. B.1. continued.

Page 34 of 40 
Appendix C: Line parameters

Table C.1. $\mathrm{N}_{2} \mathrm{H}^{+}$line parameters.

\begin{tabular}{|c|c|c|c|c|}
\hline Name & $\begin{array}{c}V_{\mathrm{lsr}} \\
\left(\mathrm{km} \mathrm{s}^{-1}\right)\end{array}$ & $\begin{array}{c}\Delta V \\
\left(\mathrm{~km} \mathrm{~s}^{-1}\right)\end{array}$ & $\tau_{\text {main }}$ & $\begin{array}{c}1 \sigma \mathrm{rms} \\
(\mathrm{K})\end{array}$ \\
\hline IRDC308.13-1 & $-46.8(0.09)$ & $3.0(0.24)$ & & 0.15 \\
\hline IRDC308.13-2 & $-47.9(0.04)$ & $0.7(0.11)$ & & 0.16 \\
\hline IRDC308.13-3 & $-47.8(0.03)$ & $0.9(0.08)$ & & 0.14 \\
\hline IRDC309.13-1 & $-44.7(0.04)$ & $1.8(0.16)$ & & 0.15 \\
\hline IRDC309.13-2 & $-44.6(0.06)$ & $2.3(0.12)$ & & 0.13 \\
\hline IRDC309.13-3 & $-45.3(0.04)$ & $0.9(0.13)$ & & 0.13 \\
\hline IRDC309.37-1 & $-42.5(0.02)$ & $2.4(0.00)$ & $0.2(0.007)$ & 0.42 \\
\hline IRDC309.37-3 & $-42.5(0.05)$ & $2.4(0.13)$ & & 0.13 \\
\hline IRDC310.39-1 & $-52.5(0.01)$ & $2.6(0.06)$ & $0.9(0.260)$ & 0.12 \\
\hline IRDC310.39-2 & $-52.5(0.03)$ & $1.8(0.12)$ & $1.6(0.744)$ & 0.13 \\
\hline IRDC312.36-1 & $-50.7(0.03)$ & $2.4(0.08)$ & & 0.12 \\
\hline IRDC312.36-2 & $-51.1(0.02)$ & $0.7(0.04)$ & & 0.11 \\
\hline IRDC313.72-1 & $-44.1(0.02)$ & $2.3(0.09)$ & & 0.12 \\
\hline IRDC313.72-2 & $-44.0(0.01)$ & $2.0(0.07)$ & & 0.12 \\
\hline IRDC313.72-3 & $-43.5(0.02)$ & $1.7(0.09)$ & & 0.12 \\
\hline IRDC313.72-4 & $-41.2(0.03)$ & $2.1(0.10)$ & & 0.12 \\
\hline IRDC316.72-1 & $-39.0(0.01)$ & $2.9(0.04)$ & $1.9(0.214)$ & 0.12 \\
\hline IRDC316.76-1 & $-39.7(0.00)$ & $2.8(0.00)$ & $0.8(0.015)$ & 0.12 \\
\hline IRDC316.72-2 & $-39.5(0.02)$ & $2.2(0.06)$ & $2.3(0.372)$ & 0.12 \\
\hline IRDC316.76-2 & $-40.5(0.01)$ & $2.5(0.03)$ & $1.4(0.166)$ & 0.12 \\
\hline IRDC317.71-1 & $-43.3(0.02)$ & $2.1(0.06)$ & $1.8(0.389)$ & 0.12 \\
\hline IRDC317.71-2 & $-43.6(0.00)$ & $2.4(0.00)$ & $0.9(0.129)$ & 0.12 \\
\hline IRDC317.71-3 & $-43.9(0.01)$ & $1.8(0.14)$ & $1.4(0.156)$ & 0.12 \\
\hline IRDC320.27-1 & $-31.9(0.04)$ & $1.0(0.09)$ & $4.3(1.918)$ & 0.13 \\
\hline IRDC320.27-2 & $-32.4(0.02)$ & $0.5(0.06)$ & & 0.13 \\
\hline IRDC320.27-3 & $-32.0(0.06)$ & $0.5(0.06)$ & & 0.07 \\
\hline IRDC321.73-1 & $-32.3(0.01)$ & $1.7(0.06)$ & $1.4(0.386)$ & 0.13 \\
\hline IRDC321.73-2 & $-33.3(0.03)$ & $1.5(0.13)$ & $4.5(1.172)$ & 0.12 \\
\hline IRDC321.73-3 & $-31.8(0.03)$ & $2.9(0.07)$ & & 0.12 \\
\hline IRDC013.90-1 & $23.1(0.03)$ & $1.6(0.10)$ & $1.8(0.635)$ & 0.12 \\
\hline IRDC013.90-2 & $22.5(0.03)$ & $1.3(0.10)$ & $2.6(0.967)$ & 0.11 \\
\hline IRDC316.45-1 & $-43.5(0.02)$ & $1.4(0.08)$ & $5.4(0.994)$ & 0.13 \\
\hline IRDC316.45-2 & $-43.4(0.03)$ & $1.6(0.10)$ & $4.3(1.063)$ & 0.14 \\
\hline IRDC318.15-1 & $-42.6(0.03)$ & $1.4(0.11)$ & $3.4(1.273)$ & 0.12 \\
\hline IRDC318.15-2 & $-42.0(0.02)$ & $1.0(0.08)$ & $2.7(1.093)$ & 0.12 \\
\hline IRDC309.94-1 & $-59.2(0.01)$ & $2.5(0.05)$ & $1.1(0.246)$ & 0.16 \\
\hline
\end{tabular}

Notes. Columns are name, LSR velocity, full linewidth at half maximum, main group optical depth, $1 \sigma$ rms values.

Table C.2. ${ }^{13} \mathrm{CS}$ line parameters.

\begin{tabular}{rccccc}
\hline \hline Name & $\begin{array}{c}\int T_{\mathrm{MB}} \mathrm{d} v \\
\left(\mathrm{~K} \mathrm{~km} \mathrm{~s}^{-1}\right)\end{array}$ & $\begin{array}{c}V_{\mathrm{lsr}} \\
\left(\mathrm{km} \mathrm{s}^{-1}\right)\end{array}$ & $\begin{array}{c}\Delta V \\
\left(\mathrm{~km} \mathrm{~s}^{-1}\right)\end{array}$ & $\begin{array}{c}T_{\mathrm{mb}} \\
(\mathrm{K})\end{array}$ & $\begin{array}{c}1 \sigma \mathrm{rms} \\
(\mathrm{K})\end{array}$ \\
\hline IRDC313.72-2 & $0.3(0.02)$ & $-44.1(0.1)$ & $1.3(0.2)$ & 0.2 & 0.06 \\
IRDC316.76-1 & $0.4(0.01)$ & $-39.8(0.1)$ & $1.0(0.0)$ & 0.4 & 0.06 \\
IRDC316.76-2 & $0.5(0.02)$ & $-40.6(0.1)$ & $1.9(0.2)$ & 0.3 & 0.06 \\
\hline
\end{tabular}

Notes. Columns are name, integrated area obtained from the Gaussian fits, LSR velocity, full linewidth at half maximum, main beam brightness temperature, $1 \sigma$ rms values. 
Table C.3. $\mathrm{HC}_{3} \mathrm{~N}$ line parameters.

\begin{tabular}{lccccc}
\hline \hline Name & $\begin{array}{c}\int T_{\mathrm{MB}} \mathrm{d} v \\
\left(\mathrm{~K} \mathrm{~km} \mathrm{~s}^{-1}\right)\end{array}$ & $\begin{array}{c}V_{\mathrm{lsr}} \\
\left(\mathrm{km} \mathrm{s}^{-1}\right)\end{array}$ & $\begin{array}{c}\Delta V \\
\left(\mathrm{~km} \mathrm{~s}^{-1}\right)\end{array}$ & $\begin{array}{c}T_{\mathrm{mb}} \\
(\mathrm{K})\end{array}$ & $\begin{array}{c}1 \sigma \mathrm{rms} \\
(\mathrm{K})\end{array}$ \\
\hline IRDC309.37-1 & $2.1(0.09)$ & $-42.6(0.10)$ & $2.3(0.2)$ & 0.8 & 0.42 \\
IRDC310.39-1 & $1.0(0.03)$ & $-52.8(0.06)$ & $2.4(0.1)$ & 0.4 & 0.13 \\
IRDC310.39-2 & $0.4(0.02)$ & $-52.5(0.07)$ & $1.5(0.2)$ & 0.2 & 0.13 \\
IRDC312.36-1 & $0.3(0.02)$ & $-50.7(0.09)$ & $1.4(0.2)$ & 0.2 & 0.12 \\
IRDC313.72-1 & $1.0(0.03)$ & $-44.0(0.09)$ & $3.0(0.2)$ & 0.3 & 0.13 \\
IRDC313.72-2 & $1.0(0.02)$ & $-44.2(0.05)$ & $2.1(0.1)$ & 0.4 & 0.12 \\
IRDC316.72-1 & $1.0(0.02)$ & $-39.0(0.07)$ & $2.6(0.1)$ & 0.3 & 0.12 \\
IRDC316.76-1 & $4.2(0.03)$ & $-39.9(0.02)$ & $3.0(0.1)$ & 1.2 & 0.12 \\
IRDC316.72-2 & $0.5(0.02)$ & $-39.5(0.06)$ & $1.8(0.1)$ & 0.3 & 0.12 \\
IRDC316.76-2 & $2.3(0.02)$ & $-40.8(0.02)$ & $2.2(0.0)$ & 0.9 & 0.12 \\
IRDC317.71-1 & $0.5(0.02)$ & $-44.2(0.12)$ & $2.3(0.2)$ & 0.2 & 0.13 \\
IRDC317.71-2 & $3.0(0.02)$ & $-44.1(0.01)$ & $2.0(0.0)$ & 1.4 & 0.13 \\
IRDC320.27-1 & $0.2(0.01)$ & $-31.9(0.05)$ & $0.8(0.1)$ & 0.3 & 0.13 \\
IRDC321.73-1 & $1.3(0.02)$ & $-32.3(0.03)$ & $1.8(0.1)$ & 0.6 & 0.13 \\
IRDC321.73-2 & $1.0(0.02)$ & $-33.7(0.03)$ & $1.4(0.1)$ & 0.6 & 0.13 \\
IRDC321.73-3 & $0.7(0.04)$ & $-31.3(0.10)$ & $2.4(0.5)$ & 0.2 & 0.13 \\
IRDC013.90-1 & $0.4(0.02)$ & $22.8(0.05)$ & $1.2(0.1)$ & 0.3 & 0.12 \\
IRDC316.45-1 & $0.6(0.03)$ & $-43.4(0.09)$ & $2.2(0.2)$ & 0.2 & 0.15 \\
IRDC309.94-1 & $2.7(0.04)$ & $-59.5(0.04)$ & $2.9(0.1)$ & 0.8 & 0.16 \\
\hline
\end{tabular}

Notes. Columns are name, integrated area obtained from the Gaussian fits, LSR velocity, full linewidth at half maximum, main beam brightness temperature, $1 \sigma$ rms values. 
Table C.4. HNC line parameters.

\begin{tabular}{|c|c|c|c|c|c|}
\hline Name & $\int_{\left(\mathrm{K} \mathrm{km} \mathrm{s}^{-1}\right)} T_{\mathrm{MB}} \mathrm{d} v$ & $\begin{array}{c}V_{\mathrm{lsr}} \\
\left(\mathrm{km} \mathrm{s}^{-1}\right)\end{array}$ & $\begin{array}{c}\Delta V \\
\left(\mathrm{~km} \mathrm{~s}^{-1}\right)\end{array}$ & $\begin{array}{l}T_{\mathrm{mb}} \\
(\mathrm{K})\end{array}$ & $\begin{array}{c}1 \sigma \mathrm{rms} \\
(\mathrm{K})\end{array}$ \\
\hline IRDC308.13-1 & $3.5(0.02)$ & $-47.3(0.02)$ & $2.1(0.00)$ & 1.5 & 0.16 \\
\hline IRDC308.13-2 & $2.1(0.03)$ & $-47.9(0.02)$ & $1.7(0.07)$ & 1.1 & 0.17 \\
\hline IRDC308.13-3 & $2.1(0.03)$ & $-47.8(0.02)$ & $1.8(0.08)$ & 1.0 & 0.16 \\
\hline IRDC309.13-1 & $3.2(0.04)$ & $-44.4(0.04)$ & $3.4(0.10)$ & 0.8 & 0.15 \\
\hline IRDC309.13-2 & $2.4(0.04)$ & $-45.2(0.06)$ & $3.7(0.15)$ & 0.6 & 0.14 \\
\hline IRDC309.13-3 & $1.1(0.05)$ & $-45.6(0.15)$ & $3.4(0.40)$ & 0.3 & 0.16 \\
\hline IRDC309.37-1 & $12.2(0.10)$ & $-43.1(0.02)$ & $2.5(0.05)$ & 4.4 & 0.41 \\
\hline IRDC309.37-2 & $1.0(0.03)$ & $-43.5(0.08)$ & $2.7(0.20)$ & 0.3 & 0.13 \\
\hline IRDC309.37-3 & $4.3(0.03)$ & $-43.4(0.02)$ & $2.7(0.06)$ & 1.5 & 0.13 \\
\hline IRDC310.39-1 & $7.9(0.03)$ & $-52.6(0.01)$ & $3.4(0.03)$ & 2.1 & 0.13 \\
\hline IRDC310.39-2 & $5.0(0.02)$ & $-52.5(0.01)$ & $2.5(0.03)$ & 1.8 & 0.13 \\
\hline IRDC312.36-1 & $4.5(0.03)$ & $-50.8(0.01)$ & $2.7(0.04)$ & 1.5 & 0.13 \\
\hline IRDC312.36-2 & $1.6(0.04)$ & $-49.4(0.13)$ & $5.3(0.36)$ & 0.2 & 0.12 \\
\hline IRDC313.72-1 & $4.7(0.02)$ & $-44.5(0.01)$ & $2.0(0.00)$ & 2.2 & 0.12 \\
\hline IRDC313.72-2 & $5.5(0.03)$ & $-44.4(0.01)$ & $2.5(0.03)$ & 2.0 & 0.12 \\
\hline IRDC313.72-3 & $6.5(0.02)$ & $-43.8(0.01)$ & $2.5(0.02)$ & 2.3 & 0.12 \\
\hline IRDC313.72-4 & $4.1(0.02)$ & $-44.0(0.01)$ & $2.0(0.00)$ & 1.9 & 0.12 \\
\hline IRDC316.72-1 & $16.6(0.03)$ & $-38.8(0.01)$ & $4.2(0.02)$ & 3.6 & 0.12 \\
\hline IRDC316.76-1 & $32.7(0.03)$ & $-39.6(0.01)$ & $4.1(0.01)$ & 7.3 & 0.12 \\
\hline IRDC316.72-2 & $9.4(0.03)$ & $-39.3(0.01)$ & $3.6(0.03)$ & 2.4 & 0.12 \\
\hline IRDC316.76-2 & $19.4(0.04)$ & $-40.4(0.01)$ & $4.1(0.02)$ & 4.4 & 0.13 \\
\hline IRDC317.71-1 & $4.3(0.02)$ & $-42.5(0.01)$ & $2.0(0.00)$ & 2.0 & 0.13 \\
\hline IRDC317.71-2 & $6.1(0.02)$ & $-42.7(0.01)$ & $1.8(0.00)$ & 3.1 & 0.12 \\
\hline IRDC317.71-3 & $1.4(0.02)$ & $-43.0(0.03)$ & $1.8(0.00)$ & 0.7 & 0.13 \\
\hline IRDC320.27-1 & $2.4(0.03)$ & $-32.1(0.03)$ & $2.8(0.09)$ & 0.8 & 0.13 \\
\hline IRDC320.27-2 & $0.9(0.02)$ & $-32.5(0.04)$ & $1.7(0.10)$ & 0.4 & 0.13 \\
\hline IRDC320.27-3 & $0.5(0.03)$ & $-32.6(0.07)$ & $1.3(0.24)$ & 0.3 & 0.13 \\
\hline IRDC321.73-1 & $6.6(0.03)$ & $-32.4(0.01)$ & $2.6(0.02)$ & 2.3 & 0.13 \\
\hline IRDC321.73-2 & $5.8(0.02)$ & $-33.1(0.01)$ & $3.0(0.00)$ & 1.8 & 0.13 \\
\hline IRDC321.73-3 & $8.3(0.03)$ & $-31.7(0.01)$ & $3.7(0.03)$ & 2.1 & 0.13 \\
\hline IRDC013.90-1 & $5.4(0.04)$ & $23.1(0.02)$ & $2.4(0.05)$ & 2.0 & 0.12 \\
\hline IRDC013.90-2 & $1.8(0.03)$ & $23.1(0.05)$ & $3.1(0.11)$ & 0.5 & 0.12 \\
\hline IRDC316.45-1 & $4.5(0.03)$ & $-43.5(0.02)$ & $3.3(0.05)$ & 1.2 & 0.15 \\
\hline IRDC316.45-2 & $4.7(0.03)$ & $-43.3(0.02)$ & $3.2(0.05)$ & 1.3 & 0.14 \\
\hline IRDC318.15-1 & $3.7(0.02)$ & $-42.7(0.01)$ & $2.5(0.04)$ & 1.4 & 0.13 \\
\hline IRDC318.15-2 & $3.3(0.02)$ & $-42.0(0.01)$ & $2.1(0.04)$ & 1.4 & 0.13 \\
\hline IRDC309.94-1 & $8.5(0.05)$ & $-59.5(0.02)$ & $4.2(0.05)$ & 1.8 & 0.16 \\
\hline
\end{tabular}

Notes. Columns are name, integrated area obtained from the Gaussian fits, LSR velocity, full linewidth at half maximum, main beam brightness temperature, $1 \sigma$ rms values. 
Table C.5. $\mathrm{HCO}^{+}$line parameters.

\begin{tabular}{|c|c|c|c|c|c|}
\hline Name & $\int_{\left(\mathrm{K} \mathrm{km} \mathrm{s}^{-1}\right)} T_{\mathrm{MB}} \mathrm{d} v$ & $\begin{array}{c}V_{\mathrm{lsr}} \\
\left(\mathrm{km} \mathrm{s}^{-1}\right)\end{array}$ & $\begin{array}{c}\Delta V \\
\left(\mathrm{~km} \mathrm{~s}^{-1}\right)\end{array}$ & $\begin{array}{l}T_{\mathrm{mb}} \\
(\mathrm{K})\end{array}$ & $\begin{array}{c}1 \sigma \mathrm{rms} \\
(\mathrm{K})\end{array}$ \\
\hline IRDC308.13-1 & $4.7(0.04)$ & $-47.6(0.02)$ & $2.7(0.07)$ & 1.5 & 0.17 \\
\hline IRDC308.13-2 & $3.4(0.03)$ & $-47.9(0.02)$ & $2.1(0.05)$ & 1.5 & 0.16 \\
\hline IRDC308.13-3 & $3.0(0.03)$ & $-48.0(0.02)$ & $2.3(0.07)$ & 1.2 & 0.16 \\
\hline IRDC309.13-1 & $4.1(0.07)$ & $-45.0(0.15)$ & $9.1(0.41)$ & 0.4 & 0.16 \\
\hline IRDC309.13-2 & $3.2(0.05)$ & $-46.2(0.09)$ & $5.6(0.20)$ & 0.5 & 0.14 \\
\hline IRDC309.13-3 & $1.6(0.03)$ & $-47.3(0.05)$ & $2.6(0.14)$ & 0.5 & 0.15 \\
\hline IRDC309.37-1 & $13.9(0.14)$ & $-43.4(0.02)$ & $2.8(0.08)$ & 4.5 & 0.31 \\
\hline IRDC309.37-2 & $1.8(0.03)$ & $-43.6(0.05)$ & $2.8(0.13)$ & 0.6 & 0.14 \\
\hline IRDC309.37-3 & $5.9(0.05)$ & $-43.7(0.02)$ & $2.2(0.06)$ & 2.4 & 0.13 \\
\hline IRDC310.39-1 & $8.5(0.05)$ & $-53.0(0.02)$ & $2.3(0.00)$ & 3.4 & 0.12 \\
\hline IRDC310.39-2 & $6.4(0.02)$ & $-52.8(0.01)$ & $2.0(0.00)$ & 3.0 & 0.12 \\
\hline IRDC312.36-1 & $5.7(0.03)$ & $-51.0(0.01)$ & $2.7(0.04)$ & 1.9 & 0.12 \\
\hline IRDC312.36-2 & $1.1(0.04)$ & $-51.4(0.21)$ & $5.7(0.44)$ & 0.1 & 0.12 \\
\hline IRDC313.72-1 & $7.2(0.06)$ & $-44.8(0.02)$ & $2.5(0.00)$ & 2.7 & 0.11 \\
\hline IRDC313.72-2 & $7.1(0.04)$ & $-44.8(0.02)$ & $2.5(0.00)$ & 2.7 & 0.13 \\
\hline IRDC313.72-3 & $8.1(0.04)$ & $-44.2(0.01)$ & $2.6(0.03)$ & 2.8 & 0.12 \\
\hline IRDC313.72-4 & $7.0(0.03)$ & $-44.5(0.01)$ & $2.5(0.00)$ & 2.6 & 0.12 \\
\hline IRDC316.72-1 & $19.6(0.04)$ & $-38.7(0.01)$ & $4.0(0.00)$ & 4.6 & 0.12 \\
\hline IRDC316.76-1 & $30.0(0.04)$ & $-39.0(0.01)$ & $4.0(0.00)$ & 7.0 & 0.12 \\
\hline IRDC316.72-2 & $12.9(0.04)$ & $-38.9(0.01)$ & $5.4(0.05)$ & 2.2 & 0.12 \\
\hline IRDC316.76-2 & $23.2(0.04)$ & $-39.3(0.01)$ & $6.5(0.03)$ & 3.3 & 0.12 \\
\hline IRDC317.71-1 & $4.5(0.02)$ & $-41.9(0.01)$ & $2.1(0.00)$ & 2.0 & 0.13 \\
\hline IRDC317.71-2 & $4.5(0.02)$ & $-42.1(0.01)$ & $2.1(0.00)$ & 2.0 & 0.12 \\
\hline IRDC317.71-3 & $2.3(0.02)$ & $-42.7(0.02)$ & $2.1(0.00)$ & 1.0 & 0.12 \\
\hline IRDC320.27-1 & $1.1(0.03)$ & $-31.0(0.04)$ & $1.5(0.00)$ & 0.7 & 0.12 \\
\hline IRDC320.27-2 & $0.7(0.02)$ & $-33.0(0.03)$ & $1.3(0.10)$ & 0.5 & 0.12 \\
\hline IRDC320.27-3 & $0.4(0.01)$ & $-32.9(0.02)$ & $0.7(0.06)$ & 0.5 & 0.14 \\
\hline IRDC321.73-1 & $9.2(0.03)$ & $-32.7(0.01)$ & $2.4(0.02)$ & 3.4 & 0.13 \\
\hline IRDC321.73-2 & $8.0(0.04)$ & $-32.7(0.02)$ & $5.0(0.06)$ & 1.4 & 0.12 \\
\hline IRDC321.73-3 & $4.3(0.10)$ & $-30.6(0.05)$ & $1.5(0.00)$ & 2.7 & 0.14 \\
\hline IRDC013.90-1 & $5.4(0.03)$ & $23.1(0.01)$ & $2.4(0.04)$ & 2.1 & 0.45 \\
\hline IRDC013.90-2 & $2.1(0.04)$ & $23.5(0.07)$ & $3.4(0.12)$ & 0.5 & 0.20 \\
\hline IRDC316.45-1 & $4.7(0.04)$ & $-43.2(0.03)$ & $4.3(0.09)$ & 1.0 & 0.12 \\
\hline IRDC316.45-2 & $3.6(0.03)$ & $-43.3(0.04)$ & $4.1(0.09)$ & 0.8 & 0.13 \\
\hline IRDC318.15-1 & $1.9(0.02)$ & $-43.3(0.02)$ & $1.8(0.00)$ & 0.9 & 0.12 \\
\hline IRDC318.15-2 & $0.9(0.02)$ & $-43.2(0.06)$ & $1.8(0.00)$ & 0.5 & 0.12 \\
\hline IRDC309.94-1 & $9.9(0.06)$ & $-60.3(0.03)$ & $5.4(0.08)$ & 1.7 & 0.15 \\
\hline
\end{tabular}

Notes. Columns are name, integrated area obtained from the Gaussian fits, LSR velocity, full linewidth at half maximum, main beam brightness temperature, $1 \sigma$ rms values.

Table C.6. HNCO line parameters.

\begin{tabular}{lccccc}
\hline \hline Name & $\begin{array}{c}\int T_{\mathrm{MB}} \mathrm{d} v \\
\left(\mathrm{~K} \mathrm{~km} \mathrm{~s}^{-1}\right)\end{array}$ & $\begin{array}{c}V_{\mathrm{lsr}} \\
\left(\mathrm{km} \mathrm{s}^{-1}\right)\end{array}$ & $\begin{array}{c}\Delta V \\
\left(\mathrm{~km} \mathrm{~s}^{-1}\right)\end{array}$ & $\begin{array}{c}T_{\mathrm{mb}} \\
(\mathrm{K})\end{array}$ & $\begin{array}{c}1 \sigma \mathrm{rm} \\
(\mathrm{K})\end{array}$ \\
\hline IRDC309.37-1 & $1.3(0.07)$ & $-42.4(0.12)$ & $2.3(0.36)$ & 0.5 & 0.15 \\
IRDC310.39-1 & $0.3(0.02)$ & $-53.8(0.08)$ & $1.3(0.20)$ & 0.2 & 0.07 \\
IRDC312.36-1 & $0.4(0.02)$ & $-50.4(0.14)$ & $2.5(0.26)$ & 0.1 & 0.06 \\
IRDC313.72-1 & $0.5(0.02)$ & $-44.3(0.06)$ & $1.4(0.19)$ & 0.3 & 0.06 \\
IRDC313.72-2 & $0.6(0.02)$ & $-44.1(0.13)$ & $2.9(0.30)$ & 0.1 & 0.05 \\
IRDC313.72-3 & $0.3(0.02)$ & $-43.6(0.11)$ & $2.0(0.36)$ & 0.1 & 0.06 \\
IRDC316.72-1 & $0.9(0.03)$ & $-39.1(0.08)$ & $2.6(0.18)$ & 0.3 & 0.06 \\
IRDC316.76-1 & $1.0(0.03)$ & $-39.7(0.10)$ & $3.3(0.29)$ & 0.2 & 0.06 \\
IRDC317.71-1 & $0.6(0.03)$ & $-43.5(0.09)$ & $2.0(0.23)$ & 0.3 & 0.07 \\
IRDC317.71-2 & $1.1(0.04)$ & $-43.7(0.10)$ & $3.4(0.31)$ & 0.3 & 0.06 \\
IRDC320.27-1 & $0.3(0.01)$ & $-31.7(0.07)$ & $1.3(0.17)$ & 0.2 & 0.06 \\
IRDC316.45-1 & $0.3(0.02)$ & $-43.4(0.08)$ & $1.4(0.19)$ & 0.2 & 0.07 \\
IRDC316.45-2 & $0.4(0.02)$ & $-43.2(0.07)$ & $1.5(0.16)$ & 0.3 & 0.07 \\
IRDC309.94-1 & $1.1(0.03)$ & $-59.4(0.08)$ & $2.6(0.22)$ & 0.3 & 0.07 \\
\hline
\end{tabular}

Notes. Columns are name, integrated area obtained from the Gaussian fits, LSR velocity, full linewidth at half maximum, main beam brightness temperature, $1 \sigma$ rms values. 
Table C.7. SiO line parameters.

\begin{tabular}{lccccc}
\hline \hline Name & $\begin{array}{c}\int T_{\mathrm{MB}} \mathrm{d} v \\
\left(\mathrm{~K} \mathrm{~km} \mathrm{~s}^{-1}\right)\end{array}$ & $\begin{array}{c}V_{\mathrm{lsr}} \\
\left(\mathrm{km} \mathrm{s}^{-1}\right)\end{array}$ & $\begin{array}{c}\Delta V \\
\left(\mathrm{~km} \mathrm{~s}^{-1}\right)\end{array}$ & $\begin{array}{c}T_{\mathrm{mb}} \\
(\mathrm{K})\end{array}$ & $\begin{array}{c}1 \sigma \mathrm{rms} \\
(\mathrm{K})\end{array}$ \\
\hline IRDC309.37-1 & $0.9(0.08)$ & $-42.6(0.2)$ & $2.8(0.5)$ & 0.3 & 0.18 \\
IRDC310.39-1 & $0.6(0.03)$ & $-53.3(0.2)$ & $3.9(0.4)$ & 0.1 & 0.08 \\
IRDC312.36-1 & $0.2(0.03)$ & $-50.8(0.2)$ & $2.0(0.5)$ & 0.1 & 0.08 \\
IRDC313.72-1 & $1.6(0.04)$ & $-43.9(0.1)$ & $4.5(0.3)$ & 0.3 & 0.08 \\
IRDC313.72-2 & $1.7(0.05)$ & $-44.4(0.1)$ & $5.1(0.4)$ & 0.3 & 0.08 \\
IRDC313.72-3 & $1.3(0.06)$ & $-41.9(0.3)$ & $8.2(0.8)$ & 0.1 & 0.08 \\
IRDC313.72-4 & $0.5(0.03)$ & $-44.1(0.2)$ & $3.4(0.4)$ & 0.1 & 0.08 \\
IRDC316.76-1 & $2.4(0.07)$ & $-39.5(0.2)$ & $8.9(0.7)$ & 0.2 & 0.08 \\
IRDC317.71-3 & $0.3(0.03)$ & $-42.1(0.2)$ & $1.9(0.5)$ & 0.1 & 0.08 \\
IRDC321.73-1 & $2.1(0.05)$ & $-32.1(0.1)$ & $4.8(0.3)$ & 0.4 & 0.08 \\
IRDC309.94-1 & $2.4(0.05)$ & $-59.6(0.2)$ & $5.0(0.0)$ & 0.4 & 0.10 \\
\hline
\end{tabular}

Notes. Columns are name, integrated area obtained from the Gaussian fits, LSR velocity, full linewidth at half maximum, main beam brightness temperature, $1 \sigma \mathrm{rms}$ values.

Table C.8. $\mathrm{H}^{13} \mathrm{CO}^{+}$line parameters.

\begin{tabular}{|c|c|c|c|c|c|c|c|}
\hline Name & $\underset{\left(\mathrm{K} \mathrm{km} \mathrm{s}^{-1}\right)}{\int T_{\mathrm{MB}} \mathrm{d} v}$ & $\begin{array}{c}V_{\mathrm{lsr}} \\
\left(\mathrm{km} \mathrm{s}^{-1}\right)\end{array}$ & $\begin{array}{c}\Delta V \\
\left(\mathrm{~km} \mathrm{~s}^{-1}\right)\end{array}$ & $\begin{array}{l}T_{\mathrm{mb}} \\
(\mathrm{K})\end{array}$ & $\tau$ & $\begin{array}{c}1 \sigma \mathrm{rms} \\
(\mathrm{K})\end{array}$ & $\delta V$ \\
\hline IRDC308.13-1 & $0.6(0.06)$ & $-46.7(0.28)$ & $2.5(0.41)$ & 0.2 & 0.07 & 0.08 & $-0.8(0.12)$ \\
\hline IRDC309.13-1 & $0.8(0.05)$ & $-44.7(0.14)$ & $2.1(0.31)$ & 0.3 & 0.20 & 0.07 & $-0.2(0.09)$ \\
\hline IRDC309.13-3 & $0.5(0.05)$ & $-45.2(0.12)$ & $1.5(0.39)$ & 0.3 & 0.17 & 0.08 & $-2.1(0.35)$ \\
\hline IRDC309.37-1 & $2.6(0.13)$ & $-42.6(0.09)$ & $2.1(0.26)$ & 1.1 & 0.20 & 0.16 & $-0.8(0.06)$ \\
\hline IRDC309.37-3 & $1.3(0.06)$ & $-42.4(0.15)$ & $3.2(0.34)$ & 0.3 & 0.10 & 0.08 & $-1.2(0.06)$ \\
\hline IRDC310.39-1 & $1.9(0.04)$ & $-52.4(0.04)$ & $1.9(0.11)$ & 0.9 & 0.19 & 0.08 & $-0.6(0.03)$ \\
\hline IRDC310.39-2 & $1.2(0.05)$ & $-52.3(0.09)$ & $2.2(0.20)$ & 0.5 & 0.12 & 0.08 & $-0.4(0.04)$ \\
\hline IRDC312.36-1 & $1.3(0.06)$ & $-50.6(0.13)$ & $3.0(0.35)$ & 0.4 & 0.12 & 0.08 & $-0.3(0.04)$ \\
\hline IRDC312.36-2 & $0.2(0.03)$ & $-48.8(0.20)$ & $1.4(0.38)$ & 0.1 & 0.11 & 0.08 & $-2.5(0.49)$ \\
\hline IRDC313.72-1 & $1.1(0.08)$ & $-43.9(0.19)$ & $2.5(0.63)$ & 0.4 & 0.10 & 0.08 & $-0.8(0.11)$ \\
\hline IRDC313.72-2 & $1.1(0.05)$ & $-43.9(0.10)$ & $2.2(0.31)$ & 0.4 & 0.11 & 0.08 & $-0.8(0.06)$ \\
\hline IRDC313.72-3 & $0.7(0.04)$ & $-43.5(0.10)$ & $1.8(0.21)$ & 0.4 & 0.09 & 0.08 & $-0.7(0.07)$ \\
\hline IRDC313.72-4 & $0.8(0.05)$ & $-43.3(0.12)$ & $2.2(0.30)$ & 0.3 & 0.09 & 0.08 & $-1.2(0.09)$ \\
\hline IRDC316.72-1 & $4.4(0.06)$ & $-38.8(0.04)$ & $3.1(0.10)$ & 1.2 & 0.22 & 0.08 & $0.1(0.01)$ \\
\hline IRDC316.76-1 & $5.2(0.06)$ & $-39.7(0.03)$ & $3.2(0.09)$ & 1.5 & 0.18 & 0.08 & $0.7(0.01)$ \\
\hline IRDC316.72-2 & $2.7(0.05)$ & $-39.5(0.04)$ & $2.2(0.09)$ & 1.1 & 0.33 & 0.08 & $0.6(0.02)$ \\
\hline IRDC316.76-2 & $3.1(0.05)$ & $-40.5(0.04)$ & $2.4(0.10)$ & 1.1 & 0.26 & 0.08 & $1.1(0.02)$ \\
\hline IRDC317.71-1 & $1.3(0.04)$ & $-43.3(0.07)$ & $1.9(0.17)$ & 0.6 & 0.18 & 0.08 & $1.3(0.07)$ \\
\hline IRDC317.71-2 & $1.7(0.05)$ & $-43.8(0.05)$ & $1.9(0.16)$ & 0.8 & 0.23 & 0.08 & $1.7(0.07)$ \\
\hline IRDC317.71-3 & $0.4(0.04)$ & $-43.7(0.20)$ & $1.7(0.47)$ & 0.2 & 0.08 & 0.09 & $1.0(0.19)$ \\
\hline IRDC320.27-1 & $0.7(0.03)$ & $-31.9(0.05)$ & $1.1(0.12)$ & 0.5 & 0.28 & 0.09 & $0.8(0.10)$ \\
\hline IRDC321.73-1 & $1.6(0.04)$ & $-32.1(0.04)$ & $1.5(0.09)$ & 0.9 & 0.20 & 0.08 & $-0.5(0.03)$ \\
\hline IRDC321.73-2 & $0.6(0.04)$ & $-33.4(0.08)$ & $1.3(0.22)$ & 0.4 & 0.16 & 0.08 & $0.7(0.10)$ \\
\hline IRDC321.73-3 & $1.2(0.06)$ & $-31.6(0.14)$ & $2.5(0.31)$ & 0.4 & 0.10 & 0.09 & $1.0(0.07)$ \\
\hline IRDC013.90-1 & $1.0(0.04)$ & $23.3(0.09)$ & $1.9(0.18)$ & 0.5 & 0.14 & 0.08 & $-0.2(0.04)$ \\
\hline IRDC013.90-2 & $0.3(0.02)$ & $22.3(0.03)$ & $0.4(0.10)$ & 0.6 & 0.35 & 0.08 & $1.2(0.68)$ \\
\hline IRDC316.45-1 & $0.8(0.05)$ & $-43.5(0.12)$ & $1.9(0.25)$ & 0.4 & 0.16 & 0.08 & $0.2(0.06)$ \\
\hline IRDC316.45-2 & $1.1(0.05)$ & $-43.3(0.10)$ & $2.2(0.23)$ & 0.4 & 0.21 & 0.08 & $-0.0(0.05)$ \\
\hline IRDC318.15-1 & $0.7(0.04)$ & $-42.5(0.09)$ & $1.6(0.21)$ & 0.4 & 0.17 & 0.08 & $-0.7(0.08)$ \\
\hline IRDC318.15-2 & $0.6(0.04)$ & $-42.0(0.10)$ & $1.5(0.20)$ & 0.3 & 0.19 & 0.08 & $-1.1(0.13)$ \\
\hline IRDC309.94-1 & $1.7(0.06)$ & $-59.3(0.07)$ & $2.1(0.17)$ & 0.7 & 0.25 & 0.11 & $-0.9(0.05)$ \\
\hline
\end{tabular}

Notes. Columns are name, integrated area obtained from the Gaussian fits, LSR velocity, full linewidth at half maximum, main beam brightness temperature, optical depth, $1 \sigma \mathrm{rms}$ values, $\delta V=\left(V_{\mathrm{HCO}^{+}}-V_{\mathrm{H}^{13} \mathrm{CO}^{+}}\right) / \Delta V_{\mathrm{H}^{13} \mathrm{CO}^{+}}$. 
A\&A 527, A88 (2011)

Table C.9. Integrated areas, measured by summing the channels between suitable velocity limits under the line.

\begin{tabular}{|c|c|c|c|c|c|c|c|c|c|c|c|}
\hline Name & $\begin{array}{c}\mathrm{N}_{2} \mathrm{H}^{+} \\
\left(\mathrm{K} \mathrm{km} \mathrm{s}^{-1}\right)\end{array}$ & $\begin{array}{c}{ }^{13} \mathrm{CS} \\
\left(\mathrm{K} \mathrm{km} \mathrm{s}^{-1}\right)\end{array}$ & $\begin{array}{c}\mathrm{HC}_{3} \mathrm{~N} \\
\left(\mathrm{~K} \mathrm{~km} \mathrm{~s}^{-1}\right)\end{array}$ & $\begin{array}{c}\mathrm{HNC} \\
\left(\mathrm{K} \mathrm{km} \mathrm{s}^{-1}\right)\end{array}$ & $\begin{array}{c}\mathrm{HCO}^{+} \\
\left(\mathrm{K} \mathrm{km} \mathrm{s}^{-1}\right)\end{array}$ & $\begin{array}{c}\mathrm{HCN} \\
\left(\mathrm{K} \mathrm{km} \mathrm{s}^{-1}\right)\end{array}$ & $\begin{array}{c}\mathrm{HNCO} \\
\left(\mathrm{K} \mathrm{km} \mathrm{s}^{-1}\right)\end{array}$ & $\begin{array}{c}\mathrm{C}_{2} \mathrm{H} \\
\left(\mathrm{K} \mathrm{km} \mathrm{s}^{-1}\right)\end{array}$ & $\begin{array}{c}\mathrm{SiO} \\
\left(\mathrm{K} \mathrm{km} \mathrm{s}^{-1}\right)\end{array}$ & $\begin{array}{c}\mathrm{H}^{13} \mathrm{CN} \\
\left(\mathrm{K} \mathrm{km} \mathrm{s}^{-1}\right)\end{array}$ & $\begin{array}{c}\mathrm{CH}_{3} \mathrm{C}_{2} \mathrm{H} \\
\left(\mathrm{K} \mathrm{km} \mathrm{s}^{-1}\right)\end{array}$ \\
\hline IRDC308.13-1 & 4.2 & & & 4.8 & 6.2 & 5.3 & & & & & \\
\hline IRDC308.13-2 & 2.1 & & & 2.6 & 4.2 & 2.9 & & 0.6 & & & \\
\hline IRDC308.13-3 & 1.6 & & & 2.9 & 3.7 & 3.3 & & & & & \\
\hline IRDC309.13-1 & 5.0 & & & 3.1 & 4.1 & 4.5 & & 0.4 & & & \\
\hline IRDC309.13-2 & 2.8 & & & 2.5 & 3.2 & 3.9 & & & & & \\
\hline IRDC309.13-3 & 1.5 & & & 0.8 & 1.9 & 2.3 & & & & & \\
\hline IRDC309.37-1 & 29.1 & & 1.6 & 13.6 & 17.9 & 15.4 & 1.4 & 3.2 & & & \\
\hline IRDC309.37-2 & & & & 1.2 & 2.4 & 2.4 & & & & & \\
\hline IRDC309.37-3 & 4.6 & & & 4.8 & 6.8 & 6.0 & & & & & \\
\hline IRDC310.39-1 & 13.4 & & 1.9 & 8.1 & 13.3 & 12.5 & 0.2 & 1.7 & 0.4 & & \\
\hline IRDC310.39-2 & 5.4 & & 1.2 & 5.5 & 8.7 & 9.0 & & 1.4 & & & \\
\hline IRDC312.36-1 & 6.2 & & 0.2 & 4.9 & 6.6 & 6.6 & 0.6 & 0.7 & & & \\
\hline IRDC312.36-2 & 1.3 & & & 1.9 & 1.0 & 2.1 & & & & & \\
\hline IRDC313.72-1 & 8.0 & & 1.7 & 6.9 & 11.3 & 7.8 & 0.6 & 1.1 & 1.6 & & \\
\hline IRDC313.72-2 & 8.6 & 0.3 & 1.2 & 6.7 & 10.6 & 8.0 & 0.5 & 0.9 & 1.9 & & \\
\hline IRDC $313.72-3$ & 7.1 & & 0.6 & 7.3 & 9.1 & 7.3 & 0.4 & 0.6 & 1.3 & & \\
\hline IRDC313.72-4 & 5.9 & & & 5.7 & 9.3 & 5.9 & & & 0.6 & & \\
\hline IRDC316.72-1 & 18.4 & & 1.1 & 17.1 & 23.2 & 18.6 & 0.8 & 2.8 & & 1.9 & 0.3 \\
\hline IRDC316.76-1 & 50.7 & 0.9 & 4.9 & 34.1 & 34.6 & 33.8 & 1.1 & 7.1 & 2.3 & 4.8 & 0.7 \\
\hline IRDC316.72-2 & 11.8 & & 0.8 & 10.2 & 14.1 & 10.9 & & 1.9 & & & 0.3 \\
\hline IRDC316.76-2 & 27.4 & 0.5 & 2.9 & 21.4 & 23.3 & 27.4 & & 7.1 & & 3.3 & 0.3 \\
\hline IRDC317.71-1 & 10.5 & & 0.4 & 5.0 & 5.6 & 2.7 & 0.7 & 0.8 & & & \\
\hline IRDC317.71-2 & 21.9 & & 2.9 & 8.1 & 5.6 & 4.9 & 1.3 & 1.5 & & 1.5 & 0.57 \\
\hline IRDC317.71-3 & 3.5 & & & 2.3 & 2.5 & 1.8 & & & 1.0 & & \\
\hline IRDC320.27-1 & 2.5 & & & 2.6 & 2.6 & 3.8 & & & & & \\
\hline IRDC320.27-2 & 1.7 & & & 1.1 & 0.7 & 1.7 & & & & & \\
\hline IRDC320.27-3 & 0.6 & & & 0.8 & 0.3 & 0.8 & & & & & \\
\hline IRDC321.73-1 & 11.0 & & 1.3 & 7.0 & 10.5 & 11.1 & & 1.4 & 2.5 & & \\
\hline IRDC321.73-2 & 5.5 & & 1.7 & 6.9 & 8.4 & 7.9 & & 0.9 & & & \\
\hline IRDC321.73-3 & 7.5 & & 1.3 & 8.4 & 10.4 & 8.9 & & 0.8 & & & \\
\hline IRDC013.90-1 & 5.6 & & 0.7 & 5.6 & 6.3 & 5.4 & & 0.9 & & & \\
\hline IRDC013.90-2 & 2.4 & & & 1.4 & 0.8 & 1.9 & & & & & \\
\hline IRDC316.45-1 & 7.0 & & 1.0 & 4.7 & 5.1 & 4.0 & 0.1 & 0.7 & & & \\
\hline IRDC316.45-2 & 6.2 & & & 4.9 & 3.4 & 4.0 & 0.4 & 0.7 & & & \\
\hline IRDC318.15-1 & 3.7 & & & 3.9 & 3.0 & 3.3 & & 0.7 & & & \\
\hline IRDC318.15-2 & 4.6 & & & 3.9 & 1.8 & 2.9 & & 0.5 & & & \\
\hline IRDC309.94-1 & 22.0 & & 3.7 & 9.4 & 11.0 & 11.8 & 0.9 & 2.4 & & 2.1 & \\
\hline
\end{tabular}

Notes. Columns are name, integrated area for every species. 